OPEN ACCESS

Edited by:

Claudia Torino,

Institute of Clinical Physiology, Italian

National Research Council, Italy

Reviewed by:

Heon Yung Gee,

Yonsei University, South Korea

Moe R. Mahjoub,

Washington University in St. Louis,

United States

Antonio Demetrio Vilasi,

National Research Council (CNR), Italy

${ }^{*}$ Correspondence:

Alexandre Benmerah

Alexandre.benmerah@inserm.fr

Marijn F. Stokman

M.F.Stokman@umcutrecht.ni

Specialty section

This article was submitted to

Molecular Medicine,

a section of the journal

Frontiers in Cell and Developmental

Biology

Received: 13 January 2021

Accepted: 19 April 2021

Published: 13 May 2021

Citation:

Stokman MF, Saunier S and

Benmerah A (2021) Renal Ciliopathies: Sorting Out Therapeutic

Approaches for Nephronophthisis.

Front. Cell Dev. Biol. 9:653138.

doi: 10.3389/fcell.2021.653138

\section{Renal Ciliopathies: Sorting Out Therapeutic Approaches for Nephronophthisis}

\author{
Marijn F. Stokman ${ }^{1,2 *}$, Sophie Saunier ${ }^{2}$ and Alexandre Benmerah ${ }^{2 *}$ \\ ${ }^{1}$ Department of Genetics, University Medical Center Utrecht, Utrecht, Netherlands, ${ }^{2}$ Université de Paris, Imagine Institute, \\ Laboratory of Inherited Kidney Diseases, INSERM UMR 1163, Paris, France
}

Nephronophthisis (NPH) is an autosomal recessive ciliopathy and a major cause of end-stage renal disease in children. The main forms, juvenile and adult NPH, are characterized by tubulointerstitial fibrosis whereas the infantile form is more severe and characterized by cysts. NPH is caused by mutations in over 20 different genes, most of which encode components of the primary cilium, an organelle in which important cellular signaling pathways converge. Ciliary signal transduction plays a critical role in kidney development and tissue homeostasis, and disruption of ciliary signaling has been associated with cyst formation, epithelial cell dedifferentiation and kidney function decline. Drugs have been identified that target specific signaling pathways (for example cAMP/PKA, Hedgehog, and mTOR pathways) and rescue NPH phenotypes in in vitro and/or in vivo models. Despite identification of numerous candidate drugs in rodent models, there has been a lack of clinical trials and there is currently no therapy that halts disease progression in NPH patients. This review covers the most important findings of therapeutic approaches in NPH model systems to date, including hypothesis-driven therapies and untargeted drug screens, approached from the pathophysiology of NPH. Importantly, most animal models used in these studies represent the cystic infantile form of $\mathrm{NPH}$, which is less prevalent than the juvenile form. It appears therefore important to develop new models relevant for juvenile/adult NPH. Alternative non-orthologous animal models and developments in patient-based in vitro model systems are discussed, as well as future directions in personalized therapy for $\mathrm{NPH}$.

Keywords: hereditary kidney disease, ciliopathy, nephronophthisis, signaling, cell cycle, drug screen, therapy, gene therapy

\section{INTRODUCTION}

Nephronophthisis (NPH) is an autosomal recessive kidney disease that accounts for up to $15 \%$ of end-stage renal disease (ESRD) in children (Hamiwka et al., 2008; Salomon et al., 2009). In addition, homozygous deletions in the NPHP1 gene, the most frequent cause of $\mathrm{NPH}(\approx 50 \%$ of genetically diagnosed cases), have recently been shown to be an important cause of renal disease in adults (Snoek et al., 2018). 
Three clinical subtypes of NPH can be discerned. In juvenile NPH, the most prevalent form, ESRD develops at a median age of 13 years (Hildebrandt et al., 2009). Juvenile $\mathrm{NPH}$ is characterized by small to normal-sized kidneys that show marked tubulointerstitial fibrosis, thickened and disrupted tubular basement membranes and, in less than $50 \%$ of patients, corticomedullary cysts that arise from distal tubules in advanced stages of the disease (Hildebrandt et al., 2009). Patients present with a urine concentration defect leading to polyuria and polydipsia, anemia, proteinuria (in advanced stages) and progressive renal insufficiency (König et al., 2017; Stokman et al., 2018). The adult form of NPH is clinically and histologically similar to juvenile NPH and leads to adult-onset ESRD ( $>15$ years old). This form is difficult to distinguish clinically from autosomal dominant tubulointerstitial kidney disease (ADTKD) which is also characterized by tubular damage, interstitial fibrosis and corticomedullary microcysts (Devuyst et al., 2019). In contrast, autosomal dominant polycystic kidney disease (ADPKD), which is more prevalent, is typically adult-onset with slow progression to ESRD [onset from 58 to 80 years (Chebib and Torres, 2016)] and characterized by enlarged kidneys with cysts distributed throughout the renal parenchyma (Harris and Torres, 2002).

In contrast to juvenile and adult NPH, infantile NPH leads to ESRD before the age of 5 years and typically presents with enlarged cystic kidneys, severe hypertension and a rapid disease course, making it difficult to distinguish from autosomal recessive polycystic kidney disease (ARPKD) based on renal symptoms only (Gagnadoux et al., 1989; Otto et al., 2003; Waters and Beales, 2011; Bergmann, 2018). Infantile NPH is characterized histologically by cortical cysts, absence of tubular basement membrane thickening and only moderate fibrosis (Gagnadoux et al., 1989; Salomon et al., 2009; Tory et al., 2009). This raises the question whether infantile NPH is really part of the NPH spectrum or rather represents a distinct subtype of cystic kidney disease.

Mutations in more than 20 different genes have been identified to cause NPH (Braun and Hildebrandt, 2017; Devlin and Sayer, 2019). Most of these genes encode proteins that localize to primary cilia and their disruption causes defects in cilia formation, length and/or composition. NPH therefore falls into the category of ciliopathies, a group of multisystemic disorders linked to primary cilia dysfunction. Primary (non-motile or sensory) cilia (PC) are microtubulebased antennae present on the surface of nearly all cell types in vertebrates during development and/or in adult tissues, from where they sense mechanical stress (shear stress), molecules (odor, light) and control key signaling pathways (Hedgehog, Wnt, TGF $\beta$, etc.). PC dysfunction is therefore associated with a variety of phenotypes in different organs including kidney failure, retinal dystrophy, liver fibrosis, skeletal dysplasia, cerebellar vermis hypoplasia and obesity. In the kidney, PC are present on the apical surface of most tubular cells from where they integrate different stimuli during kidney homeostasis and function. The discovery of the ciliary localization and functions of polycystin-1 and polycystin-2, the products of the ADPKD genes PKD1 and PKD2, led to the hypothesis that cilia control oriented cell division and proliferation of tubular cells, and that ciliary defects therefore result in cystic kidneys, which became a classic sign of ciliopathies.

In accordance with the role of PC in most organ systems, $10-20 \%$ of NPH patients have extrarenal manifestations defining syndromic forms, including Senior-Løken syndrome (NPH and retinal dystrophy), Bardet-Biedl syndrome (retinal dystrophy, obesity and polydactyly), Joubert syndrome (cerebellar vermis hypoplasia, hypotonia and developmental delay) and cranioectodermal dysplasia (narrow thorax and short stature) (Braun and Hildebrandt, 2017).

Current drug therapy for NPH is merely supportive, aimed at treatment of anemia, hypertension, growth retardation and other symptoms related to chronic kidney disease (CKD) (Braun and Hildebrandt, 2017). Although symptomatic treatment can prevent accelerated decline of renal function (for example due to hypertension) and improve quality of life for NPH patients, it does not target underlying disease mechanisms and ESRD cannot be prevented. The only curative treatment for NPH patients is a renal transplantation, as NPH does not recur in the transplanted kidney (Hamiwka et al., 2008; Tayfur et al., 2011).

Therapy for NPH-related ciliopathies has been an active field of research for more than 15 years. Next-generation sequencing techniques have accelerated gene discovery and uncovered numerous targetable genes and pathways. The most important drug targets for renal ciliopathies (i.e., NPH and PKD) have been reviewed by Molinari and Sayer (2017). Most of these drugs target mechanisms discovered in the context of ADPKD and were subsequently shown to also reduce cystogenesis in mouse or zebrafish models of (mostly infantile) NPH. Contrary to ADPKD, however, tubulointerstitial fibrosis is more important than cystogenesis in the pathophysiology of juvenile NPH. Consequently, aberrant DNA damage-response signaling and proinflammatory signaling, which have been proposed to contribute to fibrosis in juvenile NPH (Slaats et al., 2016), are interesting emerging targets and readouts. However, underlying disease mechanisms and potential targets for therapeutic interventions are not fully elucidated. Moreover, in contrast to ADPKD, thus far no clinical trial has been conducted in NPH patients.

If juvenile NPH is detected in an early disease stage, there is a therapeutic window of a few years in which treatment could delay or prevent renal replacement therapy. Previous reviews of therapies for renal ciliopathies focused on models of PKD and cystic (infantile) NPH or did not discern between renal and extrarenal manifestations. In this review we summarize the results from two main strategies for therapeutic intervention in NPH specifically that have been investigated in in vitro and in vivo model systems: pharmacotherapy (hypothesis-driven approaches including repurposed medication from PKD and untargeted drug screens) and gene-based therapy, in relation to the pathophysiology of different aspects of the NPH phenotype. Promising developments in personalized disease modeling and drug delivery are discussed, as well as challenges in clinical trials for NPH and orphan-drug 

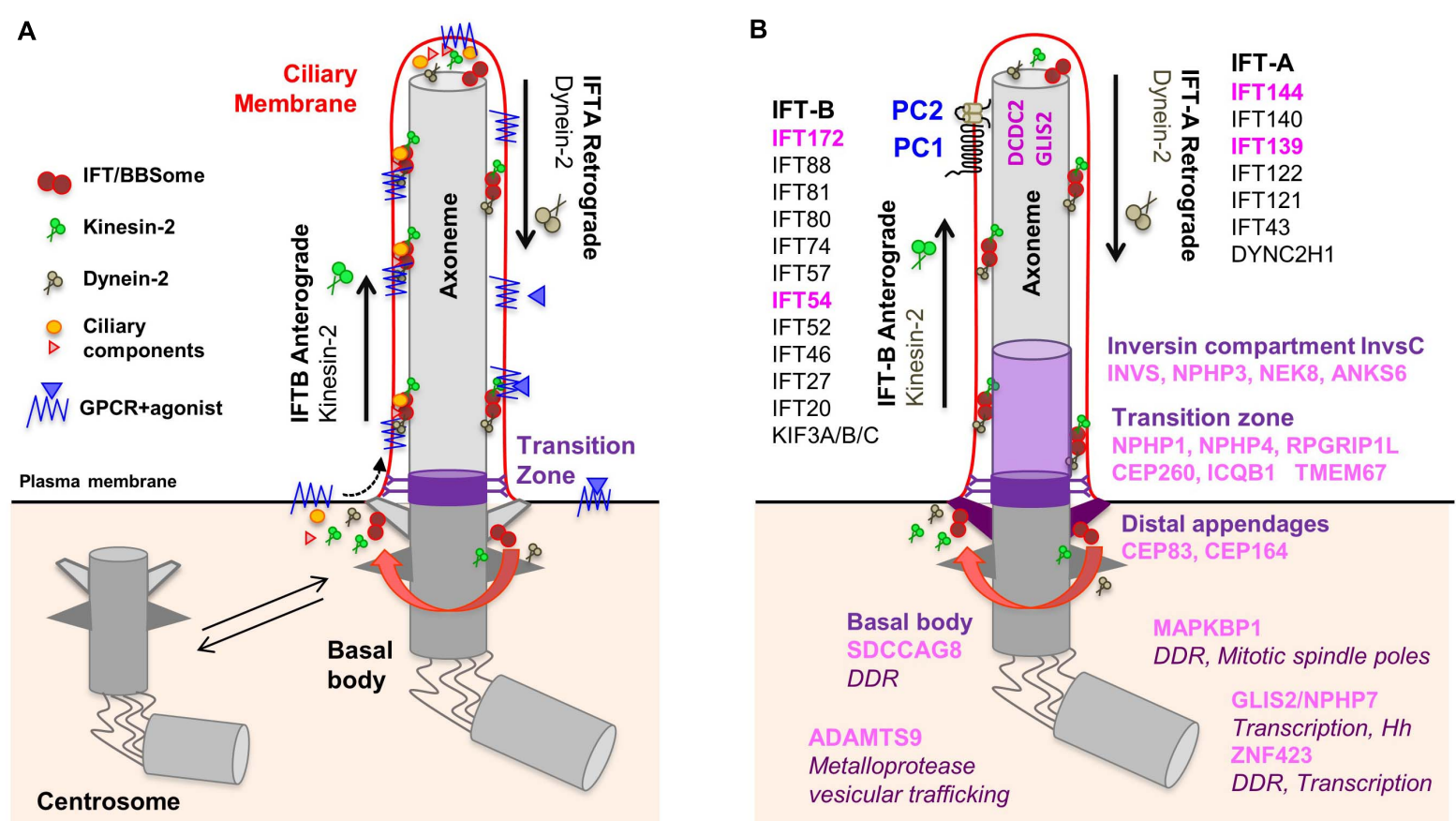

FIGURE 1 | Composition of the primary cilium. (A) The mother centriole docks to the apical plasma membrane and forms the basal body which is connected to the plasma membrane by transition fibers. The basal body anchors the ciliary axoneme, consisting of 9+0 microtubule doublets. At the transition zone, Y-linkers connect the axoneme to the ciliary membrane. The transition zone functions as a gate through which membrane receptors and other proteins can enter the cilium. Intraflagellar transport (IFT) inside the cilium is mediated by kinesin-2 motors (anterograde transport) and dynein motors (retrograde transport). (B) NPH proteins localize to distinct functional compartments of the primary cilium. IFT proteins in pink are encoded by NPH-associated genes. No ciliary localization was demonstrated for ZNF423 and MAPKBP1.

development. Finally, we provide a rationale for future studies in this field.

\section{PATHOPHYSIOLOGY OF NEPHRONOPHTHISIS}

\section{Genetic Basis}

Most ciliopathy-associated genes encode proteins that localize to PC which are assembled in quiescent cells $\left(G_{0}\right)$ from the centrosome, called 'basal body' when engaged in ciliogenesis (Figure 1A). During this process, the mother centriole docks onto cellular membranes through its distal appendages and then the distal ends of the nine centriolar microtubule doublets extend to form the ciliary axoneme which is surrounded by the ciliary membrane. The generation and maintenance of the unique molecular composition of the ciliary compartment (proteins and lipids) is based on the presence of the transition zone which acts as a gate at the base of the cilium and a highly conserved active transport machinery, the intraflagellar transport (IFT), which mediates in and out trafficking across this barrier through anterograde (IFT-B) and retrograde (IFT-A) transport along axonemal microtubules. The IFT and the BBSome, a protein complex encoded by genes mutated in Bardet-Biedl Syndrome, are also involved in the trafficking of signaling receptors enriched at the ciliary membrane such as G-protein coupled receptors (GPCRs) (Reiter et al., 2017).

Nephronophthisis is caused by mutations in 22 genes (full list in Table 1), which encode NPH proteins (NPHP). Of note, not all these genes have an official NPHP alias. Most NPHPs localize to and define three functionally important ciliary subdomains: the transition zone (NPHP1, NPHP4, IQCB1/NPHP5, CEP290/NPHP6 and RPGRIP1L/NPHP8), basal body/centrosome (SDCCAG8/NPHP10, CEP164/NPHP14, CEP83/NPHP18) and inversin compartment (Figure 1B) (Braun and Hildebrandt, 2017; Reiter et al., 2017; Devlin and Sayer, 2019). The inversin compartment (InvsC) comprises the products of four genes, INVS/NPHP2, NPHP3, NEK8/NPHP9 and $A N K S 6 / N P H P 16$, all of which have been implicated in infantile-onset $\mathrm{NPH}$ in addition to juvenile and/or adult $\mathrm{NPH}$ depending on the severity of the mutations (Gagnadoux et al., 1989; Olbrich et al., 2003; Otto et al., 2003, 2008; Bergmann et al., 2008; Tory et al., 2009; Hoff et al., 2013; Rajagopalan et al., 2015; Grampa et al., 2016). In addition to the above ciliary subdomains, NPH-causing mutations were also identified in genes encoding subunits of the IFT-A (TTC21B/IFT139/NPHP12, WDR19/IFT144/NPHP13) and IFT-B (IFT172/NPHP17, TRAF3IP1/IFT54/SLS9) sub-complexes (Bredrup et al., 2011; Davis et al., 2011; Halbritter et al., 2013a; Bizet et al., 2015). Importantly, NPH-related renal involvement was also reported in individuals harboring mutations in 
TABLE 1 | Localization of NPH proteins and associated phenotypes.

\begin{tabular}{|c|c|c|c|c|c|c|}
\hline $\begin{array}{l}\text { Ciliary localization } \\
\text { Function }\end{array}$ & Gene & Locus & $\begin{array}{l}\text { Extraciliary } \\
\text { localization/function }\end{array}$ & $\begin{array}{l}\text { NPH } \\
\text { subtype(s) }\end{array}$ & $\begin{array}{l}\text { Extrarenal features (Halbritter et al., 2013b; } \\
\text { Braun and Hildebrandt, 2017; König et al., } \\
\text { 2017; Luo and Tao, 2018) }\end{array}$ & References \\
\hline \multirow[t]{6}{*}{ Transition zone (TZ) } & NPHP1 & NPHP1 & Cell junctions & Juvenile, adult & $\begin{array}{l}\text { Retinitis pigmentosa, neurologic symptoms, liver } \\
\text { disease }\end{array}$ & $\begin{array}{l}\text { Hildebrandt et al. (1997); Saunier et al. } \\
\text { (1997) }\end{array}$ \\
\hline & NPHP4 & NPHP4 & $\begin{array}{l}\text { Cell junctions Hippo } \\
\text { signaling }\end{array}$ & Juvenile, adult & $\begin{array}{l}\text { Retinitis pigmentosa, neurologic symptoms, } \\
\text { congenital heart disease, liver disease (all } \\
\text { infrequent) }\end{array}$ & Mollet et al. (2002); Otto et al. (2002) \\
\hline & IQCB1 & NPHP5 & - & Juvenile, adult & $\begin{array}{l}\text { Retinitis pigmentosa (in all patients), leber } \\
\text { congenital amaurosis, neurologic symptoms }\end{array}$ & $\begin{array}{l}\text { Otto et al. (2005); Estrada-Cuzcano et al. } \\
\text { (2011) }\end{array}$ \\
\hline & CEP290 & NPHP6 & DDR signaling & $\begin{array}{l}\text { Infantile, } \\
\text { juvenile, adult }\end{array}$ & $\begin{array}{l}\text { Retinitis pigmentosa, leber congenital amaurosis, } \\
\text { neurologic symptoms, liver disease }\end{array}$ & $\begin{array}{l}\text { Sayer et al. (2006); Valente et al. (2006); } \\
\text { Helou et al. (2007); Tory et al. (2007) }\end{array}$ \\
\hline & RPGRIP1L & NPHP8 & - & $\begin{array}{l}\text { Infantile, } \\
\text { juvenile, adult }\end{array}$ & $\begin{array}{l}\text { Retinitis pigmentosa, neurologic symptoms, liver } \\
\text { disease, polydactyly }\end{array}$ & $\begin{array}{l}\text { Arts et al. (2007); Delous et al. (2007); Wolf } \\
\text { et al. (2007) }\end{array}$ \\
\hline & TMEM67 & NPHP11 & - & $\begin{array}{l}\text { Infantile, } \\
\text { juvenile, adult }\end{array}$ & $\begin{array}{l}\text { Retinitis pigmentosa, neurologic symptoms, liver } \\
\text { disease, polydactyly }\end{array}$ & Smith U. M. et al. (2006) \\
\hline \multirow[t]{2}{*}{$\begin{array}{l}\text { Intraflagellar transport } \\
\text { complex IFT-B }\end{array}$} & IFT172 & NPHP17 & - & $\begin{array}{l}\text { Infantile, } \\
\text { juvenile, adult }\end{array}$ & $\begin{array}{l}\text { Retinitis pigmentosa, neurologic symptoms, liver } \\
\text { disease, skeletal anomalies, polydactyly }\end{array}$ & Halbritter et al. (2013a) \\
\hline & TRAF3IP1/IFT54 & SLNS9 & Microtubule dynamics & $\begin{array}{l}\text { Infantile, } \\
\text { Juvenile }\end{array}$ & $\begin{array}{l}\text { Retinitis pigmentosa, hepatic fibrosis, skeletal } \\
\text { anomalies, obesity }\end{array}$ & Bizet et al. (2015) \\
\hline \multirow[t]{2}{*}{$\begin{array}{l}\text { Intraflagellar transport } \\
\text { complex IFT-A }\end{array}$} & TTC21B/IFT139 & NPHP12 & Microtubule dynamics & $\begin{array}{l}\text { Infantile, } \\
\text { juvenile }\end{array}$ & $\begin{array}{l}\text { Neurologic symptoms, situs inversus, liver disease, } \\
\text { skeletal anomalies }\end{array}$ & Davis et al. (2011); Cong et al. (2014) \\
\hline & WDR19/IFT144 & NPHP13 & - & $\begin{array}{l}\text { Infantile, } \\
\text { juvenile }\end{array}$ & $\begin{array}{l}\text { Retinitis pigmentosa, liver disease (especially Caroli } \\
\text { disease), pancreas anomalies, skeletal anomalies }\end{array}$ & Bredrup et al. (2011) \\
\hline \multirow[t]{4}{*}{ Inversin compartment } & INVS & NPHP2 & $\begin{array}{l}\text { Cell junctions; mitotic } \\
\text { spindle poles, midbody }\end{array}$ & $\begin{array}{l}\text { Infantile, } \\
\text { juvenile }\end{array}$ & $\begin{array}{l}\text { Retinitis pigmentosa, neurologic symptoms, situs } \\
\text { inversus, congenital heart disease, liver disease }\end{array}$ & $\begin{array}{l}\text { Otto et al. (2003); O'Toole et al. (2006); Tory } \\
\text { et al. (2009) }\end{array}$ \\
\hline & NPHP3 & NPHP3 & - & $\begin{array}{l}\text { Infantile, } \\
\text { juvenile, adult }\end{array}$ & $\begin{array}{l}\text { Retinitis pigmentosa, neurologic symptoms, situs } \\
\text { inversus, congenital heart defect, liver disease }\end{array}$ & $\begin{array}{l}\text { Omran et al. (2000); Olbrich et al. (2003); } \\
\text { Bergmann et al. (2008); Tory et al. (2009) }\end{array}$ \\
\hline & NEK8 & NPHP9 & DDR and Hippo signaling & $\begin{array}{l}\text { Infantile, } \\
\text { juvenile }\end{array}$ & $\begin{array}{l}\text { Situs inversus, congenital heart disease, liver } \\
\text { disease, pancreas anomalies }\end{array}$ & Otto et al. (2008); Grampa et al. (2016) \\
\hline & ANKS6 & NPHP16 & - & $\begin{array}{l}\text { Infantile, } \\
\text { juvenile, adult }\end{array}$ & $\begin{array}{l}\text { Neurologic symptoms, situs inversus, congenital } \\
\text { heart disease, liver disease }\end{array}$ & Hoff et al. (2013); Taskiran et al. (2014) \\
\hline \multirow[t]{3}{*}{ Basal body } & SDCCAG8 & NPHP10 & DDR signaling & $\begin{array}{l}\text { Infantile, } \\
\text { juvenile, adult }\end{array}$ & $\begin{array}{l}\text { Retinitis pigmentosa, neurologic symptoms, } \\
\text { obesity, hypogenitalism }\end{array}$ & Otto et al. (2010); Watanabe et al. (2019) \\
\hline & CEP164 & NPHP15 & DDR signaling & Juvenile & $\begin{array}{l}\text { Retinitis pigmentosa, Leber congenital amaurosis, } \\
\text { neurologic symptoms, liver disease, polydactyly }\end{array}$ & Chaki et al. (2012) \\
\hline & CEP83 & NPHP18 & - & $\begin{array}{l}\text { Infantile, } \\
\text { juvenile }\end{array}$ & $\begin{array}{l}\text { Retinitis pigmentosa, neurologic symptoms, liver } \\
\text { disease }\end{array}$ & Failler et al. (2014) \\
\hline Near basal body & ADAMTS9 & NPHP21 & $\begin{array}{l}\text { Metalloproteinase function, } \\
\text { protein trafficking }\end{array}$ & $\begin{array}{l}\text { Infantile, } \\
\text { juvenile }\end{array}$ & Deafness, short stature, developmental delay & Choi et al. (2019); Nandadasa et al. (2019) \\
\hline
\end{tabular}


other IFT-A encoding genes (Perrault et al., 2012; Schmidts et al., 2013). This functional classification of NPH genes also corresponds to specific extrarenal manifestations. For example, genes encoding transition zone proteins are associated with retinal dystrophy, genes encoding InvsC proteins are associated with situs inversus and most genes encoding IFT subunits give rise to skeletal dysplasia.

Interestingly, several NPHPs have not been linked directly to ciliary functions including GLIS2 (NPHP7, see below), ZNF423 (NPHP14) and the recently discovered gene MAPKBP1 (NPHP20). However, ZNF423 has been shown to interact with CEP290 and its murine ortholog Zfp423 was shown to be involved in the transcription of ciliary genes (Chaki et al., 2012; Hong and Hamilton, 2016). Mutations in MAPKBP1 were identified in several individuals presenting with late onset juvenile or adult NPH (Macia et al., 2017; Schonauer et al., 2020; Shamseldin et al., 2020). MAPKBP1 encodes a non-ciliary scaffold protein involved in JNK signaling that localizes to mitotic spindle poles (Macia et al., 2017) as well as to the centrosome (Schonauer et al., 2020). Loss of MAPKBP1 causes increased DNA-damage response signaling as observed in other NPH subtypes [see section "DNA-Damage Response (DDR)"] but no ciliogenesis defects (Macia et al., 2017), indicating that this may represent a new cilia-independent subtype of isolated $\mathrm{NPH}$.

Of note, NPH can be a feature of syndromic ciliopathies caused by mutations in genes currently not classified as NPHP genes ( $B B S$ genes for example), resulting in more than 90 genes associated with an 'NPH-related ciliopathy' (Braun and Hildebrandt, 2017). In addition, biallelic mutations in XPNPEP3 and SLC41A1 can cause an NPH-like phenotype (O'Toole et al., 2010; Hurd et al., 2013). These genes and their functions fall outside the scope of this review.

\section{Pathophysiology Cilia-Associated Signaling Pathways}

The combination of the transition zone at the base of the cilium which limits passive diffusion of molecules and the active transport of ciliary proteins mediated by the IFT/BBSome complexes generates a closed compartment that allows a concentration of signaling molecules and rapid signal transduction independent from rest of the cell body (Hansen et al., 2020). The primary cilium therefore acts as a cellular signaling hub for chemosensory (for example GPCR) and mechanosensory (for example polycystin1/polycystin-2 receptor-ion channel complex) signaling (Spasic and Jacobs, 2017). PC have also been proposed as a source of a specific subtype of extracellular vesicles or 'ectosomes' which are likely acting as signaling messengers (Wood et al., 2013; Wang and Barr, 2016; Nager et al., 2017; Phua et al., 2017). Ciliary defects can disrupt these pathways, which in turn can impair kidney development and, more importantly in NPH, maintenance of kidney architecture and function. Cilia-dependent signal transduction pathways have been extensively reviewed elsewhere (Wheway et al., 2018; Anvarian et al., 2019). Specific cilia-associated signaling pathways are discussed in more detail in Section 
"Targeting Ciliary Signaling Pathways," which summarizes pathways that have been targeted pharmacologically in the context of NPH and/or ADPKD including mTOR, Hedgehog (Hh) and Hippo pathways. Of note, there is mounting evidence for crosstalk between ciliary signaling pathways and DNA-damage response (DDR) signaling discussed below (Wang et al., 2020).

\section{DNA-Damage Response (DDR)}

Studies over the past 10 years pointed to an important functional relationship between several NPHP gene products and DDR signaling (Slaats and Giles, 2015; Zhang J. Q. J. et al., 2019). DDR signaling is involved in the detection of DNA damage and activation of appropriate cellular responses including cell cycle arrest and activation of DNA repair mechanisms. Chaki et al. (2012) proposed that disruption of DDR signaling and consequent impairment of cell-cycle checkpoint control early in development leads to depletion of progenitor cells and dysplastic phenotypes, while milder disruptions of DDR signaling associated with hypomorphic mutations lead to a slow accumulation of DNA damage over time resulting in degenerative phenotypes such as NPH. Since the discovery of the role of ZNF423/NPHP14 and CEP164/NPHP15 in DDR signaling, this functional link has been confirmed in NPH genes CEP290/NPHP6, NEK8/NPHP9, SDCCAG8/NPHP10 and MAPKBP1/NPHP20 (Otto et al., 2008, 2010; Chaki et al., 2012; Choi et al., 2013; Airik et al., 2014; Slaats et al., 2014, 2015; Slaats and Giles, 2015; Macia et al., 2017). Interestingly, analysis of primary kidney cells from Cep290-deficient mice showed increased double-strand breaks, replication fork asymmetry and reduced fork velocity suggestive of increased replication stress, and increased levels of cyclindependent kinases (CDKs). These effects could be rescued in vitro using CDK inhibitors (Slaats et al., 2015). Cep164 knockdown in ciliated cells induced epithelial-to-mesenchymal transition (EMT) and upregulation of fibrosis-associated genes, further linking aberrant DDR signaling and S-phase arrest to tubulointerstitial fibrosis in NPH (Slaats et al., 2014). Correspondingly, MAPKBP1-deficient cells showed increased DDR, and kidney sections from MAPKBP1-related NPH patients revealed tubular atrophy and massive tubulointerstitial fibrosis (Macia et al., 2017).

\section{Polarity}

Next to nuclear functions, specific NPH-associated proteins have additional roles outside the cilium. For example, NPHP1, INVS and NPHP4 localize to and regulate cell-cell junctions (Donaldson et al., 2002; Nurnberger et al., 2002; Delous et al., 2009). NPHP1 and NPHP4 are required for timely tight junction formation and interact with the proteins PALS1 and PAR6 involved in establishment of epithelial cell polarity. Knockdown of NPHP1 and NPHP4 in MDCK cells resulted in disorganized, multi-lumen structures, and loss of cell polarity (Delous et al., 2009). Similar findings were obtained for other NPHPs in murine IMCD3 kidney cells suggesting a shared function in polarity and epithelialization (Sang et al., 2011; Ghosh et al., 2012). Interestingly, in the case of TRAF3IP1/IFT54, the observed polarity defect could be linked to an increased stability of cytoplasmic microtubules (see below) (Bizet et al., 2015). Finally, the loss of Glis2/Nphp7 causes NPH in mice through EMT driven by the expression of EMT specific genes such as SNAIL (Attanasio et al., 2007; Kim et al., 2008; Li et al., 2011). Altogether, these data suggest that the crucial roles of NPHP proteins in polarity/epithelialization likely contribute to the tubular atrophy and loss of nephrons observed in juvenile NPH.

\section{Inflammation and Senescence}

Most recently, activation of inflammation was proposed to be involved in tubulointerstitial fibrosis in NPH. NPHP1 is part of a protein complex with the kinase LKB1 that regulates cilia-controlled secretion of the chemokine CCL2, and Nphp1 silencing induced increased $\mathrm{Ccl} 2$ expression in kidney cells (Viau et al., 2018). Ccl2 has been associated with macrophage activation and disease progression in CKD (Cao et al., 2015). In addition, mononuclear infiltrates and inflammation have been described in animal models of NPH including $p c y / N p h p 3$ and Glis2/Nphp7 mice (Omran et al., 2001; Kim et al., 2008). Interestingly, Glis2/Nphp7 knockout mice were shown to have increased tubular cell senescence (Jin et al., 2020). Replicative senescence leads to secretion of pro-inflammatory cytokines and activation of the NF-kB pathway, eliciting an inflammatory response, increased senescence in neighboring cells and extensive kidney damage as demonstrated in a Glis2 knockout mouse and in other mouse models of CKD (Acosta et al., 2013; Docherty et al., 2019; Jin et al., 2020). In addition, senescent tubular epithelial cells were demonstrated to be a source of pro-fibrotic Hh ligands (Jin et al., 2019). Similar processes could contribute to tubulointerstitial damage in other subtypes of juvenile NPH with known defects in DNA-damage response signaling and cell cycle progression, although it is unknown whether tubular cell senescence is a general feature of NPH.

\section{Additional Mechanisms}

Additional cilia-independent mechanisms that have been pharmacologically targeted in NPH, including glycosphingolipids and cholesterol, and microtubule dynamics, are discussed in Section "Pharmacotherapy." Other factors were not directly targeted but have been proposed to play a role in the pathophysiology of NPH. For example, oxidative stress, the endoplasmic reticulum (ER) stress response and mitochondrial dynamics were shown to be dysregulated in the jck mouse model of NEK8/NPHP9 (Bracken et al., 2016). In addition, the $\mathrm{NPH}$-associated metalloproteinase ADAMTS9 was involved in regulation of ER-Golgi vesicular transport (Yoshina et al., 2012). However, ADAMTS9 was also shown to localize in vesicular structures near the base of the cilium and to be required for ciliogenesis, a function which appears to be distinct from its role in ER-Golgi transport (Choi et al., 2019; Nandadasa et al., 2019). The relationship between the ubiquitin-proteasome system, autophagy and primary cilia has been recently reviewed (Boukhalfa et al., 2019; Wiegering et al., 2019). Finally, increased formation of ciliary extracellular vesicle has been observed in BBS mutant cells suggesting that dysregulation of this process might contribute to ciliopathies (Nager et al., 2017; Akella et al., 2020). 
However, for most proposed mechanisms it remains unclear how individual factors contribute to specific NPH phenotypes.

\section{AVAILABLE MODEL SYSTEMS}

\section{Animal Models}

Although studies in animal models of NPH have provided abovementioned insights into pathophysiology of NPH, most models do not recapitulate the juvenile NPH phenotypes.

Zebrafish larva (24-72 h post fertilization) is a widely used organism to study renal ciliopathies. This model is low cost, fast and efficient, allowing in vivo imaging, functional studies and large-scale screening of small molecules for therapeutic approaches. Most models have been based on morpholinobased knockdown of the respective nphp genes, resulting in loss of function like conditions, typically resulting in severe developmental defects including cysts in the proximal pronephros and/or defects in the distal part of the pronephros associated with ciliary defects (Slanchev et al., 2011; Song et al., 2016; Elmonem et al., 2018). It is unlikely that fibrosis could be monitored/observed in early developmental stages in the simple pronephros which is present in the embryo; this would likely require the development of adult fish models. Generation of mutant zebrafish lines to study more slowly progressive juvenile $\mathrm{NPH}$ is limited because most described mutants show either too severe phenotypes with early death at larval stages, or no phenotype in the adults (Song et al., 2016; Macia et al., 2017), likely due to compensation mechanisms (El-Brolosy and Stainier, 2017). Interestingly, a recent study described a hypomorphic mutation in tmem67 (NPHP11), leading to ciliary defects and tubular cysts in adult zebrafish kidneys (Zhu et al., 2021), indicating that such models can be generated. Unfortunately, fibrosis was not monitored in this study.

Mouse models more accurately represent human kidney physiology and have been extensively used to characterize mechanisms underlying ciliopathies as well as CKD and associated fibrosis (Norris and Grimes, 2012; Ko and Park, 2013; Nogueira et al., 2017). However, while kidney fibrosis has been observed in mouse models of PKD and after toxic challenges, mice appear to be relatively resistant to NPH-related fibrosis. Indeed, published knockout mouse models corresponding to juvenile NPH including Nphp1, Nphp4, Nphp5 and Mapkbp1 do not show an obvious spontaneous renal phenotype (Jiang et al., 2008; Won et al., 2011; Ronquillo et al., 2016; Macia et al., 2017). Interestingly, only Glis2/Nphp7-knockout mice show a phenotype that fully represents juvenile NPH, comprising smaller kidneys with increased apoptosis, immune infiltration, fibrosis and tubular dilations on kidney sections, increased serum creatinine and polyuria (Kim et al., 2008).

Mouse models of InvsC defects (Invs, $p c y / N p h p 3$, jck/Nek8/Nphp9 and Anks6/Nphp16-mutated mice) show enlarged cystic kidneys characteristic of infantile NPH, although $p c y / N p h p 3$ and Anks6/Nphp16 mice have slow disease progression and (late stage) tubulointerstitial fibrosis (Omran et al., 2001; Bakey et al., 2015). The $p c y / N p h p 3$ mouse model, which harbors a homozygous missense mutation in
$N p h p 3$, is characterized by cysts at the corticomedullary junction (later throughout the kidney), kidney enlargement and late-stage fibrosis (Takahashi et al., 1991; Omran et al., 2001; Olbrich et al., 2003). Consequently, $p c y / N p h p 3$ mice show more resemblance to ADPKD than to juvenile NPH. This is relevant because contrary to ADPKD, cyst formation in juvenile NPH likely occurs secondary to tissue degeneration and atrophy instead of being a driving mechanism, so targetable mechanisms in both conditions could diverge (Braun and Hildebrandt, 2017; Srivastava et al., 2018). Similarly, jck/Nek8/Nphp9 mice, which show enlarged cystic kidneys and rapid disease progression, have been used as a model for (AR)PKD (Bukanov et al., 2006). These differences between human and animal phenotypes should be taken into account in pharmacological studies in NPH.

With the exception of juvenile NPHP genes mentioned above, genetic invalidation of most NPHP genes causes cystic kidney enlargement in mice (Table 2). These severe phenotypes could partly be explained by predominantly complete loss of function mutations in the mice models, which do not correspond to the (often hypomorphic) mutations in (at least) part of patients with juvenile NPH. The same limitations are found in the Lewis polycystic kidney (LPK), Wistar polycystic kidney (Wpk) and $C y$ rat models, with mutations in the orthologs of human NEK8/NPHP9, TMEM67/NPHP11 and ANKS6/NPHP16 genes, respectively. These all show rapidly progressive cystic kidney enlargement reminiscent of infantile NPH or ARPKD phenotypes (Cowley et al., 1993; Gattone et al., 2004; Brown et al., 2005; Smith U. M. et al., 2006; Phillips et al., 2007; McCooke et al., 2012; Taskiran et al., 2014).

It is important to note that the type of genetic defect in animal models (knockout/loss of function or missense mutations) and the genetic background strain with associated genetic modifiers impact the severity of the phenotype and the utility of the model for human disease, as was demonstrated in mouse models of Cep290-associated ciliopathies (Ramsbottom et al., 2015, 2020). Patient-derived in vitro systems, for example urinary renal epithelial cells (URECs), kidney tubuloids and organoids, could partly solve these issues (Schutgens et al., 2019; Steichen et al., 2020).

\section{Kidney Organoids and Tubuloids}

Primary kidney tubular epithelial organoids, or 'tubuloids', can be efficiently established from adult stem cells derived from kidney biopsy material and from URECs. Tubuloids were employed to study infectious kidney disease in a personalized fashion and test the efficacy of cystic fibrosis treatment using a forskolin (cAMP) swelling assay, illustrating its utility in (personalized) drug screening (Schutgens et al., 2019). In contrast to tubuloids, which are restricted to epithelial tubular cells, kidney organoids were generated from human induced pluripotent stem cells (iPSC) that contained different parts of the nephron (glomerulus, proximal and distal tubule) and interstitial cells (Yousef Yengej et al., 2020). While these organoids lack collecting ducts, other protocols were recently setup to generate ureteric bud-derived collecting ducts enriched organoids (Kuraoka et al., 2020; Uchimura et al., 2020). Kidney organoids were also generated from adult differentiated kidney cells which incorporated extracellular matrix (ECM) cells, 
TABLE 2 | Rodent models of NPH discussed in this review.

\begin{tabular}{|c|c|c|c|c|c|c|c|c|c|c|c|}
\hline Species & Name & $\begin{array}{l}\text { Human } \\
\text { ortholog }\end{array}$ & $\begin{array}{l}\text { Type of } \\
\text { mutation }\end{array}$ & $\begin{array}{l}\text { Renal disease } \\
\text { progression }\end{array}$ & Survival & $\begin{array}{l}\text { Tubular } \\
\text { phenotype }\end{array}$ & $\begin{array}{l}\text { Interstitial } \\
\text { phenotype }\end{array}$ & $\begin{array}{l}\text { Urine } \\
\text { concentration } \\
\text { defect }\end{array}$ & Kidney size & $\begin{array}{l}\text { Extrarenal } \\
\text { phenotypes }\end{array}$ & References \\
\hline \multirow[t]{7}{*}{ Mouse } & Nphp1 & NPHP1 & $\begin{array}{l}\text { Out-of-frame } \\
\text { deletion exon } \\
20\end{array}$ & NA & Normal & - & - & NA & Normal & Male infertility & $\begin{array}{l}\text { Jiang et al. } \\
\text { (2008) }\end{array}$ \\
\hline & Invs & INVS/NPHP2 & $\begin{array}{l}\text { Out-of-frame } \\
\text { deletion exon } \\
\text { 3-11 }\end{array}$ & $\begin{array}{l}\text { Rapid (kidney } \\
\text { failure around } \\
\text { age } 1 \text { week) }\end{array}$ & Around 1 week & $\begin{array}{l}\text { Absence of } \\
\text { tubular atrophy } \\
\text { and tubular } \\
\text { basement } \\
\text { membrane } \\
\text { irregularities; } \\
\text { corticomedullary } \\
\text { cysts arising } \\
\text { from proximal } \\
\text { tubule and } \\
\text { collecting duct; }\end{array}$ & - & NA & Enlarged & $\begin{array}{l}\text { Situs inversus, } \\
\text { biliary } \\
\text { obstruction/ } \\
\text { atresia }\end{array}$ & $\begin{array}{l}\text { Yokoyama et al. } \\
\text { (1993); Lowe } \\
\text { et al. (1996); } \\
\text { Mochizuki et al. } \\
\text { (1998); Morgan } \\
\text { et al. (1998); } \\
\text { Phillips et al. } \\
\text { (2004) }\end{array}$ \\
\hline & $\begin{array}{l}\text { Polycystic } \\
\text { kidney disease } \\
\text { (Pcy) }\end{array}$ & NPHP3 & Missense & $\begin{array}{l}\text { Slow (kidney } \\
\text { failure in adult } \\
\text { mice) }\end{array}$ & NA & $\begin{array}{l}\text { Tubular atrophy } \\
\text { and tubular } \\
\text { basement } \\
\text { membrane } \\
\text { thickening; } \\
\text { corticomedullary } \\
\text { cysts followed } \\
\text { by entire } \\
\text { kidney, tubular } \\
\text { dilations arising } \\
\text { predominantly } \\
\text { from distal } \\
\text { tubules and } \\
\text { collecting duct }\end{array}$ & $\begin{array}{l}\text { Tubulointerstitial } \\
\text { fibrosis and } \\
\text { inflammation } \\
\text { (late stage) }\end{array}$ & NA & Enlarged & $\begin{array}{l}\text { Cerebral } \\
\text { vascular } \\
\text { aneurysms }\end{array}$ & $\begin{array}{l}\text { Takahashi et al. } \\
\text { (1991); Omran } \\
\text { et al. (2001); } \\
\text { Olbrich et al. } \\
\text { (2003) }\end{array}$ \\
\hline & Nphp4 & NPHP4 & Missense & NA & Normal & - & - & NA & Normal & $\begin{array}{l}\text { Retinal disease, } \\
\text { male infertility }\end{array}$ & $\begin{array}{l}\text { Won et al. } \\
\text { (2011) }\end{array}$ \\
\hline & lacb1 & $\begin{array}{l}\text { IQCB1/ } \\
\text { NPHP5 }\end{array}$ & $\begin{array}{l}\text { Gene trap } \\
\text { leading to loss } \\
\text { of function }\end{array}$ & NA & NA & - & - & NA & Normal & Retinal disease & $\begin{array}{l}\text { Ronquillo et al. } \\
\text { (2016) }\end{array}$ \\
\hline & Сep290 & $\begin{array}{l}\text { CEP290/ } \\
\text { NPHP6 }\end{array}$ & $\begin{array}{l}\text { Gene trap } \\
\text { inserted in } \\
\text { intron } 25 \\
\text { leading to } \\
\text { premature stop } \\
\text { codon }\end{array}$ & NA & $\begin{array}{l}\text { 129/Ola } \\
\text { background: } \\
\text { survival past } \\
\text { age } 1 \text { year }\end{array}$ & $\begin{array}{l}\text { Small cysts in } \\
\text { cortex arising } \\
\text { from collecting } \\
\text { duct }\end{array}$ & - & $\begin{array}{l}\text { Evidence of } \\
\text { polyuria and } \\
\text { polydipsia }\end{array}$ & NA & $\begin{array}{l}\text { Retinal disease, } \\
\text { cerebral } \\
\text { abnormalities }\end{array}$ & $\begin{array}{l}\text { Hynes et al. } \\
\text { (2014) }\end{array}$ \\
\hline & & & $\begin{array}{l}\text { Rd16: deletion } \\
\text { of exons } 35 \text { to } \\
39\end{array}$ & NA & NA & - & - & NA & Normal & Retinal disease & $\begin{array}{l}\text { Chang et al. } \\
\text { (2006) }\end{array}$ \\
\hline
\end{tabular}


TABLE 2 | Continued

\begin{tabular}{|c|c|c|c|c|c|c|c|c|c|c|c|}
\hline Species & Name & $\begin{array}{l}\text { Human } \\
\text { ortholog }\end{array}$ & $\begin{array}{l}\text { Type of } \\
\text { mutation }\end{array}$ & $\begin{array}{l}\text { Renal disease } \\
\text { progression }\end{array}$ & Survival & $\begin{array}{l}\text { Tubular } \\
\text { phenotype }\end{array}$ & $\begin{array}{l}\text { Interstitial } \\
\text { phenotype }\end{array}$ & $\begin{array}{l}\text { Urine } \\
\text { concentration } \\
\text { defect }\end{array}$ & Kidney size & $\begin{array}{l}\text { Extrarenal } \\
\text { phenotypes }\end{array}$ & References \\
\hline & & & Knockout & Slow & $\begin{array}{l}\text { Mixed C57BL/6 } \\
\text { and 129/SvJ } \\
\text { background: } \\
80 \% \text { lethality } \\
\text { within first } \\
\text { weeks due to } \\
\text { hydrocephalus; } \\
\text { surviving mice } \\
\text { lived until age } \\
2 \text { years }\end{array}$ & $\begin{array}{l}\text { Corticomedullary } \\
\text { cysts develop } \\
\text { after } 12 \text { months }\end{array}$ & - & NA & Enlarged & $\begin{array}{l}\text { Retinal disease, } \\
\text { cerebral and } \\
\text { cerebellar } \\
\text { abnormalities }\end{array}$ & $\begin{array}{l}\text { Rachel et al. } \\
\text { (2015) }\end{array}$ \\
\hline & & & $\begin{array}{l}\text { Gene trap } \\
\text { inserted in } \\
\text { intron } 25 \\
\text { leading to } \\
\text { premature stop } \\
\text { codon }\end{array}$ & Rapid & $\begin{array}{l}\text { C57BL/6 and } \\
\text { 129/SvJ } \\
\text { backgrounds: } \\
\text { majority dies } \\
\text { prenatally; mice } \\
\text { that survive to } \\
\text { age 2-3 weeks } \\
\text { show severe } \\
\text { cystic kidney } \\
\text { disease }\end{array}$ & $\begin{array}{l}\text { Loss of tubules } \\
\text { (not further } \\
\text { specified); } \\
\text { corticomedullary } \\
\text { cysts }\end{array}$ & Cellular infiltrate & NA & Enlarged & $\begin{array}{l}\text { Retinal disease, } \\
\text { hepatic pallor }\end{array}$ & \\
\hline & Glis2 & GLIS2/NPHP7 & Knockout & NA & $\begin{array}{l}>40 \% \text { lethality } \\
\text { by age } \\
10 \text { months }\end{array}$ & $\begin{array}{l}\text { Atrophy of } \\
\text { proximal } \\
\text { tubules, } \\
\text { minimal tubular } \\
\text { basement } \\
\text { membrane } \\
\text { thickening; } \\
\text { corticomedullary } \\
\text { and glomerular } \\
\text { cysts }\end{array}$ & $\begin{array}{l}\text { Tubulointerstitial } \\
\text { inflammation } \\
\text { and fibrosis } \\
\text { starting at age } \\
8 \text { weeks }\end{array}$ & NA & Reduced & - & $\begin{array}{l}\text { Attanasio et al. } \\
\text { (2007); Kim } \\
\text { et al. (2008) }\end{array}$ \\
\hline & Rpgrip1/ (Ftm) & $\begin{array}{l}\text { RPGRIP1L } \\
\text { NPHP8 }\end{array}$ & Knockout & NA & $\begin{array}{l}\text { Embryonically } \\
\text { lethal }\end{array}$ & $\begin{array}{l}\text { Cortical } \\
\text { microcysts } \\
\text { arising from } \\
\text { proximal tubule } \\
\text { at } 18.5 \text { days } \\
\text { post } \\
\text { conception }\end{array}$ & - & NA & NA & $\begin{array}{l}\text { Exencephaly, } \\
\text { microphthalmia, } \\
\text { situs inversus, } \\
\text { liver } \\
\text { abnormalities, } \\
\text { polydactyly }\end{array}$ & $\begin{array}{l}\text { Delous et al. } \\
(2007)\end{array}$ \\
\hline
\end{tabular}


TABLE 2 | Continued

\begin{tabular}{|c|c|c|c|c|c|c|c|c|c|c|c|}
\hline Species & Name & $\begin{array}{l}\text { Human } \\
\text { ortholog }\end{array}$ & $\begin{array}{l}\text { Type of } \\
\text { mutation }\end{array}$ & $\begin{array}{l}\text { Renal disease } \\
\text { progression }\end{array}$ & Survival & $\begin{array}{l}\text { Tubular } \\
\text { phenotype }\end{array}$ & $\begin{array}{l}\text { Interstitial } \\
\text { phenotype }\end{array}$ & $\begin{array}{l}\text { Urine } \\
\text { concentration } \\
\text { defect }\end{array}$ & Kidney size & $\begin{array}{l}\text { Extrarenal } \\
\text { phenotypes }\end{array}$ & References \\
\hline & $\begin{array}{l}\text { Juvenile cystic } \\
\text { kidneys (Jck) }\end{array}$ & NEK8/NPHP9 & Missense & NA & $\begin{array}{l}100 \% \text { lethality } \\
\text { by age } \\
25 \text { weeks in } \\
\text { males and age } \\
80 \text { weeks in } \\
\text { females }\end{array}$ & $\begin{array}{l}\text { Basement } \\
\text { membrane } \\
\text { disruptions; } \\
\text { corticomedullary } \\
\text { cysts followed } \\
\text { by entire } \\
\text { kidney, arising } \\
\text { from collecting } \\
\text { duct, in later } \\
\text { stage also from } \\
\text { distal tubule } \\
\text { and loop of } \\
\text { Henle }\end{array}$ & - & NA & Enlarged & - & $\begin{array}{l}\text { Atala et al. } \\
\text { (1993); Liu et al. } \\
\text { (2002); Smith } \\
\text { L. A. et al. } \\
\text { (2006); Otto } \\
\text { et al. (2008) }\end{array}$ \\
\hline & & & Knockout & NA & $\begin{array}{l}\text { Die within hours } \\
\text { after birth due } \\
\text { to congenital } \\
\text { heart defect }\end{array}$ & $\begin{array}{l}\text { Proximal tubule } \\
\text { dilation; } \\
\text { glomerular } \\
\text { cysts and (few } \\
\text { cysts develop } \\
\text { in kidney } \\
\text { explant culture) }\end{array}$ & - & NA & Normal & $\begin{array}{l}\text { Situs inversus, } \\
\text { cardiac } \\
\text { anomalies }\end{array}$ & $\begin{array}{l}\text { Manning et al. } \\
\text { (2013) }\end{array}$ \\
\hline & Sdccag8 & $\begin{array}{l}\text { SDCCAG8/ } \\
\text { NPHP10 }\end{array}$ & $\begin{array}{l}\text { Gene trap } \\
\text { leading to loss } \\
\text { of function }\end{array}$ & Slow & $\begin{array}{l}\text { Survival } \\
>250 \text { days }\end{array}$ & $\begin{array}{l}\text { Initial cortical } \\
\text { cysts followed } \\
\text { by } \\
\text { corticomedullary } \\
\text { cysts, arising } \\
\text { from distal } \\
\text { convoluted } \\
\text { tubule and } \\
\text { collecting duct; } \\
\text { glomerular } \\
\text { cysts }\end{array}$ & $\begin{array}{l}\text { Tubulointerstitial } \\
\text { fibrosis (late } \\
\text { stage) }\end{array}$ & NA & $\begin{array}{l}\text { Enlarged (late } \\
\text { stage) }\end{array}$ & Retinal disease & $\begin{array}{l}\text { Airik et al. } \\
\text { (2014) }\end{array}$ \\
\hline & $\begin{array}{l}\text { Bilateral } \\
\text { polycystic } \\
\text { kidneys (Bpck) }\end{array}$ & $\begin{array}{l}\text { TMEM67/ } \\
\text { NPHP11 }\end{array}$ & Deletion & $\begin{array}{l}\text { Rapid (kidney } \\
\text { failure around } \\
\text { age } 3 \text { weeks) }\end{array}$ & $\begin{array}{l}\text { Survive to age } \\
3 \text { weeks }\end{array}$ & $\begin{array}{l}\text { Corticomedullary } \\
\text { cysts arising } \\
\text { from distal } \\
\text { tubule and } \\
\text { collecting duct, } \\
\text { later mild } \\
\text { dilation of some } \\
\text { proximal } \\
\text { tubules }\end{array}$ & - & NA & Enlarged & $\begin{array}{l}\text { Hydrocephalus, } \\
\text { spermatogenesis } \\
\text { defects }\end{array}$ & $\begin{array}{l}\text { Cook et al. } \\
\text { (2009) }\end{array}$ \\
\hline
\end{tabular}


TABLE 2 | Continued

\begin{tabular}{|c|c|c|c|c|c|c|c|c|c|c|c|}
\hline Species & Name & $\begin{array}{l}\text { Human } \\
\text { ortholog }\end{array}$ & $\begin{array}{l}\text { Type of } \\
\text { mutation }\end{array}$ & $\begin{array}{l}\text { Renal disease } \\
\text { progression }\end{array}$ & Survival & $\begin{array}{l}\text { Tubular } \\
\text { phenotype }\end{array}$ & $\begin{array}{l}\text { Interstitial } \\
\text { phenotype }\end{array}$ & $\begin{array}{l}\text { Urine } \\
\text { concentration } \\
\text { defect }\end{array}$ & Kidney size & $\begin{array}{l}\text { Extrarenal } \\
\text { phenotypes }\end{array}$ & References \\
\hline & \multirow[t]{2}{*}{ Ttc21b (Thm1) } & $\begin{array}{l}\text { TTC21B/ } \\
\text { NPHP12 }\end{array}$ & $\begin{array}{l}\text { Loss of function } \\
\text { mutation }\end{array}$ & NA & $\begin{array}{l}\text { Embryonically } \\
\text { lethal }\end{array}$ & $\begin{array}{l}\text { Cystic dilations } \\
\text { of glomeruli, } \\
\text { proximal } \\
\text { tubules and } \\
\text { ascending } \\
\text { loops of Henle }\end{array}$ & - & NA & NA & - & $\begin{array}{l}\text { Tran et al. } \\
(2008,2014)\end{array}$ \\
\hline & & & $\begin{array}{l}\text { Conditional } \\
\text { Ttc21b } \\
\text { knockout using } \\
\text { ROSA26Cre } \\
\text { ERT+ }\end{array}$ & $\begin{array}{l}\text { Rapid (cystic } \\
\text { kidney disease } \\
\text { and elevated } \\
\text { BUN by age } \\
6 \text { weeks when } \\
\text { Ttc21b is } \\
\text { inactivated } \\
\text { before P12-14) }\end{array}$ & NA & $\begin{array}{l}\text { Cortical cysts } \\
\text { arising from } \\
\text { proximal tubule, } \\
\text { loop of Henle } \\
\text { and collecting } \\
\text { duct }\end{array}$ & - & NA & Enlarged & - & $\begin{array}{l}\text { Tran et al. } \\
\text { (2014) }\end{array}$ \\
\hline & Cep164 & $\begin{array}{l}\text { CEP164/ } \\
\text { NPHP15 }\end{array}$ & $\begin{array}{l}\text { Kidney-specific } \\
\text { Cep164 } \\
\text { inactivation } \\
\text { using } \\
\text { Hoxb7-Cre } \\
\text { (collecting duct) }\end{array}$ & $\begin{array}{l}\text { Rapid (kidney } \\
\text { failure around } \\
3 \text { weeks) }\end{array}$ & $\begin{array}{l}\text { Median survival } \\
25 \text { days }\end{array}$ & $\begin{array}{l}\text { Cysts in } \\
\text { medulla } \\
\text { followed by } \\
\text { cortex and } \\
\text { entire kidney, } \\
\text { arising from } \\
\text { collecting duct }\end{array}$ & - & NA & Enlarged & - & $\begin{array}{l}\text { Airik et al. } \\
\text { (2019) }\end{array}$ \\
\hline & Anks6 & $\begin{array}{l}\text { ANKS6/ } \\
\text { NPHP16 }\end{array}$ & $\begin{array}{l}\text { Missense } \\
\text { mutation }\end{array}$ & Slow & $\begin{array}{l}\text { Survival to age } \\
18 \text { months }\end{array}$ & $\begin{array}{l}\text { Cysts arising } \\
\text { from collecting } \\
\text { duct, thick } \\
\text { ascending limb } \\
\text { of loop of } \\
\text { Henle, to a } \\
\text { lesser extent } \\
\text { from distal } \\
\text { tubule; } \\
\text { glomerular } \\
\text { cysts }\end{array}$ & $\begin{array}{l}\text { Interstitial } \\
\text { fibrosis }\end{array}$ & NA & Enlarged & - & $\begin{array}{l}\text { Bakey et al. } \\
\text { (2015) }\end{array}$ \\
\hline & Mapkbp1 & $\begin{array}{l}\text { MAPKBP1/ } \\
\text { NPHP2O }\end{array}$ & Knockout & NA & Normal & - & - & NA & Normal & - & $\begin{array}{l}\text { Macia et al. } \\
(2017)\end{array}$ \\
\hline & Ofd1 & OFD1 & $\begin{array}{l}\text { Kidney-specific } \\
\text { Ofd1 } \\
\text { inactivation } \\
\text { using Ksp-Cre } \\
\text { (distal tubule } \\
\text { and collecting } \\
\text { duct) }\end{array}$ & $\begin{array}{l}\text { Severely } \\
\text { impaired kidney } \\
\text { function at age } \\
1-3 \text { months }\end{array}$ & $\begin{array}{l}\text { Animals } \\
\text { sacrificed at } \\
\text { age } 3 \text { months }\end{array}$ & $\begin{array}{l}\text { Cysts in } \\
\text { medulla } \\
\text { followed by } \\
\text { cortex, arising } \\
\text { from distal } \\
\text { tubules; later } \\
\text { glomerular } \\
\text { cysts and } \\
\text { proximal } \\
\text { tubular cysts }\end{array}$ & - & NA & Enlarged & - & $\begin{array}{l}\text { Zullo et al. } \\
\text { (2010) }\end{array}$ \\
\hline
\end{tabular}


TABLE 2 | Continued

\begin{tabular}{|c|c|c|c|c|c|c|c|c|c|c|c|}
\hline Species & Name & Human ortholog & $\begin{array}{l}\text { Type of } \\
\text { mutation }\end{array}$ & $\begin{array}{l}\text { Renal disease } \\
\text { progression }\end{array}$ & Survival & $\begin{array}{l}\text { Tubular } \\
\text { phenotype }\end{array}$ & $\begin{array}{l}\text { Interstitial } \\
\text { phenotype }\end{array}$ & $\begin{array}{l}\text { Urine } \\
\text { concentration } \\
\text { defect }\end{array}$ & Kidney size & $\begin{array}{l}\text { Extrarenal } \\
\text { phenotypes }\end{array}$ & References \\
\hline & Ahi1 & $A H / 1$ & Knockout & $\begin{array}{l}\text { Slow (kidney } \\
\text { function } \\
\text { impairment at } \\
\text { age } 1 \text { year or } \\
\text { older) }\end{array}$ & $\begin{array}{l}80 \% \text { did not } \\
\text { survive into } \\
\text { adulthood }\end{array}$ & $\begin{array}{l}\text { Tubular } \\
\text { basement } \\
\text { membrane } \\
\text { disruption and } \\
\text { thickening; } \\
\text { corticomedullary } \\
\text { microcysts and } \\
\text { tubular } \\
\text { dilations, mainly } \\
\text { arising from } \\
\text { proximal tubule }\end{array}$ & $\begin{array}{l}\text { Interstitial cell } \\
\text { infiltrate and } \\
\text { fibrosis }\end{array}$ & $\begin{array}{l}\text { Urinary } \\
\text { concentration } \\
\text { defect }\end{array}$ & $\begin{array}{l}\text { Reduced by } \\
\text { age } 5 \text { months }\end{array}$ & - & $\begin{array}{l}\text { Lancaster et al. } \\
\text { (2009) }\end{array}$ \\
\hline \multirow[t]{4}{*}{$\begin{array}{l}\text { Non- } \\
\text { orthologous } \\
\text { mouse models }\end{array}$} & Fan1 & FAN1 & $\begin{array}{l}\text { Knockout mice } \\
\text { treated with } \\
2 \mathrm{mg} / \mathrm{kg} \\
\text { cisplatin }\end{array}$ & $\begin{array}{l}\text { Rapid (kidney } \\
\text { failure within } \\
5 \text { weeks after } \\
\text { start of } \\
\text { treatment) }\end{array}$ & NA & $\begin{array}{l}\text { Tubular } \\
\text { basement } \\
\text { membrane } \\
\text { thickening; } \\
\text { tubular dilation; } \\
\text { karyomegalic } \\
\text { nuclei in } \\
\text { proximal tubule }\end{array}$ & $\begin{array}{l}\text { Tubulointerstitial } \\
\text { inflammation } \\
\text { and fibrosis }\end{array}$ & NA & NA & $\begin{array}{l}\text { Bone marrow } \\
\text { failure }\end{array}$ & $\begin{array}{l}\text { Airik et al. } \\
\text { (2016) }\end{array}$ \\
\hline & Prkar1a & PRKAR1A & $\begin{array}{l}\text { Kidney-specific } \\
\text { Prka1a } \\
\text { inactivation } \\
\text { using } \\
\text { Pkhd1-Cre } \\
\text { (collecting duct) }\end{array}$ & NA & $\begin{array}{l}\text { Animals } \\
\text { sacrificed at } \\
\text { age } 3 \text { months }\end{array}$ & $\begin{array}{l}\text { Small cysts } \\
\text { primarily arising } \\
\text { from distal } \\
\text { tubule and } \\
\text { collecting duct }\end{array}$ & $\begin{array}{l}\text { Interstitial } \\
\text { fibrosis }\end{array}$ & NA & $\begin{array}{l}\text { Normal } \\
\text { (enlarged in } \\
2 / 16 \text { mice) }\end{array}$ & - & Ye et al. (2017) \\
\hline & $L k b 1$ & STK11/LKB1 & $\begin{array}{l}\text { Kidney-specific } \\
\text { Lkb1 } \\
\text { inactivation } \\
\text { using Ksp-Cre } \\
\text { (distal tubule } \\
\text { and collecting } \\
\text { duct) }\end{array}$ & $\begin{array}{l}\text { Rapid (kidney } \\
\text { failure around } \\
\text { age } 5 \text { weeks) }\end{array}$ & $\begin{array}{l}50 \% \text { survival } \\
\text { around age } \\
11 \text { months }\end{array}$ & $\begin{array}{l}\text { Tubular } \\
\text { basement } \\
\text { membrane } \\
\text { thickening, } \\
\text { tubular dilation, } \\
\text { corticomedullary } \\
\text { cysts at late } \\
\text { stage }\end{array}$ & $\begin{array}{l}\text { Tubulointerstitial } \\
\text { inflammation } \\
\text { and fibrosis }\end{array}$ & $\begin{array}{l}\text { Impaired urine } \\
\text { concentration } \\
\text { at age } 5 \text { weeks }\end{array}$ & $\begin{array}{l}\text { Reduced size } \\
\text { at age } 5 \text { weeks }\end{array}$ & - & $\begin{array}{l}\text { Viau et al. } \\
\text { (2018) }\end{array}$ \\
\hline & Aatf & AATF & $\begin{array}{l}\text { Kidney-specific } \\
\text { Aatf } \\
\text { inactivation } \\
\text { using Ksp-Cre } \\
\text { (distal tubule } \\
\text { and collecting } \\
\text { duct) }\end{array}$ & $\begin{array}{l}\text { Rapid (kidney } \\
\text { failure around } \\
\text { age } 10 \text { weeks) }\end{array}$ & $\begin{array}{l}\text { Survival } \\
10-15 \text { weeks }\end{array}$ & $\begin{array}{l}\text { Tubular } \\
\text { basement } \\
\text { membrane } \\
\text { disruption and } \\
\text { thickening; } \\
\text { corticomedullary } \\
\text { cysts arising } \\
\text { from distal } \\
\text { tubule, later } \\
\text { glomerular } \\
\text { cysts }\end{array}$ & $\begin{array}{l}\text { Interstitial } \\
\text { fibrosis }\end{array}$ & $\begin{array}{l}\text { Urinary } \\
\text { concentration } \\
\text { defect }\end{array}$ & $\begin{array}{l}\text { Reduced size } \\
\text { at age } \\
10 \text { weeks }\end{array}$ & - & $\begin{array}{l}\text { Jain et al. } \\
\text { (2019) }\end{array}$ \\
\hline
\end{tabular}


TABLE 2 | Continued

\begin{tabular}{|c|c|c|c|c|c|c|c|c|c|c|c|}
\hline Species & Name & Human ortholog & $\begin{array}{l}\text { Type of } \\
\text { mutation }\end{array}$ & $\begin{array}{l}\text { Renal disease } \\
\text { progression }\end{array}$ & Survival & $\begin{array}{l}\text { Tubular } \\
\text { phenotype }\end{array}$ & $\begin{array}{l}\text { Interstitial } \\
\text { phenotype }\end{array}$ & $\begin{array}{l}\text { Urine } \\
\text { concentration } \\
\text { defect }\end{array}$ & Kidney size & $\begin{array}{l}\text { Extrarenal } \\
\text { phenotypes }\end{array}$ & References \\
\hline \multirow[t]{4}{*}{ Rat } & $\begin{array}{l}\text { Lewis } \\
\text { polycystic } \\
\text { kidney (LPK) }\end{array}$ & NEK8/NPHP9 & Missense & $\begin{array}{l}\text { Slow (kidney } \\
\text { failure around } \\
\text { 12-24 weeks) }\end{array}$ & $\begin{array}{l}\text { No survival } \\
\text { beyond age } \\
26 \text { weeks }\end{array}$ & $\begin{array}{l}\text { Corticomedullary } \\
\text { cysts, } \\
\text { predominantly } \\
\text { arising from } \\
\text { collecting duct }\end{array}$ & $\begin{array}{l}\text { Tubulointerstitial } \\
\text { inflammation } \\
\text { and fibrosis }\end{array}$ & NA & Enlarged & - & $\begin{array}{l}\text { Phillips et al. } \\
\text { (2007); } \\
\text { McCooke et al. } \\
\text { (2012) }\end{array}$ \\
\hline & $\begin{array}{l}\text { Wistar } \\
\text { polycystic } \\
\text { kidney (Wpk) }\end{array}$ & TMEM67/NPHP11 & Missense & $\begin{array}{l}\text { Rapid (kidney } \\
\text { failure around } \\
\text { age } 3 \text { weeks) }\end{array}$ & NA & $\begin{array}{l}\text { Cysts in } \\
\text { proximal tubule } \\
\text { and collecting } \\
\text { duct }\end{array}$ & - & NA & Enlarged & $\begin{array}{l}\text { Cerebral } \\
\text { abnormalities, } \\
\text { hypoplastic } \\
\text { spleen }\end{array}$ & $\begin{array}{l}\text { Gattone et al. } \\
\text { (2004); Smith } \\
\text { U. M. et al. } \\
\text { (2006) }\end{array}$ \\
\hline & Cy & ANKS6/NPHP16 & Missense & $\begin{array}{l}\text { Homozygous: } \\
\text { rapid (kidney } \\
\text { failure around } \\
\text { age } 3 \text { weeks) }\end{array}$ & $\begin{array}{l}\text { Survive to age } \\
3 \text { weeks }\end{array}$ & $\begin{array}{l}\text { Cysts in cortex } \\
\text { and outer } \\
\text { medulla }\end{array}$ & - & NA & Enlarged & - & $\begin{array}{l}\text { Cowley et al. } \\
\text { (1993); Brown } \\
\text { et al. (2005) }\end{array}$ \\
\hline & & & Missense & $\begin{array}{l}\text { Heterozygous: } \\
\text { slow }\end{array}$ & $\begin{array}{l}\text { Males die of } \\
\text { kidney failure } \\
\text { within } 1 \text { year, } \\
\text { females survive } \\
\text { past } 1 \text { year }\end{array}$ & $\begin{array}{l}\text { Thickened } \\
\text { tubular } \\
\text { basement } \\
\text { membranes; } \\
\text { dilatations of } \\
\text { proximal and } \\
\text { distal tubule } \\
\text { and collecting } \\
\text { duct }\end{array}$ & $\begin{array}{l}\text { Tubulointerstitial } \\
\text { fibrosis and } \\
\text { inflammation }\end{array}$ & NA & $\begin{array}{l}\text { Moderately } \\
\text { enlarged }\end{array}$ & - & $\begin{array}{l}\text { Cowley et al. } \\
\text { (1993); Brown } \\
\text { et al. (2005) }\end{array}$ \\
\hline
\end{tabular}

Severity of the phenotype can depend on background strain. Presence of a renal phenotype was not reported for mouse mode/s of WDR19/NPHP13, ZNF423/NPHP14, IFT172/NPHP17, DCDC2/NPHP19 and ADAMTS9/NPHP21 (Alcaraz et al., 2006; Cheng et al., 2007; Gorivodsky et al., 2009; Kern et al., 2010; Wang et al., 2011; Ashe et al., 2012; Dubail et al., 2014; Nandadasa et al., 2015; Schueler et al., 2015; Casoni et al., 2017). For CEP83/NPHP18, a cortical radial glial progenitor cell-specific conditional knockout was created that did not permit study of renal phenotypes (Shao et al., 2020). NPH can be a feature of other syndromic ciliopathies. Corresponding mouse models were not included in this table unless they were discussed in the main text. NA, not available/not reported. 
making them suitable to study interstitial phenotypes (Takasato et al., 2015; Ding et al., 2020). Although kidney organoids have mainly been studied as models of cyst development (ADPKD and ARPKD), with forskolin treatment inducing cystogenesis (Cruz et al., 2017; Low et al., 2019; Kuraoka et al., 2020), they have also been used to monitor fibrosis and myofibroblasts expansion upon IL-1 $\beta$ treatment (Lemos et al., 2018).

Limitations are that kidney organoids do not recapitulate the overall kidney morphology and do not take into account interactions between organs including vascularization of the glomeruli (unless they are transplanted into a host organism), an essential step required for generation of intratubular flow. In addition, only one study reported the generation of iPSCsderived kidney organoid in the context of an NPH-related condition. The generated organoids were used as a source of tubular epithelial cells in which transciptomic and functional analyses confirmed major defects in polarity (Forbes et al., 2018), as previously reported for other NPHP genes in kidney epithelial cell lines (see section "Polarity"). However, these organoids did not show a cystic phenotype (Forbes et al., 2018). Further improvement of NPH patient-derived kidney organoids is required before these can be used in drug tests.

\section{PHARMACOTHERAPY}

\section{Hypothesis-Driven Therapies}

In NPH, pharmacological targeting of the defective gene product is challenging because NPH-associated mutations typically lower expression of the protein and/or result in expression of a truncated protein. This is especially the case for homozygous deletion of NPHP1, the most frequent genetic event in NPH. Possibilities for targeted therapies therefore include enhancing stability/function of remaining mutated NPHP protein and/or of the complex in which it functions, or targeting the signaling pathway in which the protein plays a role, downstream pathophysiological mechanisms or modifier gene products (Kim and Kim, 2019).

Despite efforts to uncover specific approaches, many drugs investigated for the treatment of NPH were discovered in animal models of other renal ciliopathies, mainly ADPKD. ADPKD is the most prevalent inherited kidney disease and has a partly shared pathophysiology with NPH (cysts and fibrosis). As a result, important findings in the ADPKD field may be relevant for NPH.

Of note, some therapies listed below are aimed at prevention of NPH manifestations. While this approach in animal models could provide important insights, in practice most patients are symptomatic upon diagnosis therefore precluding any preventive treatment until the identification of relevant biomarkers in asymptomatic individuals.

\section{Targeting Ciliary Signaling Pathways Targeting GPCR/cAMP signaling}

G-protein coupled receptors are a large family of signaling receptors, many of which localize to the ciliary membrane (Mykytyn and Askwith, 2017). Examples include odor receptors in olfactory neurons, rhodopsin in photoreceptors and smoothened (SMO) in various tissues. Key ciliary GPCRs are positively coupled to adenylate cyclases (AC) through $\mathrm{G} \alpha \mathrm{S}$ and their activation leads to production of cyclic AMP (cAMP), a key signaling intermediate which was shown to drive renal cyst formation in ADPKD and other renal ciliopathies (MangooKarim et al., 1989; Ye and Grantham, 1993; Ghosh et al., 2012). Indeed, polycystin-2 was shown to interact with and inhibit AC while activating phosphodiesterase activity both resulting in a decreased amount of cAMP. In addition to the loss of this negative regulation in $\mathrm{PKD}$ conditions, dysregulation of calcium signaling may also contribute to elevated levels of cAMP through overactivation of ciliary AC and cAMP production leading to increased downstream signaling and cell proliferation (Sussman et al., 2020). Interestingly, NEK8/NPHP9 was shown to positively regulate polycystin-2 expression and ciliary localization (Smith L. A. et al., 2006; Sohara et al., 2008; Manning et al., 2013), in agreement with the cystic kidneys observed in infantile NPH linked to mutations in InvsC genes.

One of the main targetable sources of cAMP is the vasopressin receptor type $2(\mathrm{~V} 2 \mathrm{R})$ encoded by $A V P R 2 . \mathrm{V} 2 \mathrm{R}$ is an important GPCR in renal physiology that localizes to the basolateral membrane and to cilia of renal tubular epithelial cells (Sherpa et al., 2019). Activation of V2R by vasopressin leads to cAMPdependent activation of PKA which in turn phosphorylates aquaporin 2 (AQP2), causing its translocation to the apical membrane where it controls water reabsorption, and upregulates AQP2 transcription (Figure 2A) (Nielsen et al., 2002). Disruption of this pathway could explain the urine concentration defect observed in NPH which partially mimics nephrogenic diabetes insipidus caused by mutations in AVPR2 (Rosenthal et al., 1992; Van den Ouweland et al., 1992). Indeed, AQP2 targeting to the apical membrane in response to vasopressin was shown to be defective in human collecting duct cells in the context of Bardet-Biedl syndrome (Marion et al., 2011). Alternatively, decreased water reabsorption could result from disruption of tubular architecture and medullary osmotic gradient through defects in cell junctions and fibrosis as in other CKD (Krishnan et al., 2008; Bockenhauer et al., 2010).

In addition to its role in water reabsorption, V2R was involved in cystic kidney diseases (PKD). Its activation was shown to stimulate cyst growth through several cAMP-mediated mechanisms including activation of PKA leading to $\mathrm{Cl}^{-}$dependent fluid secretion by cystic cells, and extracellular signalregulated protein kinase (ERK) pathway-induced epithelial cell proliferation (Yamaguchi et al., 2000; Reif et al., 2011; Sherpa et al., 2019). The use of V2R antagonists such as tolvaptan, which inhibits $\mathrm{V} 2 \mathrm{R}$ signaling reducing cAMP levels in renal collecting duct cells (Figure 2A), has proven to be a successful strategy in ADPKD. This approach was first shown to markedly reduce renal cyst formation and fibrosis and limit disease progression in a polycystic kidney rat model of ARPKD (PCK) and the $p c y / N p h p 3$ mouse model (Gattone et al., 2003). The V2R antagonist OPC31260 was subsequently shown to mitigate cystogenesis and renal function decline in a mouse model of ADPKD (Torres et al., 2004). Tolvaptan was shown to be effective in clinical trials (Torres et al., 2012, 2017, 2018) and is the first 

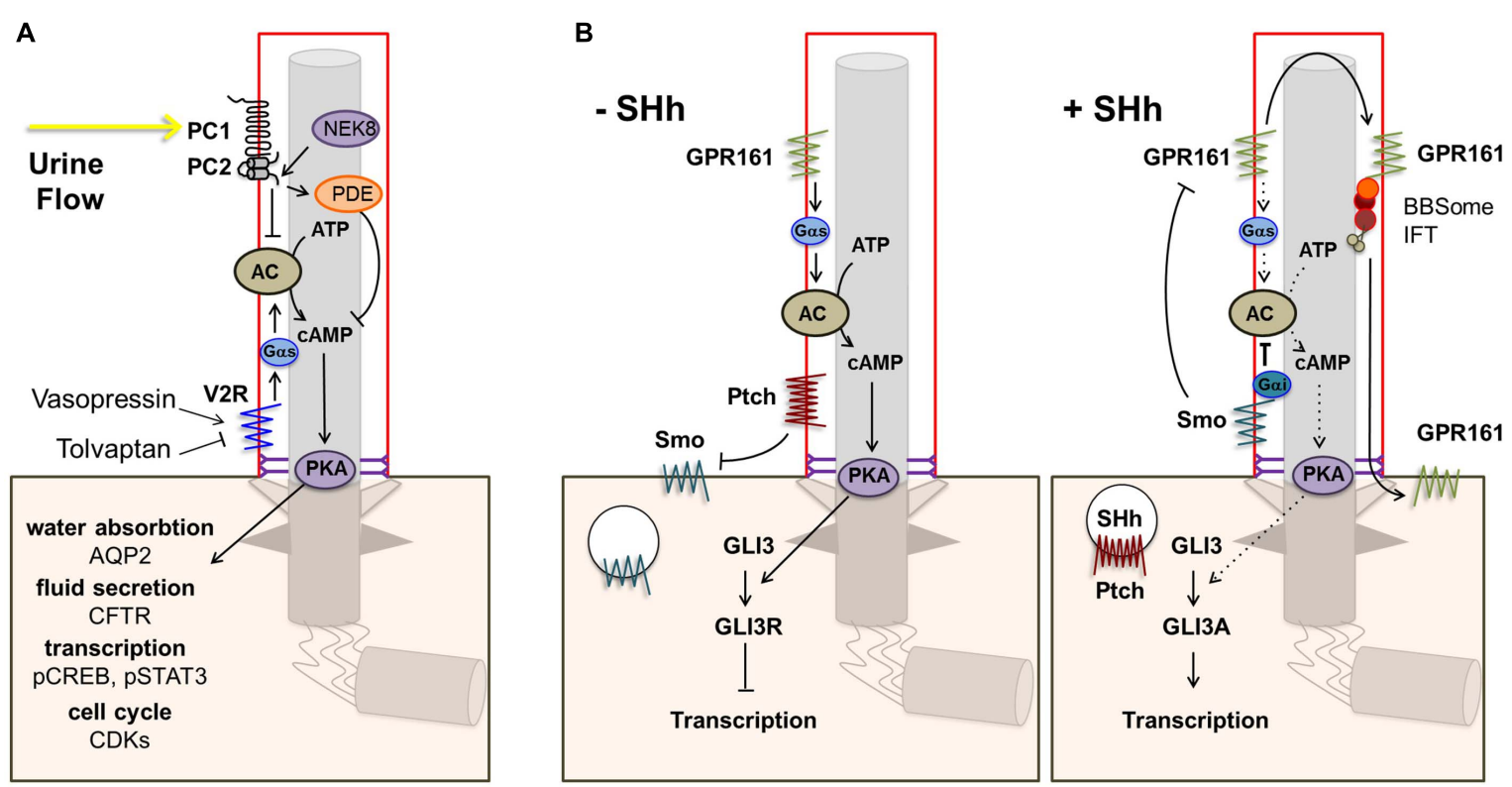

FIGURE 2 | Ciliary GPCR signaling. (A) The vasopressin receptor type 2 (V2R) is located in the ciliary membrane. When activated, signal transduction via Gs alpha subunit (Gas) leads to increased production of cAMP by adenylate cyclase (AC) and subsequent activation of protein kinase A (PKA). PKA phosphorylates aquaporin 2 (AQP2), which translocates to the apical cell membrane leading to increased water reabsorption. In addition, PKA phosphorylates the cystic fibrosis transmembrane conductance regulator (CFTR), leading to transport of $\mathrm{Cl}^{-}$ions and fluid secretion into the lumen, and CREB and STAT3, enhancing transcription. Other downstream effects include increased CDK signaling. Opening of the polycystin-1/polycystin-2 (PC1/PC2) complex in response to urine flow inhibits the CAMP/PKA pathway via inhibition of AC5/6 and through PDE4C. (B) Other main ciliary GPCRs are Smo and the constitutively active Shh receptor GPR161. In the absence of Shh, Ptch1 excludes and inhibits Smo, leading to repression of Gli transcription factors by SuFu, and GPR161 signaling leads to increased cAMP-dependent activation of PKA. Elevated PKA further represses Gli transcription factors. Binding of a Hh ligand leads to entry of Smo and consequent removal of GPR161 from the ciliary membrane, resulting in decreased cAMP/PKA activity and activation of Gli transcription factors.

drug registered for treatment of adult ADPKD patients below 55 years of age and at risk of rapidly progressive disease as defined by clinical and/or genetic criteria (Chebib et al., 2018). There are no clinical trials registered for use of Tolvaptan in patients with NPH and there is only one case report in which Tolvaptan is described for the treatment of NPHP3-related infantile NPH without an effect on the very rapid renal function decline in this specific severe case (Strong et al., 2018). Such an approach could theoretically be used in the case of cystic infantile NPH, although the therapeutic window is small (mean age under 5), while its relevance in juvenile NPH is less apparent. Indeed, the presence of cysts is a late event in the juvenile/adult form and the potential role of cAMP remains to be investigated.

Another class of drugs that modulate renal cAMP levels by binding to GPCRs are somatostatin analogs, which activate somatostatin receptors and inhibit AC through the inhibitory G protein Gai (Günther et al., 2018). Somatostatin analogs such as octreotide are used in the treatment of neuroendocrine tumors as inhibitors of growth hormone and several pancreatic and gastrointestinal hormones. In the context of kidney disease, octreotide was first shown to be well-tolerated and slow renal volume expansion in a 6-month trial with 14 adult patients with ADPKD (Ruggenenti et al., 2005). Octreotide was later shown to inhibit hepatic cyst development in the PCK rat model and reduce liver volume in post hoc analysis of the abovementioned study in ADPKD patients (Masyuk et al., 2007;
Caroli et al., 2010). It also reduced kidney volume in patients with ADPKD compared to placebo-treated patients (Hogan et al., 2010). In the context of NPH, octreotide was shown to significantly decrease cAMP levels and to rescue cilia defects and abnormal 3D spheroid phenotypes in Nphp3, Cep290/Nphp6 and Rpgrip1l/Nphp8 knockdown IMCD3 cells (Ghosh et al., 2012). These results suggest a positive effect of treatment with somatostatin analogs in NPH, although results have not been replicated in an animal model. Similarly, targeting the calciumsensing receptor (CasR), a GPCR expressed in renal tubular cells, also leads to decreased production of cAMP through inhibition of AC (Riccardi and Valenti, 2016). Administration of the calcimimetic R-568 CasR agonist was shown to decrease cystogenesis and renal fibrosis in $p c y / N p h p 3$ mice (Chen et al., 2011). The above examples clearly show that decreasing cAMP levels is an effective therapeutic option in models of cystic kidney disease including ADPKD and (mostly infantile) NPH. The relevance of targeting cAMP in the context of juvenile NPH remains an open question as cystogenesis through proliferation and fluid secretion could be a less critical mechanism in this form of NPH (Srivastava et al., 2018).

A class of bioactive lipid metabolites called cyclooxygenase (COX) oxylipins (e.g., prostaglandins, leukotrienes) has been shown to be elevated in diseased kidneys of rodent models of NPH ( $p c y / N p h p 3$ and jck/Nek8/Nphp9 mice and Han:SPRDCy/Anks6/Nphp16 rats) and other cystic kidney diseases 
(Ibrahim et al., 2014; Yamaguchi et al., 2014; Monirujjaman et al., 2017). A flax oil-enriched diet effectively reduced levels of COX-derived oxylipins compared to a control diet and mitigated interstitial fibrosis and cyst growth in $p c y / N p h p 3$ mice (Sankaran et al., 2004; Yamaguchi et al., 2015). In addition, treatment with COX inhibitors or dietary soy protein slowed disease progression in a heterozygous Han:SPRD-Cy/Anks6/Nphp16 rat model (Sankaran et al., 2007; Ibrahim et al., 2014, 2015). Interestingly, the COX inhibitor Ibuprofen is used to treat patients presenting with Bartter Syndrome which is characterized by water and salt loss associated with elevated urinary prostaglandin E2 (PGE2). However, treatment of PCK rats with a COX inhibitor did not show any improvement of the cystic index or of kidney function (Sussman et al., 2020).

Although the exact role of COX-derived oxylipins in CKD remains unclear, PGE2 is known to exert its effect partly through increasing levels of cAMP through activation of tubular EP2/4 receptors (Elberg et al., 2007; Monirujjaman et al., 2017). The GPCR EP4 is one of the PGE2 receptors which control water and salt reabsorption (Li et al., 2018). Interestingly, EP4, which is also involved in AQP2 trafficking and could functionally compensate for the loss of V2R ( $\mathrm{Li}$ et al., 2009), is present at cilia where it is involved in CAMP production and positive regulation of ciliogenesis through positive regulation of IFT (Jin et al., 2014; Jiang et al., 2019). It has however to be noticed that PGE2 acts through activation of four distinct receptors (EP1-4, see above) which control opposite downstream signaling pathways, complicating the understanding of the role of PGE2 in renal ciliopathies. Indeed, a recent study indicate that while PGE2 is pro-cystogenic in vitro, inhibition of EP2 and EP4 with antagonists resulted in a more severe disease in a $P k d 1$ model due to increased inflammation (Lannoy et al., 2020). Even if among oxylipins PGE2 is an interesting candidate in the context of NPH (see above), evidence for increased or decreased production of PGE2, or of any other COX-derived oxylipins, in NPH patients is lacking so far.

\section{mTOR inhibition}

Next to cAMP signaling, an important signaling pathway that is inappropriately activated in ciliopathies is the mammalian target of rapamycin (mTOR) which functions as a central regulator in cellular metabolism, growth, proliferation, cell cycle and survival. It has been shown that cilia-dependent flow sensing negatively regulates mTORC1 through the kinase LKB1 (Boehlke et al., 2010). In ciliopathies like PKD, flow sensing is altered leading to mTOR pathway activation and uncontrolled cell growth and proliferation which induces cystogenesis (Lai and Jiang, 2020).

Administration of the mTOR pathway inhibitor rapamycin was shown to decrease proliferation, cystogenesis, kidney enlargement and renal function decline in heterozygous Han:SPRD-Cy/Anks6/Nphp16 rats (Tao et al., 2005). In addition, ADPKD patients who had received kidney transplants were observed to have reduced volumes of affected kidney and liver if an mTOR inhibitor was included in the immunosuppressive regimen (Shillingford et al., 2006; Qian et al., 2008). However, 18-month rapamycin treatment of adults with early stage ADPKD did not halt disease progression and a clinically effective dose could not be achieved without systemic adverse effects
(Serra et al., 2010; Shillingford et al., 2012). A study in zebrafish with morpholino-based knockdown of ciliopathy-associated genes including $n p h p 2, n p h p 5, n p h p 6$ and $n p h p 11$ provided additional evidence for the potential efficacy of mTOR inhibition in NPH-related ciliopathies. Treatment with rapamycin restored renal size and morphology in all morphants and partially rescued renal filtration decrease (Tobin and Beales, 2008). Treatment of $p c y / N p h p 3$ mice with rapamycin did not prevent initial cyst development but significantly reduced cyst enlargement, fibrosis and renal insufficiency (Gattone et al., 2009). This suggests an application in late-stage progression of NPH, the stage in which $\mathrm{NPH}$ is typically detected, if future targeted delivery can prevent systemic side effects of rapamycin.

\section{Modulators of Hedgehog signaling}

The ciliary GPCRs SMO and GPR161 are key players in the Hh signaling pathway, which is essential in embryonic development and adult tissue homeostasis and regeneration (Kopinke et al., 2020). In the absence of $\mathrm{Hh}$, patched (PTCH1) localizes to the ciliary membrane and excludes and inhibits SMO. In addition, constitutive GPR161 activity in the cilium stimulates AC leading to increased cAMP-dependent activation of PKA which further leads to repressor forms of the GLI3 transcription factor (GLI3R). Upon binding of Hh to PTCH1, SMO enters the cilium whereas GPR161 is removed from the ciliary membrane, leading to decreased cAMP/PKA activity and activation of GLI3 (GLI3A; Figure 2B).

Hh signaling is involved in kidney development and preservation of the differentiated state of renal tubular epithelial cells (Li et al., 2011). Interestingly, Hh signaling has been implicated in fibrosis in different tissues through involvement in proliferation of myofibroblasts which expand in fibrotic tissues and secrete ECM components (Distler et al., 2019). In the kidney, Hh was shown to be produced in high amount by tubular cells in different models of kidney injury, stimulating interstitial mesenchymal cells which leads to fibrosis (Tan et al., 2016). In contrast, activation or downregulation of Hh signaling did not affect cyst formation in a $P k d 1$ mouse model (Ma et al., 2019). NPHP proteins were shown to play important roles in $\mathrm{Hh}$ signaling including RPGRIP1L and ZNF423 as well as IFT involved in the transport of GPR161 and GLI transcription factors in and out of cilia (Vierkotten et al., 2007; Qin et al., 2011; Hong and Hamilton, 2016). Indeed, the Hh pathway has been shown to be dysregulated in numerous ciliopathies (Ruiz-Perez et al., 2007; Aguilar et al., 2012). Of note, a CRISPR-based screen to identify regulators of $\mathrm{Hh}$ signaling detected among many ciliopathy genes few genes mutated exclusively in $\mathrm{NPH}$, not including NPHP1 and NPHP4 or the genes encoding InvsC proteins, suggesting that disrupted $\mathrm{Hh}$ signaling plays a less prominent role in isolated $\mathrm{NPH}$ than in other ciliopathies (Breslow et al., 2018).

A Cep290-gene trap mouse model of Joubert syndrome revealed increased Gli3 repressor isoform in the kidney, in agreement with decreased Hh signaling. Modulation of this pathway with the $\mathrm{Hh}$ agonist purmorphamine reconstituted impaired spheroid formation in collecting duct cells from Cep290-mutant mice and in URECs derived from a patient 
with Joubert syndrome (Hynes et al., 2014) as well the elongated cilia phenotype (Srivastava et al., 2017). In contrast, inhibition of Hh signaling using GLI and SMO small molecule antagonists Gant61 and Sant2 respectively prevented cyst formation in kidney explants from Ttc21b/Ift139-conditional knockout, $j c k / N e k 8 / N p h p 9$ and $P k d 1$ mutant mice (Tran et al., 2014). In contrast to CEP290, TTC21B/IFT139 is a negative regulator of Hh signaling (Tran et al., 2008), similar to other IFT-A subunits which control ciliary transport of GPR161 (Kopinke et al., 2020). The impact of ciliopathies on $\mathrm{Hh}$ signaling is therefore complex and appears to be gene and tissue dependent and may rely on its impact on the level of ciliary cAMP production. Although in vitro modulation of $\mathrm{Hh}$ signaling yielded promising results, pharmacological studies in patients are unlikely to be executed because of the risk of serious side effects including medulloblastoma associated with Hh stimulation (Molinari and Sayer, 2017).

\section{YAP inhibition}

The Hippo pathway controls numerous biological processes and is an important modulator of cell proliferation and organ size (Müller and Schermer, 2020). NPHP4 represses the Hippo signaling pathways through inhibition of LATS1/2-mediated phosphorylation of YAP and TAZ transcription factors, thereby stimulating transcription of target genes and cell proliferation (Habbig et al., 2011). In addition, InvsC proteins including NEK8/NPHP9 and NPHP3 form a complex that controls nuclear translocation of YAP/TAZ and transcription of target genes (Habbig et al., 2012; Frank et al., 2013). Dysregulation of the Hippo pathway can cause antiproliferative signaling resulting in hypodysplastic phenotypes and loss of differentiation, or increased proliferation leading to cystic phenotypes depending on the type of mutation (Habbig et al., 2011; Grampa et al., 2016; Müller and Schermer, 2019; Xu et al., 2020).

Transient Nek8 knockdown in mIMCD3 cells resulted in increased YAP activation and enlarged spheroids, which could be rescued by administration of Verteporfin, an inhibitor of YAP transcriptional activity. Verteporfin additionally rescued pronephric cysts in zebrafish embryos overexpressing human NEK8 (Grampa et al., 2016). Verteporfin also reduced fibrosis in the unilateral ureteral obstruction mouse model (Szeto et al., 2016). Interestingly, Hippo components Mst1/Mst2 double knockout mice presented with an NPH-resembling phenotype comprising fibrosis, tubular dilation and thickening of tubular basement membranes. Additional knockout of YAP rescued this kidney phenotype (Xu et al., 2020), further indicating that targeting of the Hippo pathway could be an interesting approach for NPH.

\section{Wnt inhibition}

Another key developmental pathway is the Wingless-Int-1 (Wnt) pathway, although the role of cilia in canonical, $\beta$-catenindependent Wnt signaling remains controversial (Wheway et al., 2018). Proteins from the InvsC disrupted in infantile $\mathrm{NPH}$ including INVS/NPHP2 and NPHP3 have been proposed to be required for the switch from canonical Wnt signaling, involved in cell proliferation, to non-canonical Wnt signaling which maintains planar cell polarity, although the precise role in cystogenesis is debated (Simons et al., 2005; Bergmann et al., 2008; Sugiyama et al., 2011; Wang et al., 2018). Planar cell polarity is essential for oriented cell division which controls tubule elongation and is impaired in most murine renal ciliopathy models (Fischer and Pontoglio, 2009). Interestingly, RPGRIP1L and NPHP4 were also involved in Wnt signaling through their functional interaction with INVS (Burckle et al., 2011; Mahuzier et al., 2012).

Increased canonical Wnt signaling has been suggested to induce cystogenesis in PKD and NPH (Malik et al., 2020). Knockdown of DCDC2/NPHP19 has been shown to activate the canonical Wnt pathway and cause pronephric cyst formation in zebrafish, a phenotype which could be rescued by a $\beta$-catenin inhibitor (Schueler et al., 2015).

\section{Targeting Cilia-Independent Signaling Pathways and Cell Cycle Regulation CDKs}

Specific ciliary proteins are involved in DDR signaling and consequent cell cycle regulation. Although drugs that interfere directly with DDR signaling have not been studied in this context, the dysregulated cell cycle has been investigated as a target for the treatment of cysts and fibrosis in renal ciliopathies (IbraghimovBeskrovnaya, 2007; Choi et al., 2013).

Administration of the broad $\mathrm{CDK}$ inhibitor roscovitine (which targets CDK1, CDK2, CDK5 and CDK7, arresting the cell cycle) effectively arrested kidney volume expansion, cyst progression and renal insufficiency in jck/Nek8/Nphp9 and $c p k$ (cystin) mouse models of infantile NPH and PKD, respectively, with long-lasting effects. Analysis of the targets of roscovitine revealed induction of a G1/S cell-cycle block, transcriptional regulation and inhibition of apoptosis as mechanisms of action (Bukanov et al., 2006). In addition to the mouse models, roscovitine treatment also showed partial rescue of pronephric cysts and improved renal filtration capacity in zebrafish morphants of ciliopathy-associated genes (Tobin and Beales, 2008). Another study in jck/Nek8/Nphp9 mice demonstrated that roscovitine ameliorated the elongated cilia phenotype and restored tubular epithelial differentiation, while conditional inactivation of CDK5 reduced cilia length, total kidney volume and cyst formation (Husson et al., 2016). In contrast, Cdk2-deficient jck/Nek8/Nphp9 mice showed no improvement of renal cyst growth, inflammation and fibrosis, likely due to compensatory upregulation of $C d k 1$. In addition, treatment with the mTOR inhibitor rapamycin reduced Cdk1 and Cdk2 activity and attenuated the cystic phenotype, suggesting that the effect of mTOR inhibition is partly mediated by CDK inhibition (Zhang et al., 2020). Finally, roscovitine was demonstrated to attenuate renal cyst progression in a kidneyspecific Cep164-knockout mouse model characterized by rapidly progressive cystic kidney enlargement (Airik et al., 2019). In summary, studies performed mostly in models of infantile NPH have demonstrated that targeting cell cycle dysregulation and proliferation using CDK inhibitors is an efficient approach to limit cyst growth. Of note, part of the effects of roscovitine, and also of its derivative CR8, could however be linked to its potent 
inhibition of Casein Kinase 1 family members which expression pattern is perturbed in cystic mouse model including infantile NPH models ( $p c y / N p h p 3, J c k / N e k 8 / N p h p 9)$ (Billot et al., 2018). Interestingly, this study also indicates that the expression of the cell cycle regulator p21cip1/WAF1, which inhibits CDK2, is decreased in all cystic models while it is increased in the kidneys of Nphp4 mutant mice, again suggesting differential pathophysiological mechanisms between cystic infantile and juvenile forms of NPH.

Interestingly, targeting of CDK1/2 was also shown to be effective in a Cep290/Nphp6 mouse model characterized by slowly progressive kidney disease. Treatment of kidney cells from Cep290-deficient mice with CDK1/2 inhibitor rescued DNAdamage signaling, supernumerary centrioles and ciliation defects (Slaats et al., 2015). Furthermore, knockdown of CDK5 in URECs from a patient with Joubert syndrome with compound heterozygous mutations in CEP290 mitigated the elongated cilia phenotype observed in these cells. In addition, treatment of the cells with the Hh agonist purmorphamine reduced expression of CDK5, suggesting a convergence of the Hh and CDK signaling pathways (Srivastava et al., 2017).

\section{Calmodulin and $\mathrm{Ca}^{2+} /$ calmodulin-dependent protein kinase II}

$\mathrm{Ca}^{2+} /$ calmodulin-dependent protein kinase II (CaMKII) is an important mediator of the endoplasmic reticulum stress response, oxidative stress and the mitochondrial apoptotic pathway, which were shown to be upregulated in kidneys from jck/Nek8/Nphp9 mice. Pharmacological inhibition of CaMKII restored these pathways and significantly reduced kidney volume and cystogenesis (Bracken et al., 2016). CaMKII additionally has a role in cell cycle progression (Skelding et al., 2011). CaMKII inhibition could therefore also reduce cystogenesis through a mechanism similar to CDK inhibitors. Interestingly, calmodulin is known to bind to IQCB1/NPHP5 (Otto et al., 2005) and was recently shown to negatively regulate the amount of NPHP5 present at the transition zone (Kim et al., 2018). Eupatilin, a compound able to rescue ciliogenesis in CEP290 invalidated cells (see below), directly modulates the calmodulin/NPHP5 interaction therefore increasing the amount of NPHP5 at the transition zone in the absence of CEP90 (Kim et al., 2018). Interestingly, Eupatilin was recently shown to improve cilia-related phenotypes in the context of RPGRIP1L/NPHP8 suggesting that it might present broader potential in transition zone-related NPH (Wiegering et al., 2021).

\section{ERK and p38-MAPK inhibition}

The ERK pathway (downstream of receptor tyrosine kinases and GPCR signaling), is involved in regulation of cell cycle and proliferation and was shown to be activated as a consequence of increased cAMP in ADPKD (Yamaguchi et al., 2003). Inhibition of ERK activation slowed down disease progression in $p c y / N p h p 3$ mice (Omori et al., 2006). It also resulted in decreased fibrosis and cystogenesis in an Invs/Nphp2 mouse model (Okumura et al., 2009). The same group later showed that a p38 mitogen-activated protein kinase (MAPK) inhibitor reduced renal fibrosis but not cystogenesis independently of ERK activation, however, this did not improve kidney function and survival rate of Invs mice (Sugiyama et al., 2012).

\section{Inflammation and Senescence}

In addition to ciliary signaling pathways and cystogenesis, specific interventions directly target development of tubulointerstitial fibrosis, a hallmark histopathological feature of juvenile and adult NPH. The role of inflammation in fibrosis has been discussed in section "Inflammation and Senescence." Several groups have targeted the immune response in NPH. For example, rodent models of infantile NPH (Han:SPRD$C y / A n k s 6 / N p h p 16$ rats and $p c y / n p h p 3$ mice) treated with the anti-inflammatory drug methylprednisolone showed reduced fibrosis and preserved kidney function compared to untreated animals (Gattone et al., 1995). Treatment of Han:SPRDCy/Anks6/Nphp16 rats with the anti-inflammatory drug resveratrol attenuated cyst formation, elevated serum creatinine and macrophage infiltration partly through inhibition of mTOR signaling and the NF-kB pathway (Wu et al., 2016). Furthermore, a study using LPK/Nek8/Nphp9 mutant rats found that treatment with the immunomodulatory drug dimethyl fumarate, which activates the Nrf2 pathway and inhibits NF-kB signaling, reduced macrophage infiltration but did not ameliorate cyst progression or renal insufficiency (Oey et al., 2018).

Replicative senescence in tubular cells, which leads to secretion of pro-inflammatory molecules, has been shown to cause extensive kidney damage in Glis2/Nphp7 knockout mice (Jin et al., 2020) (see section "Pathophysiology of Nephronophthisis"). The senolytic (anti-aging) drug forkhead box protein $\mathrm{O} 4 \mathrm{D}$-retro inverso (FOXO4-DRI), which induces apoptosis of senescent cells through inhibition of FOXO4/P53 interaction (Baar et al., 2017), effectively eliminated senescent cells in mouse models for acute kidney disease and reduced inflammation and tubulointerstitial fibrosis while preserving renal function in Glis2-knockout mice (Jin et al., 2019, 2020). The role of senescence and the efficacy of senolytic drugs in other types of NPH is unknown and should be investigated further.

\section{Glycosphingolipids and Cholesterol}

Several studies demonstrated that glycosphingolipid metabolism is perturbed in PKD (Deshmukh et al., 1994; Chatterjee et al., 1996). Interestingly, glycosphingolipids are components of rafts, membrane microdomains involved in the assembly of signaling platforms at the plasma membrane that are also present at the ciliary membrane. Dysregulated production of those lipids may therefore contribute to perturbed signaling and therefore to cystogenesis (Natoli et al., 2020). Interestingly, glucosylceramides, which are glycosphingolipid precursors, were found to be highly increased in the kidneys of cystic mouse models including in $p c y / N p h p 3$ and Jck/Nek8/Nphp9 mice and pharmacological or genetic inhibition of glucosylceramide synthase activity efficiently reduced renal cysts in Jck/Nek8/Nphp9 mice (Natoli et al., 2010, 2012).

Besides glycosphingolipids, cholesterol is also involved in many cellular functions including in signaling through Ras and plasma membrane lipid rafts (Ahmadi et al., 2020). Cholesterol synthesis can be modulated by statins, a family of molecules 
which have been widely used to treat hypercholesterolemia. In addition to its classical well-established functions, cholesterol is enriched in the ciliary membrane and was revealed to play an important role in ciliogenesis (Maerz et al., 2019) as well as in $\mathrm{Hh}$ signaling as a direct modulator of SMO activity (Kong et al., 2019). Interestingly, and in agreement with those observations, mutations in genes encoding actors of the cholesterol biosynthesis pathway were associated with skeletal disorders (Suzuki et al., 2020) resembling those associated with mutations in IFT-A subunit encoding genes (see introduction). Statins were shown to improve CKD in various animal models (Ecder, 2016) and were also shown to improve renal function in the Cy/Anks6/Nphp16 rat model, likely through an effect on the renin-angiotensin system (Gile et al., 1995; Zafar et al., 2007). Several studies obtained conflicting results on the beneficial effects of statins in PKD patients (Fassett et al., 2010; Cadnapaphornchai et al., 2014; Brosnahan et al., 2017; Müller and Schermer, 2019). It is currently not known if cholesterol dysregulation is associated with NPH or if modulating cholesterol synthesis could be an interesting approach in the context of $\mathrm{NPH}$ other than in infantile NPH rodent models.

\section{Microtubule Dynamics}

Cytoplasmic microtubule dynamics directly influences ciliogenesis (Sharma et al., 2011) and their reorganization tightly controls polarity establishment in epithelia (Toya and Takeichi, 2016). Interestingly, ciliopathy conditions were associated with increased stability of cytoplasmic microtubules (Berbari et al., 2013), a phenotype which was more deeply investigated in the context of NPH and TRAF3IP1/IFT54 mutant conditions (Berbari et al., 2011; Bizet et al., 2015). Impaired IFT54 function led to overexpression of the microtubule associated protein MAP4 and lowering MAP4 expression rescued polarity defects in vitro and partially rescued ciliopathy phenotypes in zebrafish morphants (Bizet et al., 2015). It would be interesting to investigate if stabilization of the microtubules is a phenotype shared with other NPHP defects. These results indicate that modulators of cytoplasmic microtubule dynamics could be a potential therapeutic approach for NPH.

Interestingly, expression of HDAC6 was found to be increased in various kidney conditions including fibrotic, ADPKD and CKD models, and HDAC6 inhibitors showed a positive effect on disease evolution (Ke et al., 2018). HDAC6 is a histone deacetylase which is key regulator of the cytoskeleton through deacetylation of $\alpha$-tubulin and cortactin. HADC6 was also found to be involved in cilia disassembly through destabilization of axonemal microtubules (Pugacheva et al., 2007; Loktev et al., 2008). Functions of HDAC6 are wide and complex, however, and its overexpression likely yields deacetylation which is expected to result in destabilization of microtubules (contrary to increased stability of microtubules observed in TRAF3IP1/IFT54 mutant conditions). This suggests differential microtubule involvement between NPH and other renal disorders.

\section{Drug Screens for Nephronophthisis}

Pharmacological screens for ciliopathies comprise small molecule compound screens and high-throughput testing of chemical libraries such as United States Food and Drug Administration (FDA)-approved drugs with the aim of drug repurposing. The latter is an attractive option because it is cheaper, faster, and has a higher success rate than development of novel drugs (Forsythe et al., 2018). Thus far, compound screens in cystic renal disease have mostly been performed for ADPKD, the most common ciliopathy affecting the kidney (Booij et al., 2017, 2019). High-throughput drug screens for NPH-related ciliopathies are scarce in the literature and findings have not been replicated (Kim and Kim, 2019).

One of the few published compound screens was conducted by Kim et al. (2018), who screened a library of 2,789 synthetic and natural compounds in CEP290-invalidated human RPE1 cells and identified 22 compounds that restored ciliogenesis, of which the flavonoid eupatilin showed the strongest effect. Eupatilin was demonstrated to restore IQCB1/NPHP5 levels at the ciliary transition zone in the absence of CEP290 (see above). Eupatilin treatment did not significantly rescue ciliogenesis defects associated with the knockdown of other NPH-associated genes, including NPHP4, CEP164/NPHP15 and CEP83/NPHP18, suggesting a CEP290-specific effect. Treatment of rd16 mice harboring a Cep290 in-frame deletion with eupatilin improved cone photoreceptor function, showing its efficacy in vivo (Kim et al., 2018). However, because this Cep290 mouse model of Leber congenital amaurosis (LCA) does not present a kidney phenotype (Chang et al., 2006), the effect of eupatilin on NPH was not investigated.

Two compound screens were performed in zebrafish models. A library of 115 compounds targeting cilia specific pathways was screened in $p k d 2$ - and ift172-morphant embryos which present pronephric cysts (Cao et al., 2009). A histone deacetylase (HDAC) inhibitor was identified that suppressed cyst formation in pkd2- but not in ift172-morphants, making this finding less relevant for NPH. Cox2-inhibitors and a CaMKII inhibitor were identified to affect extrarenal ciliopathy phenotypes in both models (Cao et al., 2009). In addition, an automated imaging pipeline was recently developed to efficiently profile a large compound library in ift172-morphant zebrafish embryos, however, identified pronephric cyst-modifying compounds were not reported (Pandey et al., 2019).

Another strategy that has been employed is an untargeted small interfering RNA (siRNA) screen to identify genes that modulate ciliogenesis and can be targeted pharmacologically. For example, an siRNA screen in RPE1 cells targeting 7,784 therapeutically relevant genes identified 36 positive modulators of ciliogenesis including proteins involved in the regulation of actin cytoskeleton dynamics. Pharmacological inhibition of actin polymerization using cytochalasin D rescued ciliogenesis in Ift88mutant mouse embryonic fibroblasts (Kim et al., 2010). However, cytochalasin D is likely not specific enough for treatment of ciliopathies. In addition, a recent genome-wide siRNA screen identified 10 genes that rescued aberrant Wnt signaling in BBS4depleted cells (Tsai et al., 2019). One of the identified genes encoded USP35, a negative regulator of the ubiquitin proteasome system. Suppression of USP35 improved the kidney phenotype in bbs4-morphant zebrafish embryos, suggesting that impaired clearance of signaling molecules could play a role in NPH 
pathophysiology. Next, a small molecule inhibitor of another deubiquitinase (USP14; IU1) was tested in zebrafish and did not have a significant effect on the kidney phenotype, consistent with the results from the screen (Tsai et al., 2019). High-throughput phenotype-based screening methods using zebrafish and cell models have been outlined for renal ciliopathies (Gehrig et al., 2018; Pandey et al., 2019; Zhang P. et al., 2019).

\section{GENE-BASED THERAPY}

A promising gene-based strategy makes use of antisenseoligonucleotides (ASO) to modulate splicing. This approach was first employed to correct a recurrent intronic CEP290/NPHP6 mutation present in $10-15 \%$ of LCA cases, which introduces a cryptic splice site leading to inclusion of a pseudo exon with a premature stop codon (Collin et al., 2012). ASO targeting of this mutation successfully restored mRNA splicing, CEP290 expression and cilia in patient cells and patient iPSC-derived retinal organoids (Gerard et al., 2012; Parfitt et al., 2016) and led to vision improvement in LCA patients (Cideciyan et al., 2019). ASO was also used to induce skipping of nonsense mutationbearing exons as well as treat non-retinal manifestations. For example, systemic ASO treatment of a Cep290 mouse model of Joubert syndrome resulted in reduced kidney cyst burden compared to untreated mice (Ramsbottom et al., 2018). While CEP290 is unique in that it contains numerous in-frame exons that can be targeted by ASO, basal exon skipping has also been shown to modify the phenotype in CC2D $2 A$-related Joubert syndrome and could be induced in other ciliopathy-associated genes (Drivas et al., 2015; Ramsbottom et al., 2018). Although ASO cannot be applied in case of a homozygous NPHP1 deletion which is the most common cause of NPH, it can be used to target downstream dysregulated genes as was demonstrated for Agt encoding angiotensinogen in the treatment of $P k d 1$ mice (Saigusa et al., 2016; Fitzgibbon et al., 2018). An advantage of ASO-based treatment for NPH is that circulating ASO are cleared by and accumulate in the kidney where they are taken up especially but not exclusively by tubular cells, facilitating delivery to target tissue (Oberbauer et al., 1996; Lakhia et al., 2019). Research into ligandbased targeted ASO delivery is ongoing (Kim and Kim, 2019).

Additionally, CRISPR/Cas9 has shown promising results for the treatment of NPH in in vitro systems. In contrast to classical gene therapy in which a wild-type copy of the gene is integrated into the host genome, CRISPR/Cas9 is a precise genome-editing technique that allows use of a repair template. This correction approach is widely used to obtain isogenic control iPSCs and it was shown to efficiently restore the cilia phenotype in iPSC-derived kidney organoids from a patient with IFT140-related Mainzer-Saldino syndrome. In addition, spheroids from epithelial cells sorted from uncorrected organoids were less polarized than spheroids from gene-corrected cells (Forbes et al., 2018). However, implementation in patients with renal ciliopathies is met by technical challenges (for example off-target effects, delivery to the kidney and evading an immune response) and ethical obstacles. In addition, the extreme genetic heterogeneity in NPH-related ciliopathies, i.e., the absence of mutation hot spots except for the homozygous NPHP1 deletion, and possible role of modifier variants, limit the practical and financial feasibility of gene-targeted therapies (Kim and Kim, 2019).

\section{DEVELOPMENTS AND FUTURE DIRECTIONS}

Nephronophthisis is an important hereditary cause of ESRD in children and young adults for which there is currently no targeted therapy. Pharmacological interventions aimed at restoring aberrant signal transduction (for example V2R antagonists) or dysregulated cell cycle (for example CDK inhibitors) as well as the use of immunomodulatory drugs (for example senolytic drugs) have improved cystic phenotypes in zebrafish and rodent models of NPH (Gattone et al., 2003; Bukanov et al., 2006; Jin et al., 2020). In addition, ASO therapy partly restored gene expression and reversed cyst burden in a Cep290 mouse model of Joubert syndrome (Ramsbottom et al., 2018). ASO therapy has been approved by the FDA for clinical use in several human diseases including spinal muscular atrophy and Duchenne muscular dystrophy, suggesting that application in $\mathrm{NPH}$ is feasible (Fay et al., 2020). However, the Cep290 mouse model, like most rodent and zebrafish models reviewed here, does not exhibit the massive tubulointerstitial fibrosis observed in NPH patients, which severely limits conclusions about efficacy of potential therapy for juvenile NPH (Hynes et al., 2014; Ramsbottom et al., 2018).

Truly representative models for juvenile NPH are not available yet except for Glis2/Nphp7 knockout mice, and GLIS2 mutations are an atypical and rare cause of NPH (Lu et al., 2016). In fact, most mouse models used in research on NPH therapy correspond genetically and phenotypically mainly to infantile $\mathrm{NPH}$, which represents a minor fraction of NPH patients, is histologically different from the more prevalent juvenile NPH and shows a more rapid disease course (see introduction).

Alternative non-orthologous animal models that mimic juvenile/adult NPH could also be explored. For example, mice with kidney-specific knockout of $L k b 1$ showed a urine concentration defect, diminished kidney size, cysts at the corticomedullary junction, tubular basement membrane thickening, inflammation and fibrosis (Han et al., 2016; Viau et al., 2018). Interestingly, LKB1 (STK11) was shown to be part of a ciliary module comprising NPHP1, NEK7, ANKS3 and polycystin-1 that regulates cilia-controlled secretion of CCL2, a chemokine that promotes macrophage recruitment and consequent cyst growth and interstitial inflammation (Viau et al., 2018). In addition, mice with kidney-specific knockout of Prkarla, a regulatory subunit of PKA, presented with normalsized kidneys with small cysts and interstitial fibrosis (Ye et al., 2017), suggesting that cAMP signaling defects not only play a role in cystogenesis but also in the development of juvenile NPHspecific phenotypes. Kidney-specific knockout of Aatf (DNA damage response target Apoptosis Antagonizing Transcription Factor), encoding a regulator of p53 during DDR, also leads to phenotypes similar to juvenile NPH including tubular atrophy, 
interstitial fibrosis and cysts at the corticomedullary junction (Jain et al., 2019). Lastly, Fan1 knockout mice treated with cisplatin showed a histological phenotype that included tubular dilations, tubular basement membrane thickening and interstitial fibrosis, resembling juvenile NPH except for the presence of karyomegalic nuclei (Zhou et al., 2012; Airik et al., 2016). These models might point to key signaling pathways and might prove more useful for testing fibrosis-modulating drugs for juvenile $\mathrm{NPH}$ than genetically identical models.

During the past 5 years, an increasing number of pharmacological studies in NPH have focused on fibrosisassociated pathways and fibrosis-modulating agents. Drugs that interfere with cell cycle progression such as CDK inhibitors typically affect ubiquitous and essential cellular processes, which limits their application in NPH treatment. Alternatively, the pro-inflammatory response downstream of cell cycle arrest can be targeted to prevent tubular damage and fibrosis (Jin et al., 2020). Specifically, the role of cellular senescence and efficacy of senolytic drugs remains to be investigated in prevalent $\mathrm{NPH}$ subtypes. Of note, the CDK inhibitor roscovitine was shown to promote apoptosis of neutrophils and enhance resolution of inflammation, indicating crosstalk between both mechanisms (Rossi et al., 2006). Additional progress can be gained from repurposing of medication approved for other fibrotic kidney diseases, for example COX inhibitors which have shown potential in the prevention of diabetic nephropathy in addition to ameliorating NPH in rodent models (Goru, 2018).

Despite in vitro and in vivo evidence for drugs that ameliorate (part of) the NPH phenotype, there is a striking lack of clinical trials for NPH. To exclude possible publication bias, ClinicalTrials.gov was queried for registered ongoing and future clinical trials in ciliopathies. This yielded trials for ADPKD, ARPKD, primary ciliary dyskinesia, Von Hippel Lindau disease and various extrarenal manifestations of ciliopathies, including obesity in Bardet-Biedl and Alström syndromes (clinicaltrials.gov - access date 2021 April 12). There are several possible reasons for the absence of clinical trials in NPH, including challenges related to the genetic and phenotypic heterogeneity in NPH, for example small sample sizes and variability within cohorts, the relatively short therapeutic window compared to ADPKD, challenges of clinical trials in a pediatric population and financial aspects of orphan drug development.

In light of this, research groups should capitalize on available data in renal ciliopathies and investigate associations between medication for (extra)renal symptoms and fluctuation in renal phenotype, for example statins which can be prescribed to treat hypercholesterolemia in patients with Bardet-Biedl or Alström syndrome have shown promising but inconsistent results in ADPKD (Fassett et al., 2010; Cadnapaphornchai et al., 2014; Brosnahan et al., 2017; Müller and Schermer, 2019). In addition, data should be collected to gain insight into ciliopathy-specific symptomatic treatment. For example, in a patient with NPHP3-related infantile $\mathrm{NPH}$ isosorbide dinitrate was used to treat hypertension by restoring nitric oxide generation (Strong et al., 2018). Nitric oxide is produced by vascular endothelial cells in response to ciliary sensation of shear stress and causes compensatory vasodilatation, a process that is impaired in ciliopathies. Targeting this cilia-specific cause of hypertension was more effective in this patient than classic anti-hypertensive medication (Strong et al., 2018).

Another consequence of the low prevalence and phenotypic heterogeneity in NPH is the need for personalized therapy. Developments in in vitro modeling, for example the use of URECs or the generation of kidney organoids from iPSCs, enable testing of medication directly in NPH patient-derived cells that harbor the causal mutation and the genetic background of the patient including modifier gene variants. This should facilitate translation of findings back to therapy in individual patients. Despite current limitations, improvement of NPHkidney organoids can aid drug development in NPH, as has been successfully illustrated in a kidney organoid model of cystinosis (Hollywood et al., 2020).

Patient-derived organoids can be combined with CRISPR/Cas9 technology to correct the NPH phenotype for various applications (Steichen et al., 2020). To overcome technical and ethical hurdles associated with in vivo therapeutic application of CRISPR/Cas9, genome editing can be applied ex vivo, for example to populate bioartificial kidneys with corrected patient-derived renal epithelial cells as a future alternative for donor kidneys if safety issues of cell-based therapies have been sufficiently resolved (McKee and Wingert, 2016; Mihajlovic et al., 2019a,b). Bioengineering strategies in kidney regeneration have been reviewed by Peired et al. (2020).

Besides ex vivo applications, developments in targeted delivery methods bring gene therapy in $\mathrm{NPH}$ patients one step closer (Liu et al., 2019). Whereas adeno-associated virus (AAV)-vector delivery of wildtype gene and CRISPR/Cas9 to retinal cells via subretinal injection has been demonstrated in Bbs4 and Cep290 mouse models, respectively, delivery of viral vector to the kidney via systemic injection is limited because AAV particles do not pass the glomerular filtration barrier (Simons et al., 2011; Ruan et al., 2017; Rubin et al., 2019). Targeted delivery and nephron segment-specific gene expression has been achieved via retrograde ureteral infusion of AAV9 vector in combination with segment-specific promoters in mice (Asico et al., 2018). This system of targeted delivery could be used for gene-based therapy in NPH. In addition, systemic administration of (artificial) extracellular vesicles or nanoparticles has been proposed for targeted delivery of siRNA or drugs to kidney cells (Barile and Vassalli, 2017; Oroojalian et al., 2020).

\section{CONCLUSION}

Many potential therapies for NPH have been explored in model systems, from targeted treatments aimed at restoring ciliary signaling, CDK inhibition, immune system suppression and ASO therapy to untargeted drug screens. Translation of results is limited by the lack of animal models that recapitulate the juvenile $\mathrm{NPH}$ phenotype and in the case of infantile $\mathrm{NPH}$, for which models exist, by the short therapeutic window. Employment of non-orthologous animal models and developments in organoid 
technology can potentially fill this gap and provide new opportunities for personalized treatments. Ultimately, safety and efficacy of potential NPH therapies will have to be tested in clinical trials. Given obstacles of genetic and phenotypic heterogeneity in renal ciliopathies and clinical trials in children, repurposing of medication used in other cystic or chronic kidney diseases could be the most viable approach. In addition to identification of effective drugs, successful treatment or prevention of NPH depends on the discovery of biomarkers that identify NPH patients in an early disease stage.

\section{AUTHOR CONTRIBUTIONS}

MS and $\mathrm{AB}$ conceived the idea and wrote the manuscript. $\mathrm{AB}$ and SS provided critical feedback. All authors contributed to the article and approved the submitted version.

\section{REFERENCES}

Acosta, J. C., Banito, A., Wuestefeld, T., Georgilis, A., Janich, P., Morton, J. P., et al. (2013). A complex secretory program orchestrated by the inflammasome controls paracrine senescence. Nat. Cell Biol. 15, 978-992.

Aguilar, A., Meunier, A., Strehl, L., Martinovic, J., Bonniere, M., Attie-Bitach, T., et al. (2012). Analysis of human samples reveals impaired SHH-dependent cerebellar development in Joubert syndrome/Meckel syndrome. Proc. Natl. Acad. Sci. U.S.A. 109, 16951-16956. doi: 10.1073/pnas.1201408109

Ahmadi, M., Amiri, S., Pecic, S., Machaj, F., Rosik, J., Łos, M. J., et al. (2020). Pleiotropic effects of statins: a focus on cancer. Biochim. Biophys. Acta Mol. Basis Dis. 2020:1866. doi: 10.1016/j.bbadis.2020.165968

Airik, R., Airik, M., Schueler, M., Bates, C. M., and Hildebrandt, F. (2019). Roscovitine blocks collecting duct cyst growth in Cep164-deficient kidneys. Kidney Int. 96, 320-326. doi: 10.1016/j.kint.2019.04.014

Airik, R., Schueler, M., Airik, M., Cho, J., Porath, J. D., Mukherjee, E., et al. (2016). A FANCD2/FANCI-associated nuclease 1-knockout model develops karyomegalic interstitial nephritis. J. Am. Soc. Nephrol. 27, 3552-3559. doi: 10.1681/ASN.2015101108

Airik, R., Slaats, G. G., Guo, Z., Weiss, A. C., Khan, N., Ghosh, A., et al. (2014). Renal-retinal ciliopathy gene Sdccag8 regulates DNA damage response signaling. J. Am. Soc. Nephrol. 25, 2573-2583. doi: 10.1681/ASN.2013050565

Akella, J. S., Carter, S. P., Nguyen, K., Tsiropoulou, S., Moran, A. L., Silva, M., et al. (2020). Ciliary rab28 and the BBSome negatively regulate extracellular vesicle shedding. eLife 9, 1-27. doi: 10.7554/eLife.50580

Alcaraz, W. A., Gold, D. A., Raponi, E., Gent, P. M., Concepcion, D., and Hamilton, B. A. (2006). Zfp423 controls proliferation and differentiation of neural precursors in cerebellar vermis formation. Proc. Natl. Acad. Sci. USA 103, 19424-19429. doi: 10.1073/pnas.0609184103

Anvarian, Z., Mykytyn, K., Mukhopadhyay, S., Pedersen, L. B., and Christensen, S. T. (2019). Cellular signalling by primary cilia in development, organ function and disease. Nat. Rev. Nephrol. 15, 199-219. doi: 10.1038/s41581-0190116-9

Arts, H. H., Doherty, D., Van Beersum, S. E. C., Parisi, M. A., Letteboer, S. J. F., Gorden, N. T., et al. (2007). Mutations in the gene encoding the basal body protein RPGRIP1L, a nephrocystin-4 interactor, cause Joubert syndrome. Nat. Genet. 39, 882-888. doi: 10.1038/ng2069

Ashe, A., Butterfield, N. C., Town, L., Courtney, A. D., Cooper, A. N., Ferguson, C., et al. (2012). Mutations in mouse Ift 144 model the craniofacial, limb and rib defects in skeletal ciliopathies. Hum. Mol. Genet. 21, 1808-1823. doi: 10.1093/ hmg/ddr613

Asico, L. D., Cuevas, S., Ma, X., Jose, P. A., Armando, I., and Konkalmatt, P. R. (2018). Nephron segment-specific gene expression using AAV vectors. Biochem. Biophys. Res. Commun. 497, 19-24. doi: 10.1016/j.bbrc.2018.01.169

\section{FUNDING}

The researchers received funding from the Dutch Kidney Foundation under grant agreement 19OK001 TACTICS to MS, the European Molecular Biology Organization (EMBO) ShortTerm Fellowship to MS and the Academy Ter Meulen grant of the Academy Medical Sciences Fund of the Royal Netherlands Academy of Arts and Sciences (KNAW) to MS, and from the Agence Nationale de la Recherche (ANR) to AB and SS, including the "Investissements d'Avenir" program (ANR-10-IAHU-01), and the "RHU-C'IL-LICO" as part of the second "Investissements d'Avenir" program (reference: ANR-17-RHUS-0002).

\section{ACKNOWLEDGMENTS}

The authors thank Amandine Viau for her constructive criticism of the manuscript.

Atala, A., Freeman, M. R., Mandell, J., and Beier, D. R. (1993). Juvenile cystic kidneys (jck): a new mouse mutation which causes polycystic kidneys. Kidney Int. 43, 1081-1085. doi: 10.1038/ki.1993.151

Attanasio, M., Uhlenhaut, N. H., Sousa, V. H., O’Toole, J. F., Otto, E., Anlag, K., et al. (2007). Loss of GLIS2 causes nephronophthisis in humans and mice by increased apoptosis and fibrosis. Nat. Genet. 39, 1018-1024.

Baar, M. P., Brandt, R. M., Putavet, D. A., Klein, J. D., Derks, K. W., Bourgeois, B. R., et al. (2017). Targeted apoptosis of senescent cells restores tissue homeostasis in response to chemotoxicity and aging. Cell 196, 132-147. doi: 10.1016/j.cell.2017. 02.031

Bakey, Z., Bihoreau, M. T., Piedagnel, R., Delestré, L., Arnould, C., De Villiers, A. D., et al. (2015). The SAM domain of ANKS6 has different interacting partners and mutations can induce different cystic phenotypes. Kidney Int. 88, 299-310. doi: 10.1038/ki.2015.122

Barile, L., and Vassalli, G. (2017). Exosomes: therapy delivery tools and biomarkers of diseases. Pharmacol. Ther. 174, 63-78. doi: 10.1016/j.pharmthera.2017.02. 020

Berbari, N. F., Kin, N. W., Sharma, N., Michaud, E. J., Kesterson, R. A., and Yoder, B. K. (2011). Mutations in Traf3ip1 reveal defects in ciliogenesis, embryonic development, and altered cell size regulation. Dev. Biol. 360, 66-76. doi: 10. 1016/j.ydbio.2011.09.001

Berbari, N. F., Sharma, N., Malarkey, E. B., Pieczynski, J. N., Boddu, R., Gaertig, J., et al. (2013). Microtubule modifications and stability are altered by cilia perturbation and in cystic kidney disease. Cytoskeleton 70, 24-31. doi: 10.1002/ cm. 21088

Bergmann, C. (2018). Genetics of autosomal recessive polycystic kidney disease and its differential diagnoses. Front. Pediatr. 5:221. doi: 10.3389/fped.2017. 00221

Bergmann, C., Fliegauf, M., Brüchle, N. O., Frank, V., Olbrich, H., Kirschner, J., et al. (2008). Loss of Nephrocystin-3 function can cause embryonic lethality, meckel-gruber-like syndrome, situs inversus, and renal-hepatic-pancreatic Dysplasia. Am. J. Hum. Genet. 82, 959-970. doi: 10.1016/j.ajhg.2008.02. 017

Billot, K., Coquil, C., Villiers, B., Josselin-Foll, B., Desban, N., Delehouzé, C., et al. (2018). Casein kinase $1 \varepsilon$ and $1 \alpha$ as novel players in polycystic kidney disease and mechanistic targets for (R)-roscovitine and (S)-CR8. Am. J. Physiol. Ren. Physiol. 315, F57-F73. doi: 10.1152/ajprenal.00489.2017

Bizet, A. A., Becker-Heck, A., Ryan, R., Weber, K., Filhol, E., Krug, P., et al. (2015). Mutations in TRAF3IP1/IFT54 reveal a new role for IFT proteins in microtubule stabilization. Nat. Commun. 6, 1-14. doi: 10.1038/ncomms 9666

Bockenhauer, D., van 't Hoff, W., Dattani, M., Lehnhardt, A., Subtirelu, M., Hildebrandt, F., et al. (2010). Secondary nephrogenic diabetes insipidus as a complication of inherited renal diseases. Bone 23, 1-7. doi: 10.1159/000320117 
Boehlke, C., Kotsis, F., Patel, V., Braeg, S., Bredt, S., Beyer, T., et al. (2010). Primary cilia regulate mTORC1 activity and cell size through Lkb1. Nat. Cell Biol. 12, 1115-1122. doi: 10.1038/ncb2117.Primary

Booij, T. H., Bange, H., Leonhard, W. N., Yan, K., Fokkelman, M., Kunnen, S. J., et al. (2017). High-throughput phenotypic screening of kinase inhibitors to identify drug targets for polycystic kidney disease. SLAS Discov. 22, 974-984. doi: $10.1177 / 2472555217716056$

Booij, T. H., Leonhard, W. N., Bange, H., Yan, K., Fokkelman, M., Plugge, A. J., et al. (2019). In vitro 3D phenotypic drug screen identifies celastrol as an effective in vivo inhibitor of polycystic kidney disease. J. Mol. Cell Biol. 12, 644-653. doi: $10.1093 / \mathrm{jmcb} / \mathrm{mjz} 029$

Boukhalfa, A., Miceli, C., Ávalos, Y., Morel, E., and Dupont, N. (2019). Interplay between primary cilia, ubiquitin-proteasome system and autophagy. Biochimie 166, 286-292. doi: 10.1016/j.biochi.2019.06.009

Bracken, C., Beauverger, P., Duclos, O., Russo, R. J., Rogers, K. A., Husson, H., et al. (2016). CaMKII as a pathological mediator of ER stress, oxidative stress, and mitochondrial dysfunction in a murine model of nephronophthisis. Am. J. Physiol. Ren. Physiol. 310, F1414-F1422. doi: 10.1152/ajprenal.00426.2015

Braun, D. A., and Hildebrandt, F. (2017). Ciliopathies. Cold Spring Harb. Perspect. Biol. 9:a028191.

Bredrup, C., Saunier, S., Oud, M. M., Fiskerstrand, T., Hoischen, A., Brackman, D., et al. (2011). Ciliopathies with skeletal anomalies and renal insufficiency due to mutations in the IFT-A gene WDR19. Am. J. Hum. Genet. 89, 634-643. doi: 10.1016/j.ajhg.2011.10.001

Breslow, D. K., Hoogendoorn, S., Kopp, A. R., Morgens, D. W., Vu, B. K., Kennedy, M. C., et al. (2018). A CRISPR-based screen for Hedgehog signaling provides insights into ciliary function and ciliopathies. Nat. Genet. 50, 460-471. doi: 10.1038/s41588-018-0054-7.A

Brosnahan, G. M., Abebe, K. Z., Rahbari-Oskoui, F. F., Patterson, C. G., Bae, K. T., Schrier, R. W., et al. (2017). Effect of statin therapy on the progression of autosomal dominant polycystic kidney disease. a secondary analysis of the HALT PKD Trials. Curr. Hypertens. Rev. 13, 109-120. doi: 10.2174/ 1573402113666170427142815

Brown, J. H., Bihoreau, M. T., Hoffmann, S., Kranzlin, B., Tychinskaya, I., Obermuller, N., et al. (2005). Missense mutation in sterile alpha motif of novel protein SamCystin is associated with polycystic kidney disease in (cy/+) rat. J. Am. Soc. Nephrol. 16, 3517-3526. doi: 10.1681/ASN.2005060601

Bukanov, N. O., Smith, L. A., Klinger, K. W., and Ledbetter, S. R. (2006). Long-lasting arrest of murine polycystic kidney disease with CDK inhibitor roscovitine. Nature 444, 949-952. doi: 10.1038/nature05348

Burckle, C., Gaude, H. M., Vesque, C., Silbermann, F., Salomon, R., Jeanpierre, C., et al. (2011). Control of the Wnt pathways by nephrocystin-4 is required for morphogenesis of the zebrafish pronephros. Hum. Mol. Genet. 20, 2611-2627. doi: $10.1093 / \mathrm{hmg} / \mathrm{ddr} 164$

Cadnapaphornchai, M. A., George, D. M., McFann, K., Wang, W., Gitomer, B., Strain, J. D., et al. (2014). Effect of pravastatin on total kidney volume, left ventricular mass index, and microalbuminuria in pediatric autosomal dominant polycystic kidney disease. Clin. J. Am. Soc. Nephrol. 9, 889-896. doi: 10.2215/CJN.08350813

Cao, Q., Harris, D. C. H., and Wang, Y. (2015). Macrophages in kidney injury, inflammation, and fibrosis. Physiology 30, 183-194. doi: 10.1152/physiol.00046. 2014

Cao, Y., Semanchik, N., Lee, S. H., Somlo, S., Barbano, P. E., Coifman, R., et al. (2009). Chemical modifier screen identifies HDAC inhibitors as suppressors of PKD models. Proc. Natl. Acad. Sci. U.S.A. 106, 21819-21824. doi: 10.1073/pnas. 0911987106

Caroli, A., Antiga, L., Cafaro, M., Fasolini, G., and Remuzzi, A. (2010). Reducing polycystic liver volume in ADPKD: effects of somatostatin analogue octreotide. Clin. J. Am. Soc. Nephrol. 5, 783-789. doi: 10.2215/CJN.05380709

Casoni, F., Croci, L., Bosone, C., D’Ambrosio, R., Badaloni, A., Gaudesi, D., et al. (2017). Zfp423/ZNF423 regulates cell cycle progression, the mode of cell division and the DNA-damage response in purkinje neuron progenitors. Dev. 144, 3686-3697. doi: 10.1242/dev.155077

Chaki, M., Airik, R., Ghosh, A. K., Giles, R. H., Chen, R., Slaats, G. G., et al. (2012). Exome capture reveals ZNF423 and CEP164 mutations, linking renal ciliopathies to DNA damage response signaling. Cell 150, 533-548. doi: 10.1016/ j.cell.2012.06.028.Exome
Chang, B., Khanna, H., Hawes, N., Jimeno, D., He, S., Lillo, C., et al. (2006). Inframe deletion in a novel centrosomal/ciliary protein CEP290/ NPHP6 perturbs its interaction with RPGR and results in early- onset retinal degeneration in the rd16 mouse. Hum. Mol. Genet. 15, 1847-1857.

Chatterjee, S., Shi, W. Y., Wilson, P., and Mazumdar, A. (1996). Role of lactosylceramide and MAP kinase in the proliferation of proximal tubular cells in human polycystic kidney disease. J. Lipid Res. 37, 1334-1344.

Chebib, F. T., Perrone, R. D., Chapman, A. B., Dahl, N. K., Harris, P. C., Mrug, M., et al. (2018). A practical guide for treatment of rapidly progressive ADPKD with tolvaptan. J. Am. Soc. Nephrol. 29, 2458-2470. doi: 10.1681/ASN.2018060590

Chebib, F. T., and Torres, V. E. (2016). Autosomal dominant polycystic kidney disease: core curriculum 2016. Am. J. Kidney Dis. 67, 792-810. doi: 10.1053/j. ajkd.2015.07.037

Chen, N. X., Moe, S. M., Eggleston-Gulyas, T., Chen, X., Hoffmeyer, W. D., Bacallao, R. L., et al. (2011). Calcimimetics inhibit renal pathology in rodent nephronophthisis. Kidney Int. 80, 612-619. doi: 10.1038/ki.2011.139

Cheng, L. E., Zhang, J., and Reed, R. R. (2007). The transcription factor Zfp $423 / O A Z$ is required for cerebellar development and CNS midline patterning. Dev. Biol. 307, 43-52. doi: 10.1016/j.ydbio.2007.04.005

Choi, H. J. C., Lin, J. R., Vannier, J. B., Slaats, G. G., Kile, A. C., Paulsen, R. D., et al. (2013). NEK8 links the ATR-regulated replication stress response and S phase CDK activity to renal ciliopathies. Mol. Cell 51, 423-439. doi: 10.1016/j.molcel. 2013.08.006

Choi, Y. J., Halbritter, J., Braun, D. A., Schueler, M., Schapiro, D., Rim, J. H., et al. (2019). Mutations of ADAMTS9 cause nephronophthisis-related ciliopathy. Am. J. Hum. Genet. 104, 45-54. doi: 10.1016/j.ajhg.2018.11.003

Cideciyan, A. V., Jacobson, S. G., Drack, A. V., Ho, A. C., Charng, J., Garafalo, A. V., et al. (2019). Effect of an intravitreal antisense oligonucleotide on vision in Leber congenital amaurosis due to a photoreceptor cilium defect. Nat. Med. 25, 225-228. doi: 10.1038/s41591-018-0295-0

Collin, R. W., Den Hollander, A. I., Der Velde-Visser, S. D. V., Bennicelli, J., Bennett, J., and Cremers, F. P. (2012). Antisense oligonucleotide (AON)based therapy for leber congenital amaurosis caused by a frequent mutation in CEP290. Mol. Ther. Nucleic Acids 1, e14. doi: 10.1038/mtna.2012.3

Cong, E. H., Bizet, A. A., Boyer, O., Woerner, S., Gribouval, O., Filhol, E., et al. (2014). A Homozygous missense mutation in the ciliary gene TTC21B causes familial FSGS. J. Am. Soc. Nephrol. 25, 1-9. doi: 10.1681/ASN.2013101126

Cook, S. A., Collin, G. B., Bronson, R. T., Naggert, J. K., Liu, D. P., Akeson, E. C., et al. (2009). A mouse model for meckel syndrome type 3. J. Am. Soc. Nephrol. 20, 753-764. doi: 10.1681/ASN.2008040412

Cowley, B. D., Gudapaty, S., Kraybill, A. L., Barash, B. D., Harding, M. A., Calvet, J. P., et al. (1993). Autosomal-dominant polycystic kidney disease in the rat. Kidney Int. 43, 522-534. doi: 10.1038/ki.1993.79

Cruz, N. M., Song, X., Czerniecki, S. M., Gulieva, R. E., Churchill, A. J., Kim, Y. K., et al. (2017). Organoid cystogenesis reveals a critical role of microenvironment in human polycystic kidney disease. Nat. Mater. 16, 1112-1119. doi: 10.1038/ NMAT4994

Davis, E. E., Zhang, Q., Liu, Q., Diplas, B. H., Davey, L. M., Hartley, J., et al. (2011). TTC21B contributes both causal and modifying alleles across the ciliopathy spectrum. Nat. Genet. 43, 189-196. doi: 10.1038/ng.756

Delous, M., Baala, L., Salomon, R., Laclef, C., Vierkotten, J., Tory, K., et al. (2007). The ciliary gene RPGRIP1L is mutated in cerebello-oculo-renal syndrome (Joubert syndrome type B) and Meckel syndrome. Nat. Genet. 39, 875-881.

Delous, M., Hellman, N. E., Gaudé, H. M., Silbermann, F., Le Bivic, A., Salomon, R., et al. (2009). Nephrocystin-1 and nephrocystin-4 are required for epithelial morphogenesis and associate with PALS1/PATJ and Par6. Hum. Mol. Genet. 18, 4711-4723. doi: 10.1093/hmg/ddp434

Deshmukh, G. D., Radin, N. S., Gattone, V. H., and Shayman, J. A. (1994). Abnormalities of glycosphingolipid, sulfatide, and ceramide in the polycystic (cpk/cpk) mouse. J. Lipid Res. 35, 1611-1618.

Devlin, L. A., and Sayer, J. A. (2019). Renal ciliopathies. Curr. Opin. Genet. Dev. 56, 49-60. doi: 10.1016/j.gde.2019.07.005

Devuyst, O., Olinger, E., Weber, S., Eckardt, K. U., Kmoch, S., Rampoldi, L., et al. (2019). Autosomal dominant tubulointerstitial kidney disease. Nat. Rev. Dis. Prim. 5:60. doi: 10.1038/s41572-019-0109-9

Ding, B., Sun, G., Liu, S., Peng, E., Wan, M., Chen, L., et al. (2020). Threedimensional renal organoids from whole kidney cells: generation, optimization, 
and potential application in nephrotoxicology in vitro. Cell Transplant. 29, 1-10. doi: $10.1177 / 0963689719897066$

Distler, J. H. W., Györfi, A. H., Ramanujam, M., Whitfield, M. L., Königshoff, M., and Lafyatis, R. (2019). Shared and distinct mechanisms of fibrosis. Nat. Rev. Rheumatol. 15, 705-730. doi: 10.1038/s41584-019-0322-7

Docherty, M. H., O'Sullivan, E. D., Bonventre, J. V., and Ferenbach, D. A. (2019). Cellular senescence in the kidney. J. Am. Soc. Nephrol. 30, 726-736. doi: 10. 1681/ASN.2018121251

Donaldson, J. C., Dise, R. S., Ritchie, M. D., and Hanks, S. K. (2002). Nephrocystinconserved domains involved in targeting to epithelial cell-cell junctions, interaction with filamins, and establishing cell polarity. J. Biol. Chem. 277, 29028-29035. doi: 10.1074/jbc.M111697200

Drivas, T. G., Wojno, A. P., Tucker, B. A., Stone, E. M., and Bennett, J. (2015), Basal exon skipping and genetic pleiotropy: a predictive model of disease pathogenesis. Sci. Transl. Med. 7:291ra97. doi: 10.1126/scitranslmed.aaa5370

Dubail, J., Aramaki-Hattori, N., Bader, H. L., Nelson, C. M., Katebi, N., Matuska, B., et al. (2014). A new Adamts 9 conditional mouse allele identifies its nonredundant role in interdigital web regression. Genesis 52, 702-712. doi: 10.1002/ dvg. 22784

Ecder, T. (2016). Statins in the treatment of autosomal dominant polycystic kidney disease. Nephrol. Dial. Transplant. 31, 1194-1196. doi: 10.1093/ndt/gfv449

Elberg, G., Elberg, D., Lewis, T. V., Guruswamy, S., Chen, L., Logan, C. J., et al. (2007). EP2 receptor mediates PGE2-induced cystogenesis of human renal epithelial cells. Am. J. Physiol. Ren. Physiol. 293, 1622-1632. doi: 10.1152/ ajprenal.00036.2007

El-Brolosy, M. A., and Stainier, D. Y. R. (2017). Genetic compensation: a phenomenon in search of mechanisms. PLoS Genet. 13:e1006780. doi: 10.1371/ journal.pgen.1006780

Elmonem, M., Berlingerio, S., van den Heuvel, L., de Witte, P., Lowe, M., and Levtchenko, E. (2018). Genetic renal diseases: the emerging role of zebrafish models. Cells 7:130. doi: 10.3390/cells7090130

Estrada-Cuzcano, A., Koenekoop, R. K., Coppieters, F., Kohl, S., Lopez, I., Collin, R. W. J., et al. (2011). IQCB1 mutations in patients with leber congenital amaurosis. Investig. Ophthalmol. Vis. Sci. 52, 834-839. doi: 10.1167/iovs.105221

Failler, M., Gee, H. Y., Krug, P., Joo, K., Halbritter, J., Belkacem, L., et al. (2014). Mutations of CEP83 cause infantile nephronophthisis and intellectual disability. Am. J. Hum. Genet. 94, 905-914. doi: 10.1016/j.ajhg.2014.05.002

Fassett, R. G., Coombes, J. S., Packham, D., Fairley, K. F., and Kincaid-Smith, P. (2010). Effect of pravastatin on kidney function and urinary protein excretion in autosomal dominant polycystic kidney disease. Scand. J. Urol. Nephrol. 44, 56-61. doi: 10.3109/00365590903359908

Fay, A. J., Knox, R., Neil, E. E., and Strober, J. (2020). Targeted treatments for inherited neuromuscular diseases of childhood. Semin. Neurol. 40, 335-341.

Fischer, E., and Pontoglio, M. (2009). Planar cell polarity and cilia. Semin. Cell Dev. Biol. 20, 998-1005. doi: 10.1016/j.semcdb.2009.09.016

Fitzgibbon, W. R., Dang, Y., Bunni, M. A., Baicu, C. F., Zile, M. R., Mullick, A. E., et al. (2018). Attenuation of accelerated renal cystogenesis in pkd1 mice by renin-angiotensin system blockade. Am. J. Physiol. Ren. Physiol. 314, F210-F218. doi: 10.1152/ajprenal.00389.2017

Forbes, T. A., Howden, S. E., Lawlor, K., Phipson, B., Maksimovic, J., Hale, L., et al. (2018). Patient-iPSC-derived kidney organoids show functional validation of a ciliopathic renal phenotype and reveal underlying pathogenetic mechanisms. Am. J. Hum. Genet. 102, 816-831. doi: 10.1016/j.ajhg.2018.03.014

Forsythe, E., Kenny, J., Bacchelli, C., and Beales, P. L. (2018). Managing bardet biedl syndrome - now and in the future. Front. Pediatr. 6:23. doi: 10.3389/fped. 2018.00023

Frank, V., Habbig, S., Bartram, M. P., Eisenberger, T., Veenstra-Knol, H. E., Decker, C., et al. (2013). Mutations in NEK8 link multiple organ dysplasia with altered Hippo signalling and increased c-MYC expression. Hum. Mol. Genet. 22, 2177-2185. doi: 10.1093/hmg/ddt070

Gagnadoux, M. F., Bacri, J. L., Broyer, M., and Habib, R. (1989). Infantile chronic tubulo-interstitial nephritis with cortical microcysts: variant of nephronophthisis or new disease entity? Pediatr. Nephrol. 3, 50-55. doi: 10. 1007/BF00859626

Gattone, V. H. II, Sinders, R. M., Hornberger, T. A., and Robling, A. G. (2009). Late progression of renal pathology and cyst enlargement is reduced by rapamycin in a mouse model of nephronophthisis. Kidney Int. 76, 178-182. doi: 10.1038/ ki.2009.147

Gattone, V. H. II, Wang, X., Harris, P. C., and Torres, V. E. (2003). Inhibition of renal cystic disease development and progression by a vasopressin V2 receptor antagonist. Nat. Med. 9, 1323-1326. doi: 10.1038/nm935

Gattone, V. H., Cowley, B. D., Barash, B. D., Nagao, S., Takahashi, H., Yamaguchi, T., et al. (1995). Methylprednisolone retards the progression of inherited polycystic kidney disease in rodents. Am. J. Kidney Dis. 25, 302-313. doi: 10. 1016/0272-6386(95)90013-6

Gattone, V. H., Tourkow, B. A., Trambaugh, C. M., Yu, A. C., Whelan, S., Phillips, C. L., et al. (2004). Development of multiorgan pathology in the wpk rat model of polycystic kidney disease. Anat. Rec. Part A Discov. Mol. Cell. Evol. Biol. 277, 384-395. doi: 10.1002/ar.a.20022

Gehrig, J., Pandey, G., and Westhoff, J. H. (2018). Zebrafish as a model for drug screening in genetic kidney diseases. Front. Pediatr. 6:183. doi: 10.3389/fped. 2018.00183

Gerard, X., Perrault, I., Hanein, S., Silva, E., Bigot, K., Defoort-Delhemmes, S., et al. (2012). AON-mediated exon skipping restores ciliation in fibroblasts harboring the common leber congenital amaurosis CEP290 mutation. Mol. Ther. Nucleic Acids 1:e29. doi: 10.1038/mtna.2012.21

Ghosh, A. K., Hurd, T., and Hildebrandt, F. (2012). 3D spheroid defects in NPHP knockdown cells are rescued by the somatostatin receptor agonist octreotide. Am. J. Physiol. Ren. Physiol. 303, 1225-1229. doi: 10.1152/ajprenal.00135.2012

Gile, R. D., Cowley, B. D., Gattone, V. H., O’Donnell, M. P., Swan, S. K., and Grantham, J. J. (1995). Effect of lovastatin on the development of polycystic kidney disease in the han:SPRD rat. Am. J. Kidney Dis. 26, 501-507. doi: 10. 1016/0272-6386(95)90497-2

Gorivodsky, M., Mukhopadhyay, M., Wilsch-Braeuninger, M., Phillips, M., Teufel, A., Kim, C., et al. (2009). Intraflagellar transport protein 172 is essential for primary cilia formation and plays a vital role in patterning the mammalian brain. Dev. Biol. 325, 24-32. doi: 10.1016/j.ydbio.2008.09.019

Goru, S. K. S. (2018). Novel reno-protective mechanism of aspirin involves H2AK119 monoubiquitination and Set7 in preventing type 1 diabetic nephropathy. Pharmacol. Rep. 70, 497-502.

Grampa, V., Delous, M., Zaidan, M., Odye, G., Thomas, S., Elkhartoufi, N., et al. (2016). Novel NEK8 mutations cause severe syndromic renal cystic dysplasia through YAP Dysregulation. PLoS Genet. 12:e1005894. doi: 10.1371/journal. pgen.1005894

Günther, T., Tulipano, G., Dournaud, P., Bousquet, C., Csaba, Z., Kreienkamp, H. J., et al. (2018). International union of basic and clinical pharmacology. CV. somatostatin receptors: structure, function, ligands, and new nomenclature. Pharmacol. Rev. 70, 763-835. doi: 10.1124/pr.117.015388

Habbig, S., Bartram, M. P., Muller, R. U., Schwarz, R., Andriopoulos, N., Chen, S., et al. (2011). NPHP4, a cilia-associated protein, negatively regulates the Hippo pathway. J. Cell Biol. 193, 633-642. doi: 10.1083/jcb.201009069

Habbig, S., Bartram, M. P., Sägmüller, J. G., Griessmann, A., Franke, M., Müller, R. U., et al. (2012). The ciliopathy disease protein NPHP9 promotes nuclear delivery and activation of the oncogenic transcriptional regulator TAZ. Hum. Mol. Genet. 21, 5528-5538. doi: 10.1093/hmg/dds408

Halbritter, J., Bizet, A. A., Schmidts, M., Porath, J. D., Braun, D. A., Gee, H. Y., et al. (2013a). Defects in the IFT-B component IFT172 cause jeune and mainzersaldino syndromes in humans. Am. J. Hum. Genet. 93, 915-925. doi: 10.1016/j. ajhg.2013.09.012

Halbritter, J., Porath, J. D., Diaz, K. A., Braun, D. A., Kohl, S., Chaki, M., et al. (2013b). Identification of 99 novel mutations in a worldwide cohort of 1,056 patients with a nephronophthisis-related ciliopathy. Hum. Genet. 132, 865-884. doi: 10.1007/s00439-013-1297-0

Hamiwka, L. A., Midgley, J. P., Wade, A. W., Martz, K. L., and Grisaru, S. (2008). Outcomes of kidney transplantation in children with nephronophthisis: an analysis of the North American Pediatric Renal Trials and Collaborative Studies (NAPRTCS) Registry. Pediatr. Transplant. 12, 878-882. doi: 10.1111/j.13993046.2008.00942.x

Han, S. H., Malaga-DIeguez, L., Chinga, F., Kang, H. M., Tao, J., Reidy, K., et al. (2016). Deletion of Lkb1 in renal tubular epithelial cells leads to CKD by altering metabolism. J. Am. Soc. Nephrol. 27, 439-453. doi: 10.1681/ASN.2014121181

Hansen, J. N., Kaiser, F., Klausen, C., Stüven, B., Chong, R., Bönigk, W., et al. (2020). Nanobody-directed targeting of optogenetic tools reveals differential 
regulation of cilia length. bioRxiv [Preprint]. doi: 10.1101/2020.02.04.93 3440

Harris, P. C., and Torres, V. E. (2002). Autosomal dominant polycystic kidney disease. Gene Rev. 2, 2645-2688. doi: 10.1016/B978-0-12-381462-3.00080-X

Helou, J., Otto, E. A., Attanasio, M., Allen, S. J., Parisi, M. A., Glass, I., et al. (2007). Mutation analysis of NPHP6/CEP290 in patients with Joubert syndrome ana Senior-Løken syndrome. J. Med. Genet. 44, 657-663. doi: 10.1136/jmg.2007. 052027

Hildebrandt, F., Attanasio, M., and Otto, E. (2009). Nephronophthisis: disease mechanisms of a ciliopathy. J. Am. Soc. Nephrol. 20, 23-35. doi: 10.1681/ASN. 2008050456

Hildebrandt, F., Otto, E., Rensing, C., Nothwang, H. G., Vollmer, M., Adolphs, J., et al. (1997). A novel gene encoding an SH3 domain protein is mutated in nephronophthisis type 1. Nat. Genet. 17, 149-153. doi: 10.1038/ng1097149

Hoff, S., Halbritter, J., Epting, D., Frank, V., Nguyen, T.-M. T., van Reeuwijk, J., et al. (2013). ANKS6 is a central component of a nephronophthisis module linking NEK8 to INVS and NPHP3. Nat. Genet. 45, 951-958.

Hogan, M. C., Masyuk, T. V., Page, L. J., Kubly, V. J., Bergstralh, E. J., Li, X., et al. (2010). Randomized clinical trial of long-acting somatostatin for autosomal dominant polycystic kidney and liver disease. J. Am. Soc. Nephrol. 21, 1052-1061. doi: 10.1681/ASN.2009121291

Hollywood, J. A., Przepiorski, A., D’Souza, R. F., Sreebhavan, S., Wolvetang, E. J., Harrison, P. T., et al. (2020). Use of human induced pluripotent stem cells and kidney organoids to develop a Cysteamine/mTOR inhibition combination therapy for cystinosis. J. Am. Soc. Nephrol. 2020:712. doi: 10.1681/ asn. 2019070712

Hong, C. J., and Hamilton, B. A. (2016). Zfp423 regulates sonic hedgehog signaling via primary cilium function. PLoS Genet. 12:e1006357. doi: 10.1371/journal. pgen. 1006357

Hurd, T. W., Otto, E. A., Mishima, E., Gee, H. Y., Inoue, H., Inazu, M., et al. (2013). Mutation of the $\mathrm{Mg} 2+$ transporter SLC41A1 results in a nephronophthisis-like phenotype. J. Am. Soc. Nephrol. 24, 967-977. doi: 10.1681/ASN.2012101034

Husson, H., Moreno, S., Smith, L. A., Smith, M. M., Russo, R. J., Pitstick, R., et al. (2016). Reduction of ciliary length through pharmacologic or genetic inhibition of CDK5 attenuates polycystic kidney disease in a model of nephronophthisis. Hum. Mol. Genet. 25, 2245-2255. doi: 10.1093/hmg/ddw093

Hynes, A. M., Giles, R. H., Srivastava, S., Eley, L., Whitehead, J., Danilenko, M., et al. (2014). Murine Joubert syndrome reveals Hedgehog signaling defects as a potential therapeutic target for nephronophthisis. Proc. Natl. Acad. Sci. U.S.A. 111, 9893-9898. doi: 10.1073/pnas.1322373111

Ibraghimov-Beskrovnaya, O. (2007). Targeting dysregulated cell cycle and apoptosis for polycystic kidney disease therapy. Cell Cycle 6, 776-779. doi: 10.4161/cc.6.7.4047

Ibrahim, N. H. M., Gregoire, M., Devassy, J. G., Wu, Y., Yoshihara, D., Yamaguchi, T., et al. (2015). Cyclooxygenase product inhibition with acetylsalicylic acid slows disease progression in the Han:SPRD-Cy rat model of polycystic kidney disease. Prostagland. Other Lipid Mediat. 116-117, 19-25. doi: 10.1016/j. prostaglandins.2014.10.005

Ibrahim, N. H. M., Jia, Y., Devassy, J. G., Yamaguchi, T., and Aukema, H. M. (2014). Renal cyclooxygenase and lipoxygenase products are altered in polycystic kidneys and by dietary soy protein and fish oil treatment in the Han: SPRD-Cy rat. Mol. Nutr. Food Res. 58, 768-781. doi: 10.1002/mnfr.201300332

Jain, M., Kaiser, R. W. J., Bohl, K., Hoehne, M., Göbel, H., Bartram, M. P., et al. (2019). Inactivation of apoptosis antagonizing transcription factor in tubular epithelial cells induces accumulation of DNA damage and Nephronophthisis. Kidney Int. 95, 846-858. doi: 10.1016/j.kint.2018.10.034

Jiang, J. Y., Falcone, J. L., Curci, S., and Hofer, A. M. (2019). Direct visualization of cAMP signaling in primary cilia reveals up-regulation of ciliary GPCR activity following Hedgehog activation. Proc. Natl. Acad. Sci. U.S.A. 116, 12066-12071. doi: 10.1073/pnas.1819730116

Jiang, S. T., Chiou, Y. Y., Wang, E., Lin, H. K., Lee, S. P., Lu, H. Y., et al. (2008). Targeted disruption of Nphp1 causes male infertility due to defects in the later steps of sperm morphogenesis in mice. Hum. Mol. Genet. 17, 3368-3379. doi: $10.1093 / \mathrm{hmg} / \mathrm{ddn} 231$

Jin, D., Ni, T. T., Sun, J., Wan, H., Amack, J. D., Yu, G., et al. (2014). Prostaglandin signalling regulates ciliogenesis by modulating intraflagellar transport. Nat. Cell Biol. 16, 841-851. doi: 10.1038/ncb3029
Jin, H., Zhang, Y., Ding, Q., Wang, S. S., Rastogi, P., Dai, D. F., et al. (2019). Epithelial innate immunity mediates tubular cell senescence after kidney injury. JCI Insight 4, 1-14. doi: 10.1172/jci.insight.125490

Jin, H., Zhang, Y., Liu, D., Wang, S. S., Ding, Q., Rastogi, P., et al. (2020). Innate immune signaling contributes to tubular cell senescence in the glis2 knockout mouse model of Nephronophthisis. Am. J. Pathol. 190, 176-189. doi: 10.1016/j. ajpath.2019.09.013

Ke, B., Chen, Y., Tu, W., Ye, T., Fang, X., and Yang, L. (2018). Inhibition of HDAC6 activity in kidney diseases: a new perspective. Mol. Med. 24, 1-6. doi: 10.1186/s10020-018-0027-4

Kern, C. B., Wessels, A., McGarity, J., Dixon, L. J., Alston, E., Argraves, W. S., et al. (2010). Reduced versican cleavage due to Adamts9 haploinsufficiency is associated with cardiac and aortic anomalies. Matrix Biol. 29, 304-316. doi: 10.1016/j.matbio.2010.01.005

Kim, J., Lee, J. E., Heynen-Genel, S., Suyama, E., Ono, K., Lee, K., et al. (2010). Functional genomic screen for modulators of ciliogenesis and cilium length. Nature 464, 1048-1051. doi: 10.1038/nature08895

Kim, Y. J., and Kim, J. (2019). Therapeutic perspectives for structural and functional abnormalities of cilia. Cell. Mol. Life Sci. 76, 3695-3709. doi: 10.1007/ s00018-019-03158-6

Kim, Y. J., Kwon, H. J., Kim, J., Kim, Y. J., Kim, S., Jung, Y., et al. (2018). Eupatilin rescues ciliary transition zone defects to ameliorate ciliopathy-related phenotypes. J. Clin. Invest. 128, 3642-3648.

Kim, Y.-S., Kang, H. S., Herbert, R., Beak, J. Y., Collins, J. B., Grissom, S. F., et al. (2008). Krüppel-like zinc finger protein Glis2 is essential for the maintenance of normal renal functions. Mol. Cell. Biol. 28, 2358-2367. doi: 10.1128/mcb.0172207

Ko, J. Y., and Park, J. H. (2013). Mouse models of polycystic kidney disease induced by defects of ciliary proteins. BMB Rep. 46, 73-79. doi: 10.5483/BMBRep.2013. 46.2.022

Kong, J. H., Siebold, C., and Rohatgi, R. (2019). Biochemical mechanisms of vertebrate hedgehog signaling. Development 146:dev.166892. doi: 10.1242/dev. 166892

König, J., Kranz, B., König, S., Schlingmann, K. P., Titieni, A., Tönshoff, B., et al. (2017). Phenotypic spectrum of children with nephronophthisis and related Ciliopathies. Clin. J. Am. Soc. Nephrol. 12, 1974-1983. doi: 10.2215/CJN. 01280217

Kopinke, D., Norris, A. M., and Mukhopadhyay, S. (2020). Developmental and regenerative paradigms of cilia regulated hedgehog signaling. Semin. Cell Dev. Biol. 110, 89-103. doi: 10.1016/j.semcdb.2020.05.029

Krishnan, R., Eley, L., and Sayer, J. A. (2008). Urinary concentration defects and mechanisms underlying nephronophthisis. Kidney Blood Press. Res. 31, 152-162. doi: 10.1159/000129648

Kuraoka, S., Tanigawa, S., Taguchi, A., Hotta, A., Nakazato, H., Osafune, K., et al. (2020). PKD1-dependent renal cystogenesis in human induced pluripotent stem cell-derived ureteric bud/collecting duct organoids. J Am Soc Nephrol 31, 2355-2371. doi: 10.1681/ASN.2020030378

Lai, Y., and Jiang, Y. (2020). Reciprocal regulation between primary cilia and mtorc1. Genes 11, 1-15. doi: 10.3390/genes11060711

Lakhia, R., Mishra, A., and Patel, V. (2019). Manipulation of Renal Gene Expression using Oligonucleotides, 1st Edn, Amsterdam: Elsevier Inc, doi: 10.1016/bs.mcb. 2019.05.006

Lancaster, M. A., Louie, C. M., Silhavy, J. L., Sintasath, L., DeCambre, M., Nigam, S. K., et al. (2009). Impaired Wnt- $\beta$-catenin signaling disrupts adult renal homeostasis and leads to cystic kidney ciliopathy. Nat. Med. 15, 1046-1054. doi: $10.1038 / \mathrm{nm} .2010$. Impaired

Lannoy, M., Valluru, M. K., Chang, L., Abdela-Ali, F., Peters, D. J. M., Streets, A. J., et al. (2020). The positive effect of selective prostaglandin E2 receptor EP2 and EP4 blockade on cystogenesis in vitro is counteracted by increased kidney inflammation in vivo. Kidney Int. 98, 404-419. doi: 10.1016/j.kint.2020.02.012

Lemos, D. R., McMurdo, M., Karaca, G., Wilflingseder, J., Leaf, I. A., Gupta, N., et al. (2018). Interleukin-1b activates a MYC-dependent metabolic switch in kidney stromal cells necessary for progressive tubulointerstitial fibrosis. J. Am. Soc. Nephrol. 29, 1690-1705. doi: 10.1681/ASN.2017121283

Li, B., Rauhauser, A. A., Dai, J., Sakthivel, R., Igarashi, P., Jetten, A. M., et al. (2011). Increased hedgehog signaling in postnatal kidney results in aberrant activation of nephron developmental programs. Hum. Mol. Genet. 20, 4155-4166. doi: $10.1093 / \mathrm{hmg} / \mathrm{ddr} 339$ 
Li, J. H., Chou, C. L., Li, B., Gavrilova, O., Eisner, C., Schnermann, J., et al. (2009). A selective EP4 PGE2 receptor agonist alleviates disease in a new mouse model of X-linked nephrogenic diabetes insipidus. J. Clin. Invest. 119, 3115-3126. doi: $10.1172 /$ JCI39680

Li, Y., Xia, W., Zhao, F., Wen, Z., Zhang, A., Huang, S., et al. (2018). Prostaglandins in the pathogenesis of kidney diseases. Oncotarget 9, 26586-26602. doi: 10. 18632/oncotarget.25005

Liu, C. P., Hu, Y., Lin, J. C., Fu, H. L., Lim, L. Y., and Yuan, Z. X. (2019). Targeting strategies for drug delivery to the kidney: from renal glomeruli to tubules. Med. Res. Rev. 39, 561-578. doi: 10.1002/med.21532

Liu, S., Lu, W., Obara, T., Kuida, S., Lehoczky, J., Dewar, K., et al. (2002). A defect in novel Nek-family kinase causes cystic kidney disease in the mouse and in zebrafish. Development 129, 5839-5846. doi: 10.1242/dev.00173

Loktev, A. V., Zhang, Q., Beck, J. S., Searby, C. C., Scheetz, T. E., Bazan, J. F., et al. (2008). A BBSome subunit links ciliogenesis, microtubule stability, and Acetylation. Dev. Cell 15, 854-865. doi: 10.1016/j.devcel.2008.11.001

Low, J. H., Li, P., Chew, E. G. Y., Zhou, B., Suzuki, K., Zhang, T., et al. (2019). Generation of human PSC-derived kidney organoids with patterned nephron segments and a de novo vascular network. Cell Stem Cell 25, 373-387.e9. doi: 10.1016/j.stem.2019.06.009

Lowe, L. A., Supp, D. M., Sampath, K., Yokoyama, T., Wright, C. V. E., Potter, S. S., et al. (1996). Conserved left-right asymmetry of nodal expression and alterations in murine situs inversus. Nature 381, 158-161. doi: 10.1038/ $381158 \mathrm{a} 0$

Lu, D., Rauhauser, A., Li, B., Ren, C., McEnery, K., Zhu, J., et al. (2016). Loss of Glis2/NPHP7 causes kidney epithelial cell senescence and suppresses cyst growth in the Kif3a mouse model of cystic kidney disease. Kidney Int. 89, 1307-1323. doi: 10.1016/j.kint.2016.03.006

Luo, F., and Tao, Y. H. (2018). Nephronophthisis: A review of genotype-phenotype correlation. Nephrology 23, 904-911. doi: 10.1111/nep.13393

Ma, M., Legué, E., Tian, X., Somlo, S., and Liem, K. F. (2019). Cell-autonomous hedgehog signaling is not required for cyst formation in autosomal dominant polycystic kidney disease. J. Am. Soc. Nephrol. 30, 2103-2111. doi: 10.1681/ASN. 2018121274

Macia, M. S., Halbritter, J., Delous, M., Bredrup, C., Gutter, A., Filhol, E., et al. (2017). Mutations in MAPKBP1 cause juvenile or late-onset cilia-independent Nephronophthisis. Am. J. Hum. Genet. 100, 323-333. doi: 10.1016/j.ajhg.2016. 12.011

Maerz, L. D., Burkhalter, M. D., Schilpp, C., Wittekindt, O. H., Frick, M., and Philipp, M. (2019). Pharmacological cholesterol depletion disturbs ciliogenesis and ciliary function in developing zebrafish. Commun. Biol. 2, 1-13. doi: 10 . 1038/s42003-018-0272-7

Mahuzier, A., Gaudé, H. M., Grampa, V., Anselme, I., Silbermann, F., LerouxBerger, M., et al. (2012). Dishevelled stabilization by the ciliopathy protein rpgrip1l is essential for planar cell polarity. J. Cell Biol. 198, 927-940. doi: $10.1083 /$ jcb.201111009

Malik, S. A., Modarage, K., and Goggolidou, P. (2020). The role of WNT signalling in chronic kidney disease (CKD). Genes 11, 1-19. doi: 10.3390/genes11050496

Mangoo-Karim, R., Uchic, M. E., Grant, M., Shumate, W. A., Calvet, J. P., Park, C. H., et al. (1989). Renal epithelial fluid secretion and cyst growth: the role of cyclic AMP. FASEB J. 3, 2629-2632. doi: 10.1096/fasebj.3.14.2480260

Manning, D. K., Sergeev, M., Van Heesbeen, R. G., Wong, M. D., Oh, J. H., Liu, Y., et al. (2013). Loss of the ciliary kinase Nek8 causes left-right asymmetry defects. J. Am. Soc. Nephrol. 24, 100-112. doi: 10.1681/ASN.2012050490

Marion, V., Schlicht, D., Mockel, A., Caillard, S., Imhoff, O., Stoetzel, C., et al. (2011). Bardet-Biedl syndrome highlights the major role of the primary cilium in efficient water reabsorption. Kidney Int. 79, 1013-1025. doi: 10.1038/ki.2010. 538

Masyuk, T. V., Masyuk, A. I., Torres, V. E., Harris, P. C., and Larusso, N. F. (2007). Octreotide inhibits hepatic cystogenesis in a rodent model of polycystic liver disease by reducing Cholangiocyte adenosine. Gastroenterology 132, 11041116. doi: 10.1053/j.gastro.2006.12.039

McCooke, J. K., Appels, R., Barrero, R. A., Ding, A., Ozimek-Kulik, J. E., Bellgard, M. I., et al. (2012). A novel mutation causing nephronophthisis in the lewis polycystic kidney rat localises to a conserved RCC1 domain in Nek8. BMC Genom. 13:393. doi: 10.1186/1471-2164-13-393
McKee, R. A., and Wingert, R. A. (2016). Repopulating decellularized kidney scaffolds: an avenue for ex vivo organ generation. Materials 9:190. doi: 10.3390/ ma9030190

Mihajlovic, M., Hariri, S., Westphal, K. C. G., Janssen, M. J., Oost, M. J., Bongiovanni, L., et al. (2019a). Safety evaluation of conditionally immortalized cells for renal replacement therapy. Oncotarget 10, 5332-5348.

Mihajlovic, M., Wever, K. E., van der Made, T. K., de Vries, R. B. M., Hilbrands, L. B., and Masereeuw, R. (2019b). Are cell-based therapies for kidney disease safe? A systematic review of preclinical evidence. Pharmacol. Ther. 197, 191211. doi: 10.1016/j.pharmthera.2019.01.004

Mochizuki, T., Saijoh, Y., Tsuchiya, K., Shirayoshi, Y., Takai, S., Taya, C., et al. (1998). Cloning of inv, a gene that controls left/right asymmetry and kidney development. Nature 395, 177-181. doi: 10.1038/255243a0

Molinari, E., and Sayer, J. A. (2017). Emerging treatments and personalised medicine for ciliopathies associated with cystic kidney disease. Expert Opin. Orphan. Drugs 5, 785-798. doi: 10.1080/21678707.2017.1372282

Mollet, G., Salomon, R., Gribouval, O., Silbermann, F., Bacq, D., Landthaler, G., et al. (2002). The gene mutated in juvenile nephronophthisis type 4 encodes a novel protein that interacts with nephrocystin. Nat. Genet. 32, 300-305. doi: $10.1038 /$ ng996

Monirujjaman, M., Devassy, J. G., Yamaguchi, T., Sidhu, N., Kugita, M., Gabbs, M., et al. (2017). Distinct oxylipin alterations in diverse models of cystic kidney diseases. Biochim. Biophys. Acta Mol. Cell Biol. Lipids 1862, 1562-1574. doi: 10.1016/j.bbalip.2017.08.005

Morgan, D., Turnpenny, L., Goodship, J., Dai, W., Majumder, K., Matthews, L., et al. (1998). Inversin, a novel gene in the vertebrate left-right axis pathway, is partially deleted in the inv mouse. Nat. Genet. 20, 149-156. doi: 10.1038/2450

Müller, R. U., and Schermer, B. (2019). Hippo signaling-a central player in cystic kidney disease? Pediatr. Nephrol. 35, 1143-1152.

Müller, R. U., and Schermer, B. (2020). Hippo signaling-a central player in cystic kidney disease? Pediatr. Nephrol. 35, 1143-1152. doi: 10.1007/s00467019-04299-3

Mykytyn, K., and Askwith, C. (2017). G-Protein-Coupled receptor signaling in cilia. Cold Spring Harb. Perspect. Biol. 9, 1-16. doi: 10.1101/cshperspect. a028183

Nager, A. R., Goldstein, J. S., Herranz-Pérez, V., Portran, D., Ye, F., GarciaVerdugo, J. M., et al. (2017). An Actin network dispatches ciliary GPCRs into extracellular vesicles to modulate signaling. Cell 168, 252-263.e14. doi: 10.1016/ j.cell.2016.11.036

Nandadasa, S., Kraft, C. M., Wang, L. W., O’Donnell, A., Patel, R., Gee, H. Y., et al. (2019). Secreted metalloproteases ADAMTS9 and ADAMTS20 have a non-canonical role in ciliary vesicle growth during ciliogenesis. Nat. Commun. 10:953. doi: 10.1038/s41467-019-08520-7

Nandadasa, S., Nelson, C. M., and Apte, S. S. (2015). ADAMTS9-mediated extracellular matrix dynamics regulates umbilical cord vascular smooth muscle differentiation and rotation. Cell Rep. 11, 1519-1528. doi: 10.1016/j.celrep.2015. 05.005

Natoli, T. A., Husson, H., Rogers, K. A., Smith, L. A., Wang, B., Budman, Y., et al. (2012). Loss of GM3 synthase gene, but not sphingosine kinase 1, is protective against murine nephronophthisis-related polycystic kidney disease. Hum. Mol. Genet. 21, 3397-3407. doi: 10.1093/hmg/dds172

Natoli, T. A., Modur, V., and Ibraghimov-Beskrovnaya, O. (2020). Glycosphingolipid metabolism and polycystic kidney disease. Cell. Signal. 69:109526. doi: 10.1016/j.cellsig.2020.109526

Natoli, T. A., Smith, L. A., Rogers, K. A., Wang, B., Komarnitsky, S., Budman, Y., et al. (2010). Inhibition of glucosylceramide accumulation results in effective blockade of polycystic kidney disease in mouse models. Nat. Med. 16, 788-792. doi: 10.1038/nm.2171.Inhibition

Nielsen, S., Frøkiær, J., Marples, D., Kwon, T. H., Agre, P., and Knepper, M. A. (2002). Aquaporins in the kidney: from molecules to medicine. Physiol. Rev. 82, 205-244. doi: 10.1152/physrev.00024.2001

Nogueira, A., Pires, M. J., and Oliveira, P. A. (2017). Pathophysiological mechanisms of renal fibrosis: a review of animal models and therapeutic strategies. In Vivo 31, 1-22. doi: 10.21873/invivo.11019

Norris, D. P., and Grimes, D. T. (2012). Mouse models of ciliopathies: the state of the art. DMM Dis. Model. Mech. 5, 299-312. 
Nurnberger, J., Bacallao, R. L., and Phillips, C. L. (2002). Inversin forms a complex with catenins and N-cadherin in polarized epithelial cells. Mol. Biol. Cell 13, 3096-3106. doi: 10.1091/mbc.E02

Oberbauer, R., Schreiner, G. F., Biber, J., Murer, H., and Meyer, T. W. (1996). In vivo suppression of the renal $\mathrm{Na}+/ \mathrm{Pi}$ cotransporter by antisense oligonucleotides. Proc. Natl. Acad. Sci. U.S.A. 93, 4903-4906.

Oey, O., Rao, P., Luciuk, M., Mannix, C., Rogers, N. M., Sagar, P., et al. (2018). Effect of dimethyl fumarate on renal disease progression in a genetic ortholog of nephronophthisis. Exp. Biol. Med. 243, 428-436. doi: 10.1177/ 1535370218759313

Okumura, Y., Sugiyama, N., Tanimura, S., Nishida, M., Hamaoka, K., Kohno, M., et al. (2009). ERK regulates renal cell proliferation and renal cyst expansion in inv mutant mice. Acta Histochem. Cytochem. 42, 39-45. doi: 10.1267/ahc.08040

Olbrich, H., Fliegauf, M., Hoefele, J., Kispert, A., Otto, E., Volz, A., et al. (2003). Mutations in a novel gene, NPHP3, cause adolescent nephronophthisis, tapetoretinal degeneration and hepatic fibrosis. Nat. Genet. 34, 455-459. doi: 10.1038/ ng1216

Omori, S., Hida, M., Fujita, H., Takahashi, H., Tanimura, S., Kohno, M., et al. (2006). Extracellular signal-regulated kinase inhibition slows disease progression in mice with polycystic kidney disease. J. Am. Soc. Nephrol. 17, 1604-1614. doi: 10.1681/ASN.2004090800

Omran, H., Fernandez, C., Jung, M., Haffner, K., Fargier, B., Villaquiran, A., et al. (2000). Identification of a new gene locus for adolescent nephronophthisis, on chromosome 3q22 in a large Venezuelan pedigree. Am. J. Hum. Genet. 66, 118-127. doi: 10.1086/302705

Omran, H., Häffner, K., Burth, S., Fernandez, C., Fargier, B., Villaquiran, A., et al. (2001). Human adolescent nephronophthisis: gene locus synteny with polycystic kidney disease in pcy mice. J. Am. Soc. Nephrol. 12, 107-113.

Oroojalian, F., Charbgoo, F., Hashemi, M., Amani, A., Yazdian-Robati, R., Mokhtarzadeh, A., et al. (2020). Recent advances in nanotechnology-based drug delivery systems for the kidney. J. Control. Release 321, 442-462. doi: 10.1016/j.jconrel.2020.02.027

O’Toole, J. F., Liu, Y., Davis, E. E., Westlake, C. J., Attanasio, M., Otto, E. A., et al. (2010). Individuals with mutations in XPNPEP3, which encodes a mitochondrial protein, develop a nephronophthisis-like nephropathy John. J. Clin. Invest. 120, 791-802. doi: 10.1172/JCI40076.Introduction

O’Toole, J. F., Otto, E. A., Frishberg, Y., and Hildebrandt, F. (2006). Retinitis pigmentosa and renal failure in a patient with mutations in INVS. Nephrol. Dial. Transplant. 21, 1989-1991. doi: 10.1093/ndt/gfl088

Otto, E., Hoefele, J., Ruf, R., Mueller, A. M., Hiller, K. S., Wolf, M. T., et al. (2002). A gene mutated in nephronophthisis and retinitis pigmentosa encodes a novel protein, nephroretinin, conserved in evolution. Am. J. Hum. Genet. 71, 1161-1167. doi: 10.1086/344395

Otto, E. A., Hurd, T. W., Airik, R., Chaki, M., Zhou, W., Stoetzel, C., et al. (2010). Candidate exome capture identifies mutation of SDCCAG8 as the cause of a retinal-renal ciliopathy. Nat. Genet. 42, 840-850. doi: 10.1038/ng.662

Otto, E. A., Loeys, B., Khanna, H., Hellemans, J., Sudbrak, R., Fan, S., et al. (2005). Nephrocystin-5, a ciliary IQ domain protein, is mutated in SeniorLoken syndrome and interacts with RPGR and calmodulin. Nat. Genet. 37, 282-288. doi: 10.1038/ng1520

Otto, E. A., Schermer, B., Obara, T., Toole, J. F. O., Hiller, K. S., Mueller, A. M., et al. (2003). Mutations in INVS encoding inversin cause nephronophthisis type 2, linking renal cystic disease to the function of primary cilia and left-right axis determination. Nat. Genet. 34, 413-420. doi: 10.1038/ng1217.Mutations

Otto, E. A., Trapp, M. L., Schultheiss, U. T., Helou, J., Quarmby, L. M., and Hildebrandt, F. (2008). NEK8 mutations affect ciliary and centrosomal localization and may cause nephronophthisis. J. Am. Soc. Nephrol. 19, 587-592. doi: 10.1681/ASN.2007040490

Pandey, G., Westhoff, J. H., Schaefer, F., and Gehrig, J. (2019). A smart imaging workflow for organ-specific screening in a cystic kidney Zebrafish disease model. Int. J. Mol. Sci. 20:1290. doi: 10.3390/ijms20061290

Parfitt, D. A., Lane, A., Ramsden, C. M., Carr, A. J. F., Munro, P. M., Jovanovic, K., et al. (2016). Identification and Correction of Mechanisms Underlying Inherited Blindness in Human iPSC-Derived Optic Cups. Cell Stem Cell 18, 769-781. doi: 10.1016/j.stem.2016.03.021

Peired, A. J., Mazzinghi, B., De Chiara, L., Guzzi, F., Lasagni, L., Romagnani, P., et al. (2020). Bioengineering strategies for nephrologists: kidney was not built in a day. Expert Opin. Biol. Ther. 20, 467-480. doi: 10.1080/14712598.2020. 1709439

Perrault, I., Saunier, S., Hanein, S., Filhol, E., Bizet, A. A., Collins, F., et al. (2012). Mainzer-saldino syndrome is a ciliopathy caused by IFT140 mutations. Am. J. Hum. Genet. 90, 864-870. doi: 10.1016/j.ajhg.2012.03.006

Phillips, C. L., Miller, K. J., Filson, A. J., Nurnberger, J., Clendenon, J. L., Cook, G. W., et al. (2004). Renal cysts of inv/inv mice resemble early infantile nephronophthisis. J. Am. Soc. Nephrol. 15, 1744-1755.

Phillips, J. K., Hopwood, D., Loxley, R. A., Ghatora, K., Coombes, J. D., Tan, Y. S., et al. (2007). Temporal relationship between renal cyst development, hypertension and cardiac hypertrophy in a new rat model of autosomal recessive polycystic kidney disease. Kidney Blood Press. Res. 30, 129-144. doi: $10.1159 / 000101828$

Phua, S. C., Chiba, S., Suzuki, M., Su, E., Roberson, E. C., Pusapati, G. V., et al. (2017). Dynamic remodeling of membrane composition drives cell cycle through primary cilia excision. Cell 168, 264-279.e15. doi: 10.1016/j.cell.2016. 12.032

Pugacheva, E. N., Jablonski, S. A., Hartman, T. R., Henske, E. P., and Golemis, E. A. (2007). HEF1-dependent aurora a activation induces disassembly of the primary cilium. Cell 129, 1351-1363. doi: 10.1016/j.cell.2007.04.035

Qian, Q., Du, H., King, B. F., Kumar, S., Dean, P. G., Cosio, F. G., et al. (2008). Sirolimus reduces polycystic liver volume in ADPKD patients. J. Am. Soc. Nephrol. 19, 631-638. doi: 10.1681/ASN.2007050626

Qin, J., Lin, Y., Norman, R. X., Ko, H. W., Eggenschwiler, J. T., Anderson, K. V., et al. (2011). Intraflagellar transport protein 122 antagonizes sonic hedgehog signaling and controls ciliary localization of pathway components. Proc. Natl. Acad. Sci. U.S.A. 108, 1456-1461.

Rachel, R. A., Yamamoto, E. A., Dewanjee, M. K., May-Simera, H. L., Sergeev, Y. V., Hackett, A. N., et al. (2015). CEP290 alleles in mice disrupt tissue-specific cilia biogenesis and recapitulate features of syndromic ciliopathies. Hum. Mol. Genet. 24, 3775-3791. doi: 10.1093/hmg/ddv123

Rajagopalan, R., Grochowski, C. M., Gilbert, M. A., Falsey, A. M., Coleman, K., Romero, R., et al. (2015). Compound heterozygous mutations in NEK8 in siblings with end-stage renal disease with hepatic and cardiac anomalies. Am. J. Med. Genet. Part A 170, 750-753. doi: 10.1002/ajmg.a.37512

Ramsbottom, S. A., Miles, C., and Sayer, J. A. (2015). Murine Cep290 phenotypes are modified by genetic backgrounds and provide an impetus for investigating disease modifier alleles. F1000Research 4:590. doi: 10.12688/f1000research. 6959.1

Ramsbottom, S. A., Molinari, E., Srivastava, S., Silberman, F., Henry, C., Alkanderi, S., et al. (2018). Targeted exon skipping of a CEP290 mutation rescues Joubert syndrome phenotypes in vitro and in a murine model. Proc. Natl. Acad. Sci. U.S.A. 115, 12489-12494. doi: 10.1073/pnas.1809432115

Ramsbottom, S. A., Thelwall, P. E., Wood, K. M., Clowry, G. J., Devlin, L. A., Silbermann, F., et al. (2020). Mouse genetics reveals Barttin as a genetic modifier of Joubert syndrome. Proc. Natl. Acad. Sci. U.S.A. 117, 1113-1118. doi: 10.1073/ pnas. 1912602117

Reif, G. A., Yamaguchi, T., Nivens, E., Fujiki, H., Pinto, C. S., and Wallace, D. P. (2011). Tolvaptan inhibits ERK-dependent cell proliferation, $\mathrm{Cl}$ - secretion, and in vitro cyst growth of human ADPKD cells stimulated by vasopressin. Am. J. Physiol. Ren. Physiol. 301, 1005-1013. doi: 10.1152/ajprenal.00243.2011

Reiter, J. F., Leroux, M. R., Francisco, S., Francisco, S., and Columbia, B. (2017). Genes and molecular pathways underpinning ciliopathies. Nat. Rev. Mol. Cell Biol. 18, 533-547. doi: 10.1038/nrm.2017.60.Genes

Riccardi, D., and Valenti, G. (2016). Localization and function of the renal calciumsensing receptor. Nat. Rev. Nephrol. 12, 414-425.

Ronquillo, C. C., Hanke-Gogokhia, C., Revelo, M. P., Frederick, J. M., Jiang, L., and Baehr, W. (2016). Ciliopathy-associated IQCB1/NPHP5 protein is required for mouse photoreceptor outer segment formation. FASEB J. 30, 3400-3412. doi: 10.1096/fj.201600511R

Rosenthal, W., Seibold, A., Antaramian, A., Lonergan, M., Arthus, M.-F., Hendy, G. N., et al. (1992). Molecular identification of the gene responsible for congenital nephrogenic diabetes insipidus. Nature 359, 233-235.

Rossi, A. G., Sawatzky, D. A., Walker, A., Ward, C., Sheldrake, T. A., Riley, N. A., et al. (2006). Cyclin-dependent kinase inhibitors enhance the resolution of inflammation by promoting inflammatory cell apoptosis. Nat. Med. 12, 1056-1064. doi: 10.1038/nm1468 
Ruan, G. X., Barry, E., Yu, D., Lukason, M., Cheng, S. H., and Scaria, A. (2017). CRISPR/Cas9-mediated genome editing as a therapeutic approach for leber congenital amaurosis 10. Mol. Ther. 25, 331-341. doi: 10.1016/j.ymthe.2016.12. 006

Rubin, J. D., Nguyen, T. V., Allen, K. L., Ayasoufi, K., and Barry, M. A. (2019). Comparison of Gene Delivery to the Kidney by adenovirus, adeno-associated virus, and lentiviral vectors after intravenous and direct Kidney Injections. Hum. Gene Ther. 30, 1559-1571. doi: 10.1089/hum.2019.127

Ruggenenti, P., Remuzzi, A., Ondel, P., Fasolini, G., Antiga, L., Ene-Iordache, B., et al. (2005). Safety and efficacy of long-acting somatostatin treatment in autosomal-dominant polycystic kidney disease. Kidney Int. 68, 206-216. doi: 10.1111/j.1523-1755.2005.00395.x

Ruiz-Perez, V. L., Blair, H. J., Rodriguez-andres, M. E., Blanco, M. J., Wilson, A., Liu, Y., et al. (2007). Evc is a positive mediator of Ihh-regulated bone growth that localises at the base of chondrocyte cilia. Development 134, 2903-2912. doi: $10.1242 /$ dev.007542

Saigusa, T., Dang, Y., Mullick, A. E., Yeh, S. T., Zile, M. R., Baicu, C. F., et al. (2016). Suppressing angiotensinogen synthesis attenuates kidney cyst formation in a Pkd1 mouse model. FASEB J. 30, 370-379. doi: 10.1096/fj.15-279299

Salomon, R., Saunier, S., and Niaudet, P. (2009). Nephronophthisis. Pediatr. Nephrol. 24, 2333-2344. doi: 10.1007/s00467-008-0840-z

Sang, L., Miller, J. J., Corbit, K. C., Giles, R. H., Brauer, M. J., Otto, E. A., et al. (2011). Mapping the Nephronophthisis-joubert-meckel-gruber protein network reveals ciliopathy disease genes and pathways. Cell 145, 513-528. doi: 10.1016/j.cell.2011.04.019.Mapping

Sankaran, D., Bankovic-Calic, N., Ogborn, M. R., Crow, G., and Aukema, H. M. (2007). Selective COX-2 inhibition markedly slows disease progression and attenuates altered prostanoid production in Han:SPRD-cy rats with inherited kidney disease. Am. J. Physiol. Ren. Physiol. 293, 821-830. doi: 10.1152/ajprenal. 00257.2006

Sankaran, D., Lu, J., Bankovic-Calic, N., Ogborn, M. R., and Aukema, H. M. (2004). Modulation of renal injury in pcy mice by dietary fat containing n-3 fatty acids depends on the level and type of fat. Lipids 39, 207-214. doi: 10.1007/s11745004-1221-7

Saunier, S., Calado, J., Heilig, R., Silbermann, F., Benessy, F., Morin, G., et al. (1997). A novel gene that encodes a protein with a putative src homology 3 domain is a candidate gene for familial juvenile nephronophthisis. Hum. Mol. Genet. 6, 2317-2323.

Sayer, J. A., Otto, E. A., O’Toole, J. F., Nurnberg, G., Kennedy, M. A., Becker, C., et al. (2006). The centrosomal protein nephrocystin- 6 is mutated in Joubert syndrome and activates transcription factor ATF4. Nat. Genet. 38, 674-681. doi: $10.1038 /$ ng 1786

Schmidts, M., Frank, V., Eisenberger, T., Al Turki, S., Bizet, A. A., Antony, D., et al. (2013). Combined NGS approaches identify mutations in the intraflagellar transport gene IFT140 in skeletal ciliopathies with early progressive kidney disease. Hum. Mutat. 34, 714-724. doi: 10.1126/scitranslmed.3008882. Plasmodium

Schonauer, R., Jin, W., Ertel, A., Nemitz-Kliemchen, M., Panitz, N., Hantmann, E., et al. (2020). Novel nephronophthisis-associated variants reveal functional importance of MAPKBP1 dimerization for centriolar recruitment. Kidney Int. 98, 958-969. doi: 10.1016/j.kint.2020.05.027

Schueler, M., Braun, D. A., Chandrasekar, G., Gee, H. Y., Klasson, T. D., Halbritter, J., et al. (2015). DCDC2 mutations cause a renal-hepatic ciliopathy by disrupting Wnt signaling. Am. J. Hum. Genet. 96, 81-92. doi: 10.1016/j.ajhg. 2014.12.002

Schutgens, F., Rookmaaker, M. B., Margaritis, T., Rios, A., Ammerlaan, C., Jansen, J., et al. (2019). Tubuloids derived from human adult kidney and urine for personalized disease modeling. Nat. Biotechnol. 37, 303-313. doi: 10.1038/ s41587-019-0048-8

Serra, A. L., Poster, D., Kistler, A. D., Krauer, F., Raina, S., Young, J., et al. (2010). Sirolimus and kidney growth in autosomal dominant polycystic kidney disease. N. Engl. J. Med. 363, 820-829. doi: 10.1056/NEJMoa0907419

Shamseldin, H. E., Shaheen, R., Ewida, N., Bubshait, D. K., Alkuraya, H., Almardawi, E., et al. (2020). The morbid genome of ciliopathies: an update. Genet. Med. 22, 1051-1060. doi: 10.1038/s41436-020-0761-1

Shao, W., Yang, J., He, M., Yu, X. Y., Lee, C. H., Yang, Z., et al. (2020). Centrosome anchoring regulates progenitor properties and cortical formation. Nature 580, 106-112. doi: 10.1038/s41586-020-2139-6
Sharma, N., Kosan, Z. A., Stallworth, J. E., Berbari, N. F., and Yoder, B. K. (2011). Soluble levels of cytosolic tubulin regulate ciliary length control. Mol. Biol. Cell 22, 806-816. doi: 10.1091/mbc.E10-03-0269

Sherpa, R. T., Mohieldin, A. M., Pala, R., Wachten, D., Ostrom, R. S., and Nauli, S. M. (2019). Sensory primary cilium is a responsive cAMP microdomain in renal epithelia. Sci. Rep. 9, 1-16. doi: 10.1038/s41598-019-43002-2

Shillingford, J. M., Leamon, C. P., Vlahov, I. R., and Weimbs, T. (2012). Folateconjugated rapamycin slows progression of polycystic kidney disease. J. Am. Soc. Nephrol. 23, 1674-1681. doi: 10.1681/ASN.2012040367

Shillingford, J. M., Murcia, N. S., Larson, C. H., Low, S. H., Hedgepeth, R., Brown, N., et al. (2006). The mTOR pathway is regulated by polycystin-1, and its inhibition reverses renal cystogenesis in polycystic kidney disease. Proc. Natl. Acad. Sci. U.S.A. 103, 5466-5471. doi: 10.1073/pnas.0509694103

Simons, D. L., Boye, S. L., Hauswirth, W. W., and Wu, S. M. (2011). Gene therapy prevents photoreceptor death and preserves retinal function in a Bardet-Biedl syndrome mouse model. Proc. Natl. Acad. Sci. U.S.A. 108, 6276-6281. doi: 10.1073/pnas.1019222108

Simons, M., Gloy, J., Ganner, A., Bullerkotte, A., Bashkurov, M., Kronig, C., et al. (2005). Inversin, the gene product mutated in nephronophthisis type II, functions as a molecular switch between Wnt signaling pathways. Nat. Genet. 37, 537-543. doi: 10.1038/ng1552

Skelding, K. A., Rostas, J. A. P., and Verrills, N. M. (2011). Controlling the cell cycle: the role of calcium/calmodulin-stimulated protein kinases I and II. Cell Cycle 10, 631-639. doi: 10.4161/cc.10.4.14798

Slaats, G. G., Ghosh, A. K., Falke, L. L., Le Corre, S., Shaltiel, I. A., van de Hoek, G., et al. (2014). Nephronophthisis-associated CEP164 regulates cell cycle progression, apoptosis and epithelial-to-mesenchymal transition. PLoS Genet. 10:e1004594. doi: 10.1371/journal.pgen.1004594

Slaats, G. G., and Giles, R. H. (2015). Are renal ciliopathies (replication) stressed out? Trends Cell Biol. 25, 317-319. doi: 10.1016/j.tcb.2015.03.005

Slaats, G. G., Lilien, M. R., and Giles, R. H. (2016). Nephronophthisis: should we target cysts or fibrosis? Pediatr. Nephrol. 31, 545-554. doi: 10.1007/s00467-0153162-y

Slaats, G. G., Saldivar, J. C., Bacal, J., Zeman, M. K., Kile, A. C., Hynes, A. M., et al. (2015). DNA replication stress underlies renal phenotypes in CEP290 associated Joubert syndrome. J. Clin. Invest. 125, 1-10. doi: 10.1172/JCI80657. of

Slanchev, K., Pütz, M., Schmitt, A., Kramer-Zucker, A., and Walz, G. (2011). Nephrocystin-4 is required for pronephric duct-dependent cloaca formation in zebrafish. Hum. Mol. Genet. 20, 3119-3128. doi: 10.1093/hmg/ddr214

Smith, L. A., Bukanov, N. O., Husson, H., Russo, R. J., Barry, T. C., Taylor, A. L., et al. (2006). Development of polycystic kidney disease in juvenile cystic kidney mice: insights into pathogenesis, ciliary abnormalities, and common features with human disease. J. Am. Soc. Nephrol. 17, 2821-2831. doi: 10.1681/ASN. 2006020136

Smith, U. M., Consugar, M., Tee, L. J., McKee, B. M., Maina, E. N., Whelan, S., et al. (2006). The transmembrane protein meckelin (MKS3) is mutated in Meckel-Gruber syndrome and the wpk rat. Nat. Genet. 38, 191-196. doi: $10.1038 / \mathrm{ng} 1713$

Snoek, R., van Setten, J., Keating, B. J., Israni, A. K., Jacobson, P. A., Oetting, W. S., et al. (2018). NPHP1 (Nephrocystin-1) gene deletions cause adult-onset ESRD. J. Am. Soc. Nephrol. 29, 1772-1779. doi: 10.1681/asn.2017111200

Sohara, E., Luo, Y., Zhang, J., Manning, D. K., Beier, D. R., and Zhou, J. (2008). Nek8 regulates the expression and localization of polycystin-1 and polycystin- 2 . J. Am. Soc. Nephrol. 19, 469-476. doi: 10.1681/ASN.2006090985

Song, Z., Zhang, X., Jia, S., Yelick, P. C., and Zhao, C. (2016). Zebrafish as a model for human ciliopathies. J. Genet. Genom. 43, 107-120. doi: 10.1016/j.jgg.2016. 02.001

Spasic, M., and Jacobs, C. R. (2017). Primary cilia: cell and molecular mechanosensors directing whole tissue function. Semin. Cell Dev. Biol. 71, 42-52. doi: 10.1016/j.semcdb.2017.08.036.Primary

Srivastava, S., Molinari, E., Raman, S., and Sayer, J. A. (2018). Many genesone disease? Genetics of nephronophthisis (NPHP) and NPHP-associated disorders. Front. Pediatr. 5:287. doi: 10.3389/fped.2017.00287

Srivastava, S., Ramsbottom, S. A., Molinari, E., Alkanderi, S., Filby, A., White, K., et al. (2017). A human patient-derived cellular model of Joubert syndrome reveals ciliary defects which can be rescued with targeted therapies. Hum. Mol. Genet. 26, 4657-4667. doi: 10.1093/hmg/ddx347 
Steichen, C., Giraud, S., and Hauet, T. (2020). Combining kidney Organoids and genome editing technologies for a better understanding of physiopathological mechanisms of renal diseases: state of the art. Front. Med. 7:10. doi: 10.3389/ fmed.2020.00010

Stokman, M. F., Van der Zwaag, B., Van de Kar, N. C. A. J., Van Haelst, M. M., Van Eerde, A. M., Van der Heijden, J. W., et al. (2018). Clinical and genetic analyses of a Dutch cohort of 40 patients with a nephronophthisis-related ciliopathy. Pediatr. Nephrol. 33, 1701-1712. doi: 10.1007/s00467-018-3958-7

Strong, A., Muneeruddin, S., Parrish, R., Lui, D., and Conley, S. B. (2018). Isosorbide dinitrate in nephronophthisis treatment. Am. J. Med. Genet. Part A 176, 1023-1026. doi: 10.1002/ajmg.a.38650

Sugiyama, N., Kohno, M., and Yokoyama, T. (2012). Inhibition of the p38 MAPK pathway ameliorates renal fibrosis in an NPHP2 mouse model. Nephrol. Dial. Transplant. 27, 1351-1358. doi: 10.1093/ndt/gfr550

Sugiyama, N., Tsukiyama, T., Yamaguchi, T. P., and Yokoyama, T. (2011). The canonical Wnt signaling pathway is not involved in renal cyst development in the kidneys of inv mutant mice. Kidney Int. 79, 957-965.

Sussman, C. R., Wang, X., Chebib, F. T., and Torres, V. E. (2020). Modulation of polycystic kidney disease by G-protein coupled receptors and cyclic AMP signaling. Cell. Signal. 72:109649. doi: 10.1016/j.cellsig.2020.109649

Suzuki, A., Ogata, K., Yoshioka, H., Shim, J., Wassif, C. A., Porter, F. D., et al. (2020). Disruption of Dhcr7 and Insig1/2 in cholesterol metabolism causes defects in bone formation and homeostasis through primary cilium formation. Bone Res. 8:1. doi: 10.1038/s41413-019-0078-3

Szeto, S. G., Narimatsu, M., Lu, M., He, X., Sidiqi, A. M., Tolosa, M. F., et al. (2016). YAP/TAZ are mechanoregulators of TGF-b-smad signaling and renal fibrogenesis. J. Am. Soc. Nephrol. 27, 3117-3128. doi: 10.1681/ASN.2015050499

Takahashi, H., Calvet, J. P., Dittemore-Hoover, D., Yoshida, K., Grantham, J. J., and Gattone, V. H. (1991). A hereditary model of slowly progressive polycystic kidney disease in the mouse. J. Am. Soc. Nephrol. 1, 980-989.

Takasato, M., Er, P. X., Chiu, H. S., Maier, B., Baillie, G. J., Ferguson, C., et al. (2015). Kidney organoids from human iPS cells contain multiple lineages and model human nephrogenesis. Nature 526, 564-569.

Tan, R. J., Zhou, D., and Liu, Y. (2016). Signaling crosstalk between tubular epithelial cells and interstitial fibroblasts after kidney injury. Kidney Dis. 2, 136-144. doi: 10.1159/000446336

Tao, Y., Kim, J., Schrier, R. W., and Edelstein, C. L. (2005). Rapamycin markedly slows disease progression in a rat model of polycystic kidney disease. J. Am. Soc. Nephrol. 16, 46-51. doi: 10.1681/ASN.2004080660

Taskiran, E. Z., Korkmaz, E., Gucer, S., Kosukcu, C., Kaymaz, F., Koyunlar, C., et al. (2014). Mutations in ANKS6 cause a nephronophthisis-like phenotype with ESRD. J. Am. Soc. Nephrol. 25, 1653-1661. doi: 10.1681/ASN.2013060646

Tayfur, A. C., Besbas, N., Bilginer, Y., Ozaltin, F., Duzova, A., Bakkaloglu, M., et al. (2011). Follow-up of patients with juvenile nephronophthisis after renal transplantation: a single center experience. Transplant. Proc. 43, 847-849.

Tobin, J. L., and Beales, P. L. (2008). Restoration of renal function in zebrafish models of ciliopathies. Pediatr. Nephrol. 23, 2095-2099. doi: 10.1007/s00467008-0898-7

Torres, V. E., Chapman, A. B., Devuyst, O., Gansevoort, R. T., Grantham, J. J., Higashihara, E., et al. (2012). Tolvaptan in patients with autosomal dominant polycystic kidney disease. N. Engl. J. Med. 367, 2407-2418. doi: 10.1056/ NEJMoa1205511

Torres, V. E., Chapman, A. B., Devuyst, O., Gansevoort, R. T., Perrone, R. D., Dandurand, A., et al. (2018). Multicenter, open-label, extension trial to evaluate the long-term efficacy and safety of early versus delayed treatment with tolvaptan in autosomal dominant polycystic kidney disease: the TEMPO 4:4 Trial. Nephrol. Dial. Transplant. 33, 477-489. doi: 10.1093/ndt/gfx043

Torres, V. E., Chapman, A. B., Devuyst, O., Gansevoort, R. T., Perrone, R. D., Koch, G., et al. (2017). Tolvaptan in later-stage autosomal dominant polycystic kidney disease. N. Engl. J. Med. 377, 1930-1942. doi: 10.1056/NEJMoa1710030

Torres, V. E., Wang, X., Qian, Q., Somlo, S., Harris, P. C., and Ii, V. H. G. (2004). Effective treatment of an orthologous model of autosomal dominant polycystic kidney disease. Nat. Med. 10, 363-364. doi: 10.1038/nm1004

Tory, K., Lacoste, T., Burglen, L., Morinière, V., Boddaert, N., Macher, M. A., et al. (2007). High NPHP1 and NPHP6 mutation rate in patients with Joubert syndrome and nephronophthisis: potential epistatic effect of NPHP6 and AHI1 mutations in patients with NPHP1 mutations. J. Am. Soc. Nephrol. 18, 15661575. doi: 10.1681/ASN.2006101164
Tory, K., Rousset-Rouvière, C., Gubler, M. C., Morinière, V., Pawtowski, A., Becker, C., et al. (2009). Mutations of NPHP2 and NPHP3 in infantile nephronophthisis. Kidney Int. 75, 839-847. doi: 10.1038/ki.2008.662

Toya, M., and Takeichi, M. (2016). Organization of non-centrosomal microtubules in epithelial cells. Cell Struct. Funct. 41, 127-135. doi: 10.1247/csf. 16015

Tran, P. V., Haycraft, C. J., Besschetnova, T. Y., Turbe-Doan, A., Scottmann, R. W., Herron, B. J., et al. (2008). THM1 negatively modulates mouse sonic hedgehog signal transduction and affects retrograde intraflagellar transport in cilia. Nat. Genet. 40, 403-410.

Tran, P. V., Talbott, G. C., Turbe-Doan, A., Jacobs, D. T., Schonfeld, M. P., Silva, L. M., et al. (2014). Downregulating hedgehog signaling reduces renal cystogenic potential of mouse models. J. Am. Soc. Nephrol. 25, 2201-2212. doi: 10.1681/ASN.2013070735

Tsai, I. C., Adams, K. A., Tzeng, J. A., Shennib, O., Tan, P. L., and Katsanis, N. (2019). Genome-wide suppressor screen identifies USP35/USP38 as therapeutic candidates for ciliopathies. JCI Insight 4:e130516. doi: 10.1172/jci.insight. 130516

Uchimura, K., Wu, H., Yoshimura, Y., and Humphreys, B. D. (2020). Human pluripotent stem cell-derived kidney organoids with improved collecting duct maturation and injury modeling. Cell Rep. 33:108514. doi: 10.1016/j.celrep. 2020.108514

Valente, E. M., Silhavy, J. L., Brancati, F., Barrano, G., Krishnaswami, S. R., Castori, M., et al. (2006). Mutations in CEP290, which encodes a centrosomal protein, cause pleiotropic forms of Joubert syndrome. Nat. Genet. 38, 623-625.

Van den Ouweland, A. M., Dreesen, J. C., Verdijk, M., Knoers, N. V., Monnens, L. A., Rocchi, M., et al. (1992). Mutations in the vasopressin type 2 receptor gene (AVPR2) associated with nephrogenic diabetes insipidus. Nat. Genet. 2, 99-102.

Viau, A., Bienaimé, F., Lukas, K., Todkar, A. P., Knoll, M., Yakulov, T. A., et al. (2018). Cilia-localized LKB 1 regulates chemokine signaling, macrophage recruitment, and tissue homeostasis in the kidney. EMBO J. 37, 1-21. doi: 10.15252/embj.201798615

Vierkotten, J., Dildrop, R., Peters, T., Wang, B., and Rüther, U. (2007). Ftm is a novel basal body protein of cilia involved in Shh signalling. Development 134, 2569-2577. doi: 10.1242/dev.003715

Wang, J., and Barr, M. M. (2016). Ciliary extracellular vesicles: Txt msg orgnlls. Cell. Mol. Neurobiol. 36, 449-457. doi: 10.1016/j.gde.2016.03. 011

Wang, K., Li, L., Zhang, Y., and Gao, D. (2020). Crosstalk between signaling pathways and DNA damage response. Genome Instab. Dis. 1, 81-91. doi: 10. 1007/s42764-019-00005-7

Wang, Y., Yin, X., Rosen, G., Gabel, L., Guadiana, S. M., Sarkisian, M. R., et al. (2011). Dcdc2 knockout mice display exacerbated developmental disruptions following knockdown of doublecortin. Neurosci. 190, 398-408. doi: 10.1016/j. neuroscience.2011.06.010

Wang, Y., Zhou, C. J., and Liu, Y. (2018). Wnt signaling in kidney development and disease. Prog. Mol. Biol. Transl. Sci. 153, 181-207. doi: 10.1016/bs.pmbts. 2017.11.019

Watanabe, Y., Fujinaga, S., Sakuraya, K., Morisada, N., Nozu, K., and Iijima, K. (2019). Rapidly progressive nephronophthisis in a 2-year-old boy with a homozygous SDCCAG8 mutation. Tohoku J. Exp. Med. 249, 29-32. doi: 10. $1620 /$ tjem. 249.29

Waters, A. M., and Beales, P. L. (2011). Ciliopathies: an expanding disease spectrum. Pediatr. Nephrol. 26, 1039-1056. doi: 10.1007/s00467-0101731-7

Wheway, G., Nazlamova, L., and Hancock, J. T. (2018). Signaling through the primary cilium. Front. Cell Dev. Biol. 6:8. doi: 10.3389/fcell.2018.00008

Wiegering, A., Dildrop, R., Vesque, C., Schneider-Maunoury, S., and Gerhardt, C. (2021). Rpgrip1l controls ciliary gating by ensuring the proper amount of Cep290 at the vertebrate transition zone. Mol Biol Cell 2, 675-689. doi: 10.1101/ 2020.02.10.942300

Wiegering, A., Rüther, U., and Gerhardt, C. (2019). The role of primary cilia in the crosstalk between the Ubiquitin-proteasome system and autophagy. Cells 8:241. doi: $10.3390 /$ cells 8030241

Wolf, M. T., Saunier, S., O’Toole, J. F., Wanner, N., Groshong, T., Attanasio, M., et al. (2007). Mutational analysis of the RPGRIP1L gene in patients with Joubert 
syndrome and nephronophthisis. Kidney Int. 72, 1520-1526. doi: 10.1038/sj.ki. 5002630

Won, J., de Evsikova, C. M., Smith, R. S., Hicks, W. L., Edwards, M. M., Longo-Guess, C., et al. (2011). NPHP4 is necessary for normal photoreceptor ribbon synapse maintenance and outer segment formation, and for sperm development. Hum. Mol. Genet. 20, 482-496. doi: 10.1093/hmg/ddq494

Wood, C. R., Huang, K., Diener, D. R., and Rosenbaum, J. L. (2013). The cilium secretes bioactive ectosomes. Curr. Biol. 23, 906-911. doi: 10.1016/j.cub.2013. 04.019

Wu, M., Gu, J., Mei, S., Xu, D., Jing, Y., Yao, Q., et al. (2016). Resveratrol delays polycystic kidney disease progression through attenuation of nuclear factor $\mathrm{\kappa b}$ induced inflammation. Nephrol. Dial. Transplant. 31, 1826-1834. doi: 10.1093/ ndt/gfw058

Xu, C., Wang, L., Zhang, Y., Li, W., Li, J., Wang, Y., et al. (2020). Tubule-Specific Mst1/2 Deficiency Induces CKD via YAP and Non-YAP Mechanisms. J Am Soc Nephrol 31, 946-961. doi: 10.1681/ASN.2019101052

Yamaguchi, T., Devassy, J. G., Gabbs, M., Ravandi, A., Nagao, S., and Aukema, H. M. (2015). Dietary flax oil rich in $\alpha$-linolenic acid reduces renal disease and oxylipin abnormalities, including formation of docosahexaenoic acid derived oxylipins in the CD1-pcy/pcy mouse model of nephronophthisis. Prostaglandins Leukot. Essent. Fat. Acids 94, 83-89. doi: 10.1016/j.plefa.2014. 11.009

Yamaguchi, T., Lysecki, C., Reid, A., Nagao, S., and Aukema, H. M. (2014). Renal cyclooxygenase products are higher and lipoxygenase products are lower in early disease in the pcy mouse model of adolescent nephronophthisis. Lipids 49, 39-47. doi: 10.1007/s11745-013-3859-2

Yamaguchi, T., Nagao, S., Wallace, D. P., Belibi, F. A., Cowley, B. D., Pelling, J. C., et al. (2003). Cyclic AMP activates B-Raf and ERK in cyst epithelial cells from autosomal-dominant polycystic kidneys. Kidney Int. 63, 1983-1994. doi: 10.1046/j.1523-1755.2003.00023.x

Yamaguchi, T., Pelling, J. C., Ramaswamy, N. T., Eppler, J. W., Wallace, D. P., Nagao, S., et al. (2000). cAMP stimulates the in vitro proliferation of renal cyst epithelial cells by activating the extracellular signal-regulated kinase pathway. Kidney Int. 57, 1460-1471. doi: 10.1046/j.1523-1755.2000.00991.x

Ye, H., Wang, X., Constans, M. M., Sussman, C. R., Chebib, F. T., Irazabal, M. V., et al. (2017). The regulatory $1 \alpha$ subunit of protein kinase a modulates renal cystogenesis. Am. J. Physiol. Ren. Physiol. 313, F677-F686. doi: 10.1152/ ajprenal.00119.2017

Ye, M., and Grantham, J. J. (1993). The secretion of fluid by renal cysts from patients with autosomal dominant polycystic kidney disease. New Engl. J. Med. $329,310-313$.

Yokoyama, T., Copeland, N. G., Jenkins, N. A., Charles, A., Elder, F. F. B., Overbeek, P. A., et al. (1993). Reversal of Left-right asymmetry: a situs inversus mutation. Science 260, 679-682.
Yoshina, S., Sakaki, K., Yonezumi-Hayashi, A., Gengyo-Ando, K., Inoue, H., Iino, Y., et al. (2012). Identification of a novel ADAMTS9/GON-1 function for protein transport from the ER to the Golgi. Mol. Biol. Cell 23, 1728-1741. doi: 10.1091/mbc.E11-10-0857

Yousef Yengej, F. A., Jansen, J., Rookmaaker, M. B., Verhaar, M. C., and Clevers, H. (2020). Kidney Organoids and Tubuloids. Cells 9, 1-20. doi: 10.3390/ cells 9061326

Zafar, I., Tao, Y., Falk, S., McFann, K., Schrier, R. W., and Edelstein, C. L. (2007). Effect of statin and angiotensin-converting enzyme inhibition on structural and hemodynamic alterations in autosomal dominant polycystic kidney disease model. Am. J. Physiol. Ren. Physiol. 293, 854-859. doi: 10.1152/ajprenal.00059. 2007

Zhang, J. Q. J., Burgess, J., Stepanova, D., Saravanabavan, S., Wong, A. T. Y., Kaldis, P., et al. (2020). Role of cyclin-dependent kinase 2 in the progression of mouse juvenile cystic kidney disease. Lab. Investig. 100, 696-711. doi: 10.1038/s41374019-0360-4

Zhang, J. Q. J., Saravanabavan, S., Munt, A., Wong, A. T. Y., Harris, D. C., Harris, P. C., et al. (2019). The role of DNA damage as a therapeutic target in autosomal dominant polycystic kidney. Expert Rev. Mol. Med. 21:e6.

Zhang, P., Kiseleva, A. A., Korobeynikov, V., Liu, H., and Liebau, M. C. (2019). Microscopy-based automated live cell screening for small molecules that affect ciliation. Front. Genet. 10:75. doi: 10.3389/fgene.2019.00075

Zhou, W., Otto, E. A., Cluckey, A., Airik, R., Hurd, T. W., Chaki, M., et al. (2012). FAN1 mutations cause karyomegalic interstitial nephritis, linking chronic kidney failure to defective DNA damage repair. Nat. Genet. 44, 910-917.

Zhu, P., Qiu, Q., Harris, P. C., Xu, X., and Lin, X. (2021). mtor Haploinsufficiency ameliorates renal cysts and cilia abnormality in adult Zebrafish tmem67 mutants. J Am Soc Nephrol 2021:ASN.2020070991.

Zullo, A., Iaconis, D., Barra, A., Cantone, A., Messaddeq, N., Capasso, G., et al. (2010). Kidney-specific inactivation of Ofd1 leads to renal cystic disease associated with upregulation of the mTOR pathway. Hum. Mol. Genet. 19, 2792-2803. doi: $10.1093 / \mathrm{hmg} / \mathrm{ddq} 180$

Conflict of Interest: The authors declare that the research was conducted in the absence of any commercial or financial relationships that could be construed as a potential conflict of interest.

Copyright (c) 2021 Stokman, Saunier and Benmerah. This is an open-access article distributed under the terms of the Creative Commons Attribution License (CC BY). The use, distribution or reproduction in other forums is permitted, provided the original author(s) and the copyright owner(s) are credited and that the original publication in this journal is cited, in accordance with accepted academic practice. No use, distribution or reproduction is permitted which does not comply with these terms. 